\title{
Development of an Optical Disk System for the Automated Retrieval of EASEAR Records
}

\section{Natalie Willman}

U.S. DEPARTMENT OF COMMERCE National Institute of Standards and Technology Advanced Systems Division Computer Systems Laboratory Gaithorsburg, MD 20899

Sponsored by:

Social Security Administration Baltimore, Maryland 



\section{Development of an Optical \\ Disk System for the Automated Retrieval of EASEAR Records}

Natalie Willman

U.S. DEPARTMENT OF COMMERCE National Institute of Standards and Technology

Advanced Sjystems Division Computer Systems Laboratory Gaithersburg, MD 20899

Sponsored by:

Social Security Administration Baltimore, Maryland

August 1991

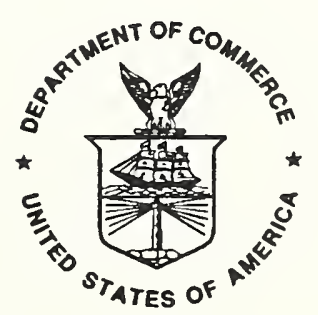

U.S. DEPARTMENT OF COMMERCE Robert A. Mosbacher, Secretary NATIONAL INSTIUUTE OF STANDARDS AND TECHNOLOGY

John W. Lyons, Director 



\section{Acknowledgments}

Many people, in addition to the author of this report, have contributed to the program outlined in this publication, and the author is most appreciative of that assistance. Special thanks are due to the following individuals from NIST: Dana Grubb (Manager, Data Storage Group), Fernando Podio, Mark Williamson, Lloyd Gilmore and Diane Honeycutt.

The support and information provided by the project team at SSA are also appreciated. Special thanks are due to the following individuals from SSA: Malcolm Ewell, Ed Bulson, Marty Mahon, Joan Piasecki, Janice Whelchel, Ann Dempsey, Charlie Fuller, Perry Briscoe, Michael Macer and Michael Banks. 



\section{Table of Contents}

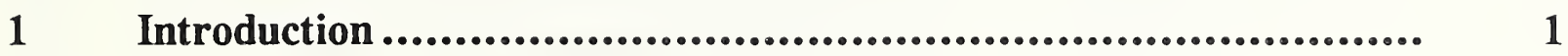

$2 \quad$ Pilot System Components $. . . \ldots \ldots \ldots \ldots \ldots \ldots \ldots \ldots \ldots \ldots \ldots \ldots \ldots \ldots \ldots \ldots \ldots \ldots \ldots . \ldots \ldots$

2.1 Hardware Components......................................... 3

2.2 Software Components........................................... 4

$3 \quad$ Pilot System Design ...................................................... 5

3.1 Operating System ............................................. 5

3.2 Mass Storage Devices and Integration.......................... 6

3.2.1 Mass Storage Media and Drives ......................... 6

3.2.2 Device Integration Methods ............................. 6

3.2.2.1 Small Computer Systems Interface ......... 6

3.2.2.2 Common Access Method................... 7

3.2.3 Open Reel Tape Drive ................................. 7

3.2.4 Optical Disk Library System ............................ 7

4 In-House Software Design $\quad \ldots \ldots \ldots \ldots \ldots \ldots \ldots \ldots \ldots \ldots \ldots \ldots \ldots \ldots \ldots \ldots \ldots \ldots . \ldots \ldots$

4.1 Overview of the EASEAR User Interface........................ 10

4.1.1 General Screen Characteristics............................. 10

4.1 .2 The Query Screen........................................ 11

4.1.3 The Browse Screen ..................................... 12

4.1.4 The Detail Screen............................................. 13

4.1.5 The Error Screens ...................................... 13

4.1.6 Management Information .............................. 13 
4.2 User Interface Software Organization

4.2.1 Software Data Structures ............................... 14

4.2.1.1 The Query Parameters...................... 14

4.2.1.2 The Browse Data ........................... 14

4.2.1.3 The Full Record Data....................... 15

4.2.1.4 The Informix Host Variables ............... 15

4.2.1.5 The Request Message...................... 15

4.2.1.6 The Acknowledge Message .................. 16

4.2.1.7 The Management Information.............. 16

4.2.2 High Level Software Organization ...................... 17

4.2.2.1 Initialization and Control of the

User Interface Environment.............. 17

4.2.2.2 Entry of the User Query .................. 18

4.2.2.3 Selection of the Matches to the Query ...... 18

4.2.2.4 Display and Viewing of Browse Fields ..... 19

4.2.2.5 Display of Detailed Record Information.... 19

4.3 Overview of the Cartridge Handler Device Driver ................ 19

4.3.1 The Scheduler Algorithm .................................. 20

4.3.2 The Control Algorithm................................ 20

4.4 Cartridge Handler Device Driver Software Organization.......... 21

4.4.1 Software Data Structures .............................. 21

4.4.1.1 The Message Queues ...................... 21

4.4.1.2 The Request Message...................... 21

4.4.1.3 The Acknowledge Message ................. 22

4.4.1.4 The Drive Status Information ............... 22

4.4.1.5 The Command Descriptor Block............ 22

4.4.1.6 The SDLP Command Information.......... 23 
4.4.2 High Level Software Organization ...................... 23

4.4.2.1 Initialization of the Data Structures......... 24

4.4.2.2 Receipt of a Request ...................... 24

4.4.2.3 Processing of a Request ................... 24

5 Conversion and Handling of SSA Data................................ 27

5.1 Basic Procedures............................................ 28

5.1.1 Inserting a Disk into a Library Drive ..................... 28

5.1 .2 Ejecting a Disk from a Library Drive ..................... 28

5.1.3 Logging into an Account on the UNIX System ............ 29

5.1.4 Gaining System Administrator Privileges ................. 29

5.1.5 Formatting an Optical Disk ............................... 29

5.1.6 Placing a File System on an Optical Disk................. 29

5.1 .7 Mounting a File System................................... $\quad 30$

5.1 .8 Unmounting a File System ............................... 30

5.1 .9 Logging out of an Account ............................... 30

5.2 Conversion from Tape to Optical Disk.......................... 31

5.2.1 System Procedures....................................... 31

$5.3 \quad$ Data Analysis............................................. $\quad 32$

5.3.1 System Procedures....................................... 32

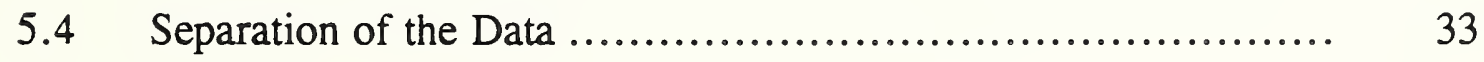

5.4.1 System Procedures....................................... 33

5.5 Conversion of Data to Informix Database Table Format .......... 34

5.5.1 System Procedures...................................... 34

5.6 Compression of the Data ...................................... 35

5.6.1 System Procedures..................................... 35 
A.1 Entering the System ............................................ 39

A.2 The Query Screen ............................................. 40

A.3 The Browse Screen ............................................. 42

A.4 The Detail Screen.............................................. 43

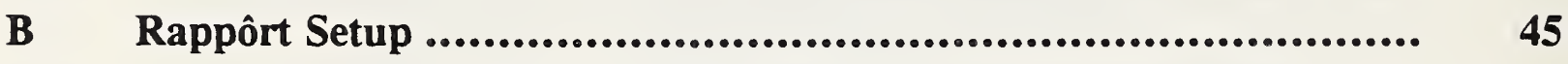

B. 1 Terminal Parameters .............................................. 45

B.2 File Transfer Parameters ........................................ 46

B.3 Screen Colors ................................................... 46

C System Error Messages ............................................... 47

C.1 User Interface Error Messages................................... 47

C.2 Cartridge Handler Device Driver Error Messages................ 48

D Listing of the Code.................................................. 51 


\section{Table of Figures}

Figure 1 System Components .......................................... 3

Figure 2 System Software Flow of Data .................................. 9

Figure 3 Query Screen ................................................ 11

Figure $4 \quad$ Browse Screen ............................................ 12

Figure 5 Detail Screen .................................................. 13

Figure 6 Error Screen ................................................ 13

Figure $7 \quad$ User Interface Software Control Flow............................ 17

Figure $8 \quad$ Cartridge Handler Device Driver Software Control Flow......... 23

Figure 9 Data Conversion Process .................................... 28

Figure $10 \quad$ IRD File................................................... 32

Figure 11 Index File ................................................. 33

Figure A1 Login Screen .............................................. 39

Figure A2 Query Screen ............................................... 40

Figure A3 Browse Screen ............................................. 42

Figure A4 Detail Screen ............................................ 43 



\section{Introduction}

The Social Security Administration (SSA) maintains records of the yearly wages earned by every person in the United States. Each year, approximately 2.5 gigabytes of data are collected on self-employed wage earners and stored in the EASEAR file. Another 47 gigabytes of data are collected on other wage earners and stored in the EAMATE file. The records are currently stored on over 110,000 rolls of microfilm. It takes over 400 scouts to retrieve information needed by the professional staff. Often, the needed roll of film is missing from the file due to being currently in use, misfiled, or misplaced. In addition, the information obtained from the microfilm is not always the most current, and decisions are sometimes based on obsolete data. Advances in peripheral mass storage technology during the 1980s (e.g. magneto-optic recording) now allow for alternative approaches to data storage and retrieval. The agency believes that an automated retrieval system would provide a more accurate, timely, and cost effective means of retrieving the information.

The focus of this document is the evolution and design of the EASEAR pilot application. The full pilot consists of three phases. Phase One of the project consists of the development of an automated retrieval pilot system for two years of the EASEAR records. In Phase Two of the pilot system, one year of the EAMATE records will be added to the pilot system, and in Phase three, a provision will be made for the connection of the pilot system to the SSA mainframe. The goals of the project are as follows: to demonstrate the feasibility of a full scale automated records retrieval system, to study the impact of an automated system on the users, and to gain insight into the factors which may impact the design of a full scale system.

This pilot system, both hardware and software, is intended solely for use in a pilot environment. The NIST developed software, while being tested to the best of our abilities, is not guaranteed to be error free. The pilot system design was intended to minimize cost while placing maximum emphasis on establishing the feasibility of an automated system and studying the factors that would impact the design of a full scale system. The pilot system design used does not imply any recommendations for a full scale system design. Any products named are for descriptive purposes and do not imply a recommendation for a final implementation. 


\section{Pilot system Components}

The EASEAR pilot system is an integration of NIST developed software and commercially available software and hardware. In this way, the pilot system could be tailored to focus on the areas that were of interest to SSA and which could influence a later implementation of a full scale system. The following section discusses the hardware and software components of the pilot system.

\subsection{Hardware Components}

The EASEAR pilot system file server consists of a Compaq 386/20 with a 200 MB hard drive and $12 \mathrm{MB}$ of RAM. The file server is daisy chained, via a Western Digital 7000 FASST-2 SCSI host bus adapter and external SCSI cables, to a Hewlett-Packard $88780 \mathrm{~B} 12.7 \mathrm{~mm}(1 / 2 \mathrm{in})$ open reel tape drive and a Hewlett-Packard C1710A rack mountable optical disk library with two $130 \mathrm{~mm}\left(5 \frac{1}{4} \mathrm{in}\right)$ magneto-optic drives and 32 cartridge slots. The file server also contains an Arnet Smartport-8 multiport adapter board connected to four 19200 baud modems. The power to the file server and its peripherals is regulated by a Sola MCR series $2.0 \mathrm{kVA}$ surge suppressor/voltage regulator.

The workstations in the pilot employ Intel 80286 or 80386 microprocessors in a 100\% IBM compatible AT with color monitors and 101 keyboards. The workstations communicate with the file sever using 19200 baud modems (see Figure 1).

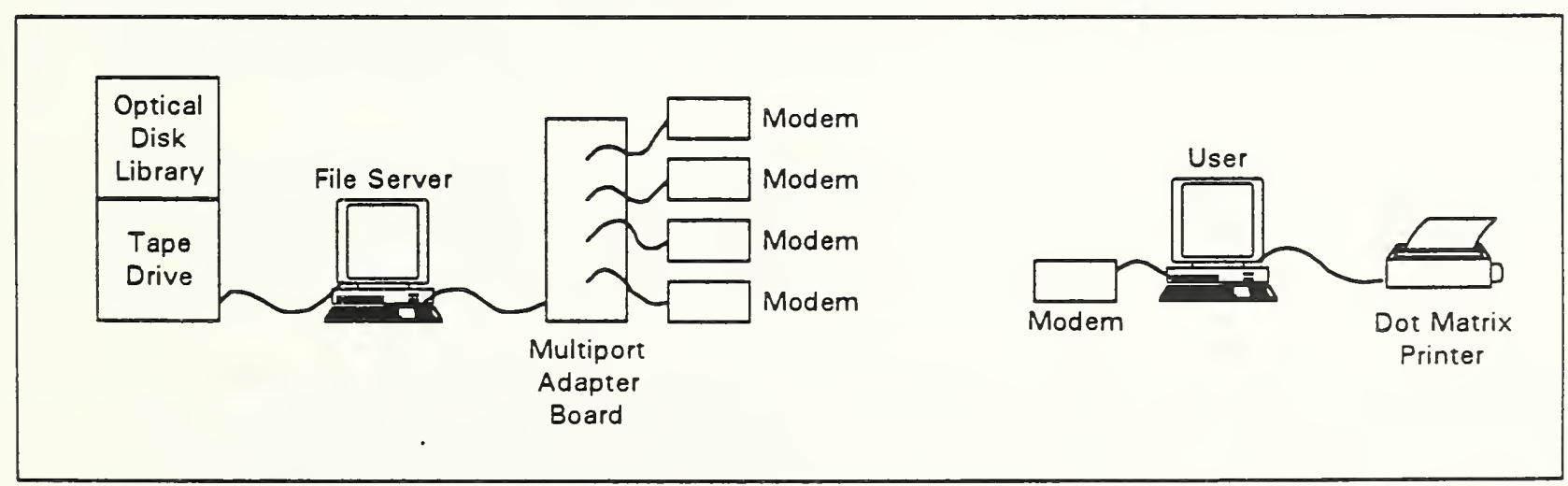

Figure 1. System Components 


\subsection{Software Components}

The operating system for the file server is Interactive UNIX System V Release 3.2, Version. 2.2. With the exception of the optical disk library cartridge handler, the file server's peripherals are controlled by Columbia Data Products software drivers and UNIX utilities. NIST developed a software driver and scheduling algorithm to handle the requests for optical disk cartridges and the movement of the optical disk library cartridge handler. In addition, NIST developed an EASEAR user interface to allow the user to query for and to browse through the EASEAR record data. The Informix ESQL/C embedded SQL database engine is used to manage the EASEAR record data.

The operating system for the workstations is DOS 3.1 or above. Rappôrt, a DOS/UNIX bridge developed by Tundra Software, is used to communicate with the UNIX file server. 


\section{Pilot System Design}

In order to provide SSA with the most feedback on the problems involved in the development of an automated records retrieval system, the design of the pilot system reflects a great effort to remain vendor-independent, while still consisting largely of "off-the-shelf" products. In the choice of components, NIST attempted to closely follow the existing and emerging standards in the following issues: removable optical disk media, Small Computer Systems Interface (SCSI) devices and programming interfaces, Structured Query Language (SQL) databases, and operating systems (see Reference Section):

The main goals of the pilot system were to demonstrate the feasibility of an automated retrieval system and to study the impact of the system on the users. Considering these goals, it was determined that the most crucial areas in the design of the pilot system were the operating system, the integration of a mass storage capability, the indexing methods used to retrieve records, and the user interface. This section of the document concentrates on the operating system and mass storage devices, while issues concerning the user interface and indexing methods are discussed in the software section of this document.

\subsection{Operating System}

The main criteria for the file server operating system was that it provided multiuser access, a POSIX-like interface, and supported removable optical disk media. With these criteria in mind, the following operating systems were considered: OS/2 extended edition, Novell Netware and UNIX. Based on the factors outlined below, NIST chose UNIX for the file server operating system.

While OS/2 would merge well with SSA's existing IBM mainframe hardware, it is still a fairly new operating system. Along with several other minor flaws in its current version, OS/2 does not currently provide adequate support for SCSI optical disk drives.

While Novell Netware has the most dominant networking operating system on the market, at this time it does not support removable optical media. According to Novell's development engineers, Novell will not guarantee that their operating system will work with removable optical media, and actually strongly recommended that Novell Netware and removable optical media not be used together. 
UNIX had many advantages over the other operating systems. It is a well established operating system with a large market share, considerable power and flexibility, and an interface which supports the spirit of POSIX. UNIX supports optical media with no compromise to its efficiency, and was repeatedly recommended by indistry developers for use with this type of media, drives and libraries.

Implementations of the UNIX operating system available for Intel 80386 microprocessor based systems were considered. NIST chose to use Interactive Systems Corporation's (ISC) Interactive UNIX System V Release 3.2.

\subsection{Mass Storage Devices and Integration}

The integration of a mass storage capability into the pilot system involved the consideration of a large number of issues. The following are the major areas that were researched: mass storage media, mass storage device integration methods, open reel tape drives, and automated library systems.

\subsubsection{Mass Storage Media and Drives}

Optical disk media currently offers the highest density removable, direct-access media and was chosen as the mass storage media for the pilot. The specific optical media selected was $130 \mathrm{~mm}$ $\left(5 \frac{1}{4}\right.$ in) magneto-optic double sided media with 512 byte sectors and a capacity of 600 megabytes per cartridge. An international standard (ISO/IEC 10089) exists for this type of media, and the cost of the media, drives, and library systems were suitable for a pilot.

\subsubsection{Device Integration Method}

The device integration method includes the hardware interface as well as the software interface. The following sections discusses the Small Computer Systems Interface (SCSI), the pilot system hardware interface, and the Common Access Method (CAM), the pilot system software interface.

\subsubsection{Small Computer Systems Interface}

The Small Computer Systems Interface (SCSI) is a specification that defines a highperformance, general-purpose interface that allows a system to communicate with a wide variety of peripheral devices. Due to its flexibility, SCSI is a popular method for the integration of external mass storage devices. However, the interface has so many options that the integration of devices can be very difficult. In many cases, the physical connectors, as well as the command set used to control the devices, are vendor specific. Many "plug and play" subsystems have involved the use of proprietary boards and software, which lock a system into one manufacturer. 
In order to promote interchangeability between vendors, Task Group X3T9.2, Lower Level Interfaces, has developed the SCSI-1 and SCSI-2 standards, and is currently working on a SCSI3 standard. These standards provide specifications on the physical connectors and the command set, as well as many other characteristics that are necessary to allow for interchangeability.

SCSI compliant devices are daisy chained together and controlled by a single host bus adapter (HBA). Each device is assigned a target ID, via which the host bus adapter routes commands to the device. NIST chose to use the Western Digital 7000 FASST-2 SCSI host bus adapter.

\subsubsection{Common Access Method}

Another major problem in the integration of SCSI compliant devices is the programming interface. The Common Access Method (CAM) specification, being developed by the Task Group X3T9.2, defines a standard programming interface between an operating system and a host bus adapter. This interface provides a standard format for SCSI commands, regardless of the type of the device. For a device which is SCSI-compliant, a device driver following this specification would be written using high-level standardized commands rather than device specific SCSI commands. This enables the physical devices to be interchanged without having to rewrite code. This document is sufficiently complete that several software developers are already developing programming interfaces which comply to the CAM draft specification. NIST chose to use the Columbia Data Products SDLP CAM-compliant programming interface.

\subsubsection{Open Reel Tape Drive}

A $12.7 \mathrm{~mm}(1 / 2$ in) open reel $256 \mathrm{cpmm}$ ( $6250 \mathrm{cpi})$ 9-track tape drive was also an integral part of the pilot system as the EASEAR data was provided from SSA on this type of media. The Hewlett-Packard 88780B tape drive was selected as it provided an option for a SCSI interface.

\subsubsection{Optical Disk Library System}

In order to store all of the data intended for the pilot system, and to provide a response time acceptable for the number of users in the pilot, a library with approximately 20 gigabytes of storage capacity and two rewritable magneto-optic drives was required. It was also required that the optical drives and library cartridge handler be controlled by SCSI- 1 or SCSI-2 commands. NIST chose to use two Hewlett-Packard C1710A rack-mountable library systems. 


\section{Software Design}

The EASEAR pilot system software consists of a mix of off-the-shelf packages and software developed by NIST (see Figure 2). The off-the-shelf software consists of the ESQL/C database engine, the software drivers for the optical disk drives, as well as several UNIX utilities. The software written at NIST consists of two major programs, the EASEAR user interface and the library cartridge handler device driver. The software is written in the $\mathrm{C}$ Language, using a modular design approach, and compiled with the Interactive UNIX $\mathrm{C}$ compiler. The user interface is pre-compiled by the Informix ESQL/C precompiler, which allows the $\mathrm{C}$ language program to make SQL queries to the database engine.

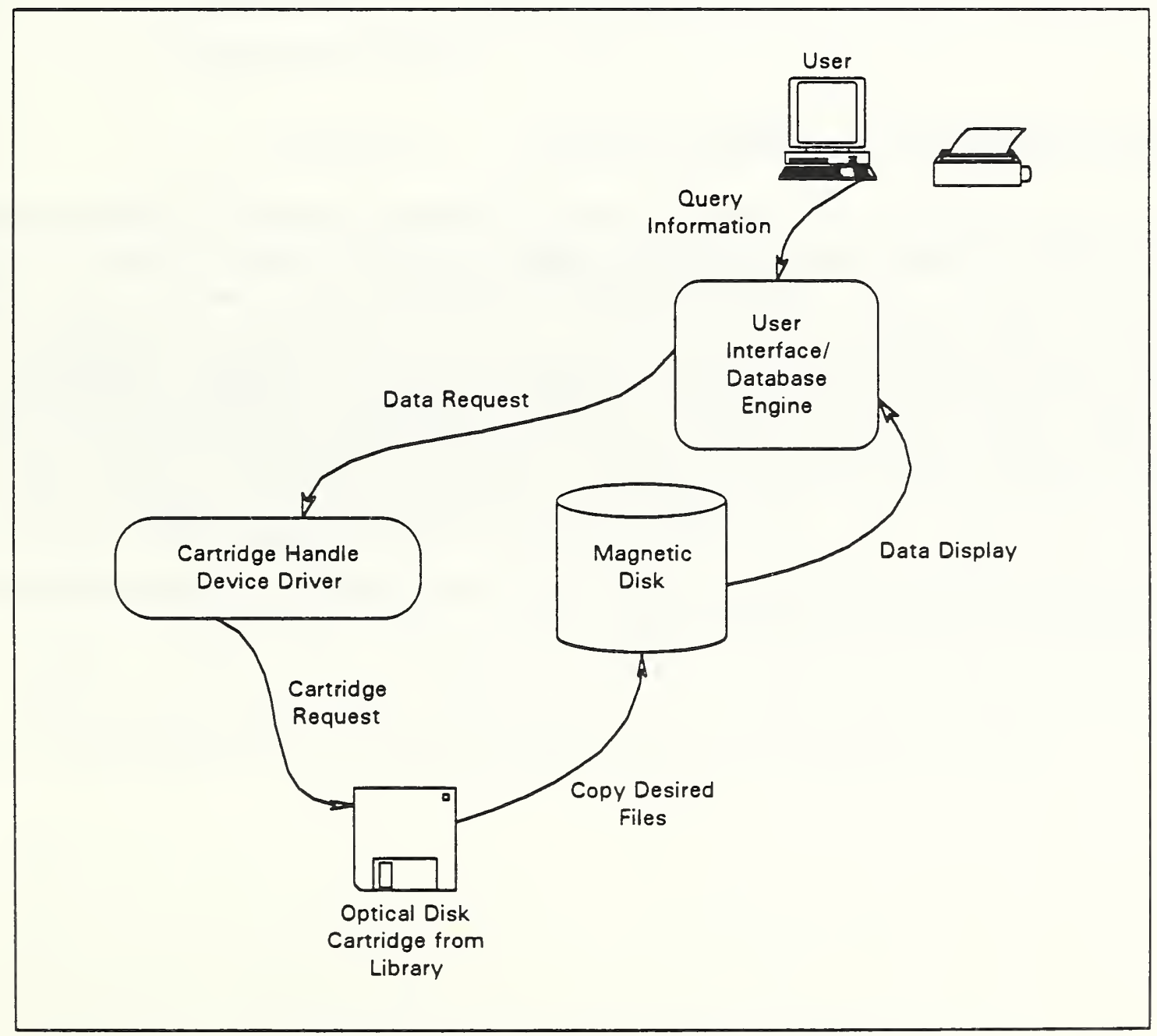

Figure 2. System Software Flow of Data 


\subsection{Overview of the EASEAR User Interface}

Before writing the pilot system user interface, several visits were made to the Metro West branch of the Social Security Administration. From information obtained on a tour of the manual retrieval system currently in use, a prototype interface was designed and implemented for an IBM PC compatible running DOS. This prototype interface was used by approximately 20 users at SSA who were asked to offer suggestions to improve the user interface design. From these comments, the prototype was refined and taken back to SSA for a final prototype test.

The EASEAR user interface provides record retrieval and viewing capabilities to the user. The user enters certain information on the record for which he or she is searching. This information is reordered into an SQL query, and records that match the query are retrieved. A limited number of fields from each record are displayed, and the user is allowed to browse through the records, select a record to be displayed in full, and select records to be printed. In addition to these functions, the user interface collects certain management information to help assess the results of the pilot system test. The listing of this code, "easear.ec", can be found in Appendix D.

\subsubsection{General Screen Characteristics}

The user interface consists of four types of screens: the query screen, the browse screen, the detail screen, and the error screen. The first screen, the query screen, is used to enter the parameters that define a query. The next screen, the browse screen, is used to view a subset of information on every record that matches the query. Both screens have a blue background and yellow text, and, in order to make available as much information as possible, are the size of the entire display. The detail screen, used to display full information on a selected record, and the error screen, used to report an error condition or status information to the user, have a red background and yellow text. These two screens are smaller than the display as only limited information is necessary, and overlay either the query or browse screen. With the exclusion of the error screen, there are prompts on the bottom of each of the screens outlining options available to the user while at the current screen. 


\subsubsection{The Query Screen}

The query screen appears upon entry into the program, and prompts the user to enter parameters for a record search (see Figure 3). It is mandatory that the user specify the tax year, the internal revenue district (IRD), and at least four letters of the wage earner's last name. Optional information includes the initials, the social security number, the spouse's social security number and the amount of self employed income.

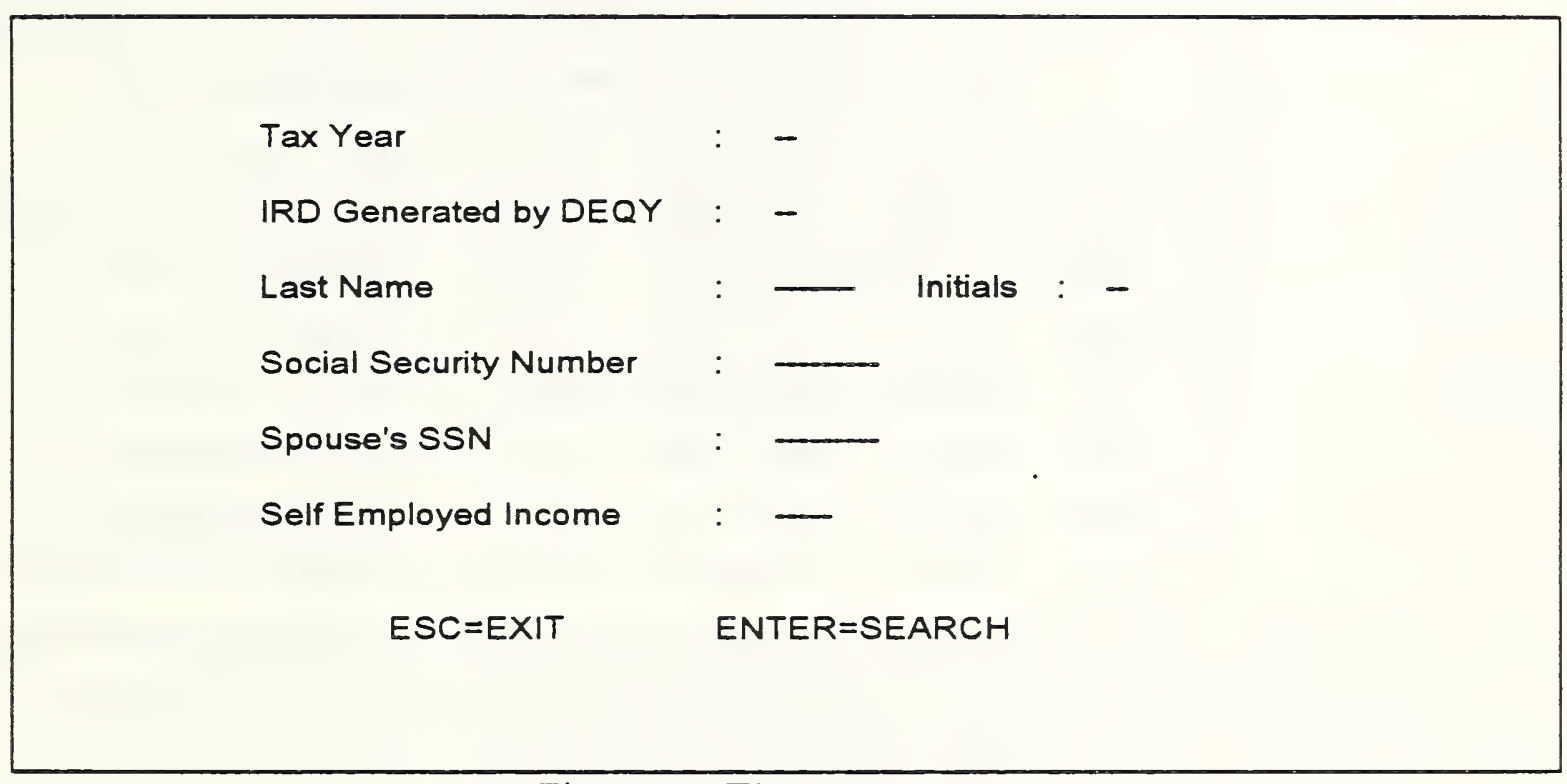

Figure 3. The query screen

If the user places a period at the end of the specified last name, only records in which the last name matches the specified pattern are retrieved. Otherwise, all records with the last name beginning with the specified character sequence are retrieved. If the user enters self employed income information, all records are retrieved in which the self employed income is in the range of $+/$ - one dollar of the figure designated as a query parameter.

When the user has entered the maximum number of characters for a response to a parameter, the cursor jumps to the next field. The user also has the use of the arrow and tab keys to move between fields. The Page Up key is used to view the tax year and the IRD from the previous query. When the user presses enter, the program retrieves the records matching the query and proceeds to the browse screen. If the user presses escape, the program terminates. 


\subsubsection{The Browse Screen}

The browse screen displays partial information on all of the records that match the user's query (see Figure 4). The information displayed is intended to be enough for the user to refine his or her search in order to request detailed information on a few records.

\begin{tabular}{|c|c|c|c|c|c|c|}
\hline \multirow{3}{*}{$\begin{array}{l}\text { Name } \\
\text { AANESTA }\end{array}$} & \multicolumn{2}{|c|}{ Tax Year: 88} & \multicolumn{4}{|c|}{ IRD Generated by DEQY: 14} \\
\hline & \multicolumn{2}{|r|}{ SSN } & SEI & \multicolumn{2}{|c|}{ Spouse's SSN and SEI } & \multirow{2}{*}{$\begin{array}{l}\text { Full Name } \\
\text { ARLET AND JAMES AANE }\end{array}$} \\
\hline & AJ & 061363003 & 37692 & 564536009 & 37347 & \\
\hline ABERNAT & JF & 658724029 & 19187 & 624052003 & 31731 & JOAN AND PETER ABERN \\
\hline ACKERMA & JA & 951812019 & 34883 & 660614017 & 19187 & JOHN AND BELINDA ACK \\
\hline ADAMSON & AJ & 658724029 & 31731 & 951812019 & 36778 & AUDREY AND CARL ADAM \\
\hline ALEXAND & $\mathrm{CC}$ & 660614017 & 24776 & 256786022 & 34883 & CHARLES AND MARY ALE \\
\hline ALEXAND & $\mathrm{CF}$ & 256786022 & 35587 & 7788940006 & 24776 & CYNTHIA AND ROB ALEX \\
\hline ALEXAND & ER & 778840006 & 33323 & 658724029 & 35587 & EUGENE AND ELEXIS AL \\
\hline ANDERSO & BJ & 624052003 & 36778 & 621690034 & 33323 & BEVERLY AND TERRY AN \\
\hline$E S C=Q L$ & ERY & CR ENTER $=\mathrm{C}$ & TAIL SC & PGUP/PGD & $=S C R O$ & L END/HOME $=S H I F T$ REC \\
\hline
\end{tabular}

Figure 4. The browse screen

The the arrow keys are used to browse through the page of records currently on the screen, and the Page Up and Page Down keys are used to retrieve a previous page or a new page of matches.

The End key is used to view any portion of the record that is too long for the standard display, and the Home key is used to restore the line.

The user is able to view all records that match the query, up to the point where system performance would be impacted. By pressing Esc, the user is returned to the query screen. If the user presses Enter while the cursor is positioned over a record, he or she proceeds to the detail screen. 


\subsubsection{The Detail Screen}

The detail screen displays all the information in the current record as it would appear on the microfilm (see Figure 5). If the user presses Enter, the record information is sent to the dot matrix printer at the user's workstation. If the user presses Print Screen, the entire screen is printed.

\begin{tabular}{|c|c|c|c|c|c|c|c|c|c|}
\hline $\begin{array}{l}\text { Name : } \\
\text { Address: }\end{array}$ & $\begin{array}{l}\text { AUD } \\
32 \mathrm{Hc} \\
\text { Gaith }\end{array}$ & $\begin{array}{l}\text { EY AND CARL } \\
\text { per Way } \\
\text { sburg, MD } 20\end{array}$ & $\begin{array}{l}\text { ADAMSO } \\
78\end{array}$ & & & & & & \\
\hline Name & & SSN & NESEB & NESEF & WAGES & SEI & YEARQU & ST & DLN/EIN IND CD \\
\hline $\begin{array}{c}\text { ADAMSON } \\
26\end{array}$ & AJ & $\begin{array}{l}061363003 \\
564536009\end{array}$ & $\begin{array}{l}00000 \\
00000\end{array}$ & $\begin{array}{l}00000 \\
00000\end{array}$ & $\begin{array}{l}00000 \\
00000\end{array}$ & $\begin{array}{l}37692 \\
37347\end{array}$ & 8812 & 21 & $\begin{array}{l}16222111940356 \\
0607676257716\end{array}$ \\
\hline
\end{tabular}

Figure 5. The detail screen

\subsubsection{The Error Screens}

The error screens are used to inform the user that an error condition has occurred (see figure 6). When an error screen appears, the user presses Esc to exit the screen. Some error messages are caused by an improper entry, and return the user to the current screen. Others are system error messages, and cause program termination. Refer to Appendix C for a list of error messages.

Error! You Must Enter the Tax Year, IRD and 4 Letters of the Last Name

Figure 6. The error screen

\subsubsection{Management Information}

The EASEAR user interface maintains certain management information in order to help assess the results of the pilot system test. It collects the information, and stores it in a file. A separate program uses the information stored by the user interface and prints the following:

- Total logins to the pilot system

- Total time elapsed during all logins

- Average time elapsed during a login

- Total number of queries

- Total number of 1988 queries

- Total number of 1989 queries

- Total time elapsed during all queries

- Total time elapsed during 1988 queries

- Total time elapsed during 1989 queries

- Average time elapsed during a query
- Average time elapsed during a 1988 query

- Average time elapsed during a 1989 query

- Total requests for detailed records

- Total requests for 1988 detailed records

- Total requests for 1989 detailed records

- Total requests for record printouts

- Total requests for 1988 record printouts

- Total requests for 1989 record printouts 


\subsection{User Interface Software Organization}

The following subsections discuss the data structures implemented in the user interface, as well as the high level software organization.

\subsubsection{Software Data Structures}

The major data components involved in the user interface consist of seven data structures: the query parameters, the browse data, the full record data, the Informix host variables, the request message, the acknowledge message, and the management information.

\subsubsection{The Query Parameters}

The Query Parameters data structure is implemented as an array of size two of the user-defined $\mathrm{C}$ structure INFO, and is used to store search parameters entered by the user. One element of the array is used to store the current query parameters, while the other array element is used to store the previous query parameters. The structure contains the following data:

- Tax Year

- IRD

- Last name

- Initials
- Social Security Number

- Spouse's Social Security Number

- Self Employed Income

\subsubsection{The Browse Data}

The Browse data structure is implemented as an array of size MAXSCR (number of matches displayed on the screen in the user interface) of the user-defined C structure QUERY, and is used to store the fields of the record that the user views in the browse screen. The structure contains the following data:

- Tax Year

- IRD

- Last Name

- Initials

- Social Security Number
- Self Employed Income

- Spouse's Social Security Number

- Spouse's Self Employed Income

- Full Name as Reported on the 1040 form 


\subsubsection{The Full Record Data}

The Full Record data structure is implemented as an array of size MAXSCR (number of matches displayed on the screen in the user interface) of the user-defined C structure REC, and is used to store the remainder of the record fields which the user views in a detail screen. The structure contains the following data:

$\begin{array}{ll}\text { - NESEB } & \text { - Spouse's NESEB } \\ \text { - NESEF } & \text { - Spouse's NESEF } \\ \text { - Wages } & \text { - Spouse's W Wages } \\ \text { - Month } & \text { - Date } \\ \text { - Quarter } & \text { - EIN } \\ \text { - Option Code } & \text { - Industry Code } \\ \text { - DLN } & \text { - Street Address } \\ \text { - IWP } & \text { - City and State } \\ \text { - Error Code } & \text { - Zip Code }\end{array}$

\subsubsection{The Informix Host Variables}

The Informix Host variables data structure is implemented as the user-defined $\mathrm{C}$ structure RECEIVE, and is precompiled by the Informix ESQL/C database engine. It is used as a set of variables for the passing and receiving of data between the user interface and the embedded database engine. This is a separately defined data structure because the database engine has problems dealing with an array of structures serving as a host variable. It contains all of the information in the QUERY and REC user-defined structures.

\subsubsection{The Request Message}

The Request Message data structure is implemented as the user-defined C structure MSGBUF, and is used to pass requests for mounting and unmounting cartridges to the cartridge handler device driver. The structure contains the following data:

- Message Type (mount or unmount)

- User Number

- Library Number

- Cartridge Number

- Cartridge Side 


\subsubsection{The Acknowledge Message}

The Acknowledge Message data structure is implemented as the user-defined C structure MSGACK, and is used to receive acknowledgement of a mounted cartridge from the cartridge handler device driver. The structure contains the following data:

- Message Type (Acknowledge)

- Directory to which the cartridge has been mounted

\subsubsection{The Management Information}

The Management Information data structure is implemented as the user-defined $\mathrm{C}$ structure MANAGEINFO, and is used to collect information that will be used to assess the effectiveness of the pilot system. The structure contains the following data:

- Number of logins to the pilot system

- Length of time the user has been logged into the pilot system

- Number of 1988 and 1989 queries

- Length of time the user is involved in a 1988 and 1989 query

- Number of 1988 and 1989 detailed screens viewed

- Number of 1988 and 1989 printouts requested

A separate program, "print_mi.c", uses this information to calculate and print the parameters listed in section 4.1.6. The code for this program is listed in Appendix D. 


\subsubsection{High Level Software Organization}

The user interface software organization consists of five distinct phases: the initialization and control of the user environment, the entry of the user query, the selection of the matches to the query, the display and viewing of the browse fields, and the display of detailed record information (see Figure 7). These phases are discussed in the following sections.

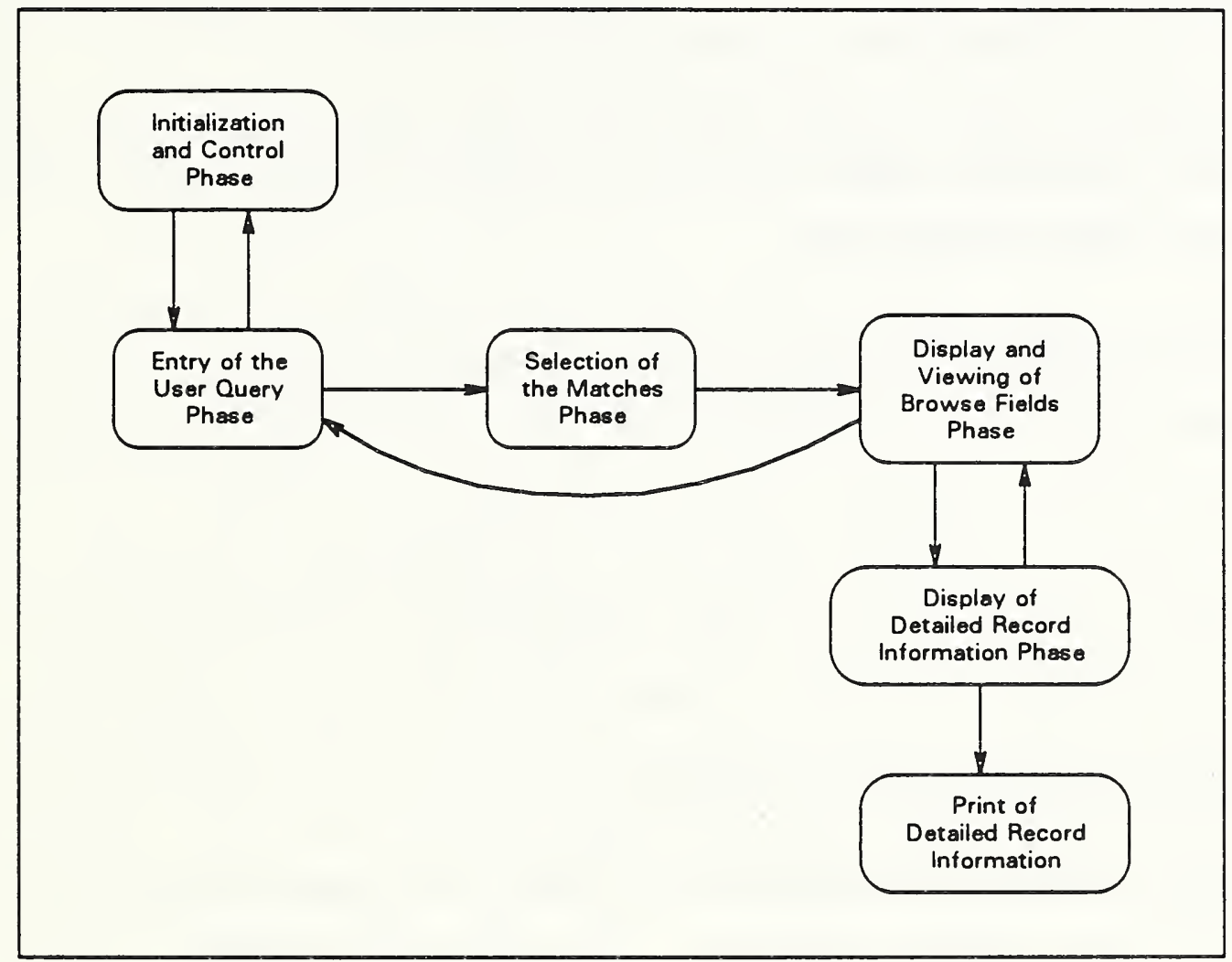

Figure 7. User interface software control flow

\subsubsection{Initialization and Control of the User Interface Environment}

The user interface uses a set of library routines in the UNIX compiler referred to as the "curses" library. These routines provide windowing, screen handling, and cursor control functions, and are used to draw and manage the user interface screens for character oriented displays. In the initialization phase, the screen windows are set up, the management information is initialized, the user number is determined based on the port the user is logged into, the length of login timer is started, and the login count is incremented.

The entry of the query phase is then entered by a call to the get_query 0 function. Control is returned to initialization phase when the get query 0 function returns an escape character, signalling the end of a session. At this point, the login timer is ended, the management information is collated and written to a file, the windowing environment is exited and the program terminates. 


\subsubsection{Entry of the User Query}

In the Query Entry Phase, the get_query 0 function performs the following steps:

- initializes the length of query timer

- increments the query count

- draws the query screen

- traps and interprets each character entered by the user

If the user presses Esc, the program returns to the initialization phase and the query timer is discarded. If the user presses Enter, the select_matches 0 function is called, and the program proceeds to the match selection phase.

All characters, excluding escape and enter, pass to the function interp 0 , which either processes the input or routes it to the correct lower level function. If the character is an arrow key, the character passes to the proc_arrow ( function, which moves the cursor in the direction of the arrow. If the character is a tab key, the cursor moves to the next field. If the character is a Page Up key, the tax year and the IRD from the previous query are displayed. If the character is a backspace, it passes to the function del_char0, which deletes the last character entered, from both the screen and the INFO data structure. Otherwise, the character passes to the function add_char 0 , which adds the character to both the screen and the INFO data structure.

\subsubsection{Selection of the Matches to the Query}

In the Selection of Query Matches Phase, the INFO data structure containing the user query passes to the select matches 0 function. The last name parameter is checked by the check_search_type 0 function to determine if the search type is prefix or exact match. The assign_user_files 0 function is called to allocate files for use by this user, and the req_user_data 0 function is called to request files from the optical disk library.

The req_user_data 0 function queries the indexing tables for files that contain the IRD, last name and initials, if specified, that match the data in the INFO structure. If the number of files that meet this criteria is greater than twenty, an error occurs and the user is asked to refine his or her query and control returns to the entry of the query phase. Otherwise, the cartridge containing the files is requested from the cartridge handler scheduling algorithm. Upon receipt of an acknowledge message, the files are copied from the cartridge to the magnetic hard disk and decompressed, and the scheduling algorithm is notified that the use of this cartridge is complete. The program then returns to the select_matches 0 function.

At this point, the data in the INFO structure are concatenated into a string which forms the SQL query. The user's database is then opened, and all matches to the query are retrieved. If no matches are found, an error message is displayed, and program returns to get_query function 0 and the entry of the query phase. If matches are found, the display_matches 0 function is called, and control of the program continues to the display and viewing of the browse fields. 


\subsubsection{Display and Viewing of Browse Fields}

In the Display and Viewing of Browse Fields Phase, the display_matches 0 function draws the query screen and each character entered by the user is trapped and interpreted. If the user presses Esc, the length of query timer is ended, and the program returns to the get_query 0 function and the query entry phase. If the user presses enter, the disp_rec 0 function is called, and the program proceeds to the detail view phase.

All characters, excluding escape and enter, are passed to the function browse 0 , which either processes the input or route it to the correct lower level function. If the character is an arrow key, the cursor is moved in the direction of the arrow. If the character is a page up, the view previous matches 0 function is called and the previous page of matches is retrieved, if applicable. If the character is a page down, the view_next_matches 0 function is called and the next set of matches is retrieved, if applicable. If the character is an end key, the move line 0 function is called and the rightmost portion of the current record is displayed. If the character is a home key, the restore line 0 function is called and the leftmost portion of the current record is displayed.

\subsubsection{Display of Detailed Record Information}

In the display of detailed record information phase, the disp_rec 0 function draws the detail screen, increments the detail view count, and both traps and interprets each character entered by the user. If the user presses Esc, the program returns to the display matches 0 function and the Query Entry Phase. If the user presses Enter, the program calls the print_rec 0 function, increments the print count, and prints the record. All other characters are disregarded.

\subsection{Overview of the Cartridge Handler Device Driver}

The Helwett-Packard optical disk library system contains two $130 \mathrm{~mm}$ ( $51 / 4 \mathrm{in}$ ) magneto-optic drives, a cartridge handler device and 32 cartridge slots. The Columbia Data Products software drivers, in combination with several UNIX utilities, provide adequate support for the two drives.

No off-the-shelf software support existed for the cartridge handler device in this environment, therefore it was necessary to develop the software at NIST. The device driver consists of two major algorithms: a scheduler algorithm and a control algorithm. The scheduler algorithm creates and maintains a messaging interface with all copies of the EASEAR user interface that are running, and handles their requests for optical disk cartridges. The control algorithm deals with the movement of the cartridge handler device. The listing of this code, "devhandler.c", can be found in Appendix D. 
The cartridge handler device driver must be run with system administrator privileges in the background. This is necessary in order to have the correct permissions to mount and unmount file systems.

\subsubsection{The Scheduler Algorithm}

The scheduler algorithm communicates with the EASEAR user interface through a form of UNIX interprocess communication referred to as messaging. After setting up the messaging interface, the scheduler waits for a request from the user interface. The messages are one of two types - a request to mount a cartridge or to unmount a cartridge.

Before checking the incoming messages, the scheduler first checks to see if any drives are available. If no drives are currently free, the scheduler waits until an unmount request is placed in the queue and services it before retrieving any mount requests.

When a mount request is received, the scheduler checks to see if the cartridge is available. If so, a drive is allocated, the cartridge is moved into the drive and mounted, and an acknowledge message is sent to the user interface. If the requested cartridge is currently in use, the request is placed in the wait queue.

When an unmount request is received, the scheduler first checks the wait queue to see if any requests are pending for the cartridge in question. If there are no requests, the cartridge is unmounted and returned to its library slot. If a request is pending, the scheduler ascertains which side of the cartridge is requested, flips the cartridge if necessary, and sends an acknowledge message to user interface.

\subsubsection{The Control Algorithm}

The cartridge handler device is controlled through the Columbia Data Products Standard Level Device Protocol (SDLP) interface. This interface, for supported types of devices, removes the necessity to send SCSI commands to the device. Instead, higher level commands are sent to an SDLP driver which generates the SCSI commands. As the cartridge handler is not currently a supported device for the SDLP interface, it was necessary to use the pass-through mode to bypass the SDLP interface and send SCSI- 2 commands directly to the cartridge handler.

When the scheduling algorithm requests that a cartridge be moved, the control algorithm creates a command descriptor block (CDB) that contains the SCSI-2 command for move medium, and sends an SDLP command to the Columbia Data Products SDLP driver requesting that this CDB be passed through to the cartridge handler device. 


\subsection{Cartridge Handler Device Driver Software Organization}

The following subsections discuss the data structures implemented in the cartridge handler device driver, and the high level software organization.

\subsubsection{Software Data Structures}

The major data components involved in the cartridge handler device driver consist of six data structures: the message queues, the request message, the acknowledge message, the drive status information, the command descriptor block, and the SDLP command information.

\subsubsection{The Message Queues}

The Message Queues are implemented as a $C$ system-defined structure, and are managed by the UNIX operating system. The messages in the queue are implemented as a system-defined linked list that is also managed by the UNIX operating system. The exact format of these data structures is found in the UNIX C library header files "ipc.h" and "msg.h". For the purposes of this documentation, the queues and messages are viewed as a structure pointer that is returned by the system.

The cartridge handler device driver utilizes three message queues -- the incoming messages queue, the wait queue, and the acknowledge queue. The incoming messages queue is used to receive cartridge requests from the user interface. The wait queue is used to hold cartridge requests that cannot be filled immediately due to the cartridge being in use. The acknowledge queue is used to send acknowledgement of an available cartridge to the user interface.

These queues are created by the scheduler but are not released by the program as the scheduler operates continuously. If, for any reason, the scheduler is terminated, it is necessary to release the messaging queues before restarting the program. There is a small program, "killq.c", which releases the queues. The listing of this code is located in Appendix D.

\subsubsection{The Request Message}

The Request Message data structure is implemented as the user-defined C structure MSGBUF, and is used to receive requests from the user interface for the mounting and unmounting of cartridges. The structure contains the following data:

- Message Type (mount or unmount)

- User Number

- Library Number

- Cartridge Number

- Cartridge Side 


\subsubsection{The Acknowledge Message}

The Acknowledge Message data structure is implemented as the user-defined C structure MSGACK, and is used to send acknowledgement of a mounted cartridge to the user interface. The structure contains the following data:

- Message Type (Acknowledge)

- Directory to which the cartridge has been mounted

\subsubsection{The Drive Status Information}

The Drive Status information is implemented as an array size MAXDRIVES (the number of drives in the pilot system) of the user-defined C structure DRINFO, and is used to maintain the current state of each of the drives, as well as system and address information on the drive. The following information is kept for each of the drives:

- The SDLP unit number of the jukebox in which it resides

- The SDLP unit number of the drive

- The file name of the software driver for the drive

- The directory to which this drive mounts a cartridge

- A flag indicating whether or not the drive is in use

- The cartridge number of the cartridge currently in the drive

- The side of the cartridge currently in the drive

\subsubsection{The Command Descriptor Block}

The Command Descriptor Block is implemented as an array size 12 of characters, and is used to store the move medium SCSI command and related information needed by the cartridge handler device. It contains the following information:

- SCSI command (0xA5 - move media command)

- Logical Unit Number of target device

- Cartridge Handler Device Address

- Source Element Address

- Destination Element Address

- Invert Flag 


\subsubsection{The SDLP Command Information}

The SDLP Command Information is implemented as a user-defined C structure, and is used to store information needed by the Columbia Data Products SDLP driver in order to execute a passthrough move medium SCSI command. The structure contains the following information:

- Command for the SDLP device driver to execute (0xff - passthrough)

- SDLP unit number of the device for which this command is intended

- Address of the return buffer (for commands that return information)

- Length in bytes of the return buffer

- Address of the Command Descriptor Block containing SCSI command

- Length in bytes of the Command Descriptor Block

- I/O direction of the command (read or write)

- Type (always 0)

- Error Status

\subsubsection{High Level Software Organization}

The cartridge handler device driver organization consists of three distinct phases: the initialization of the data structures, the receipt of a request, and the processing of a request (see figure 8). These phases are discussed in the following sections.

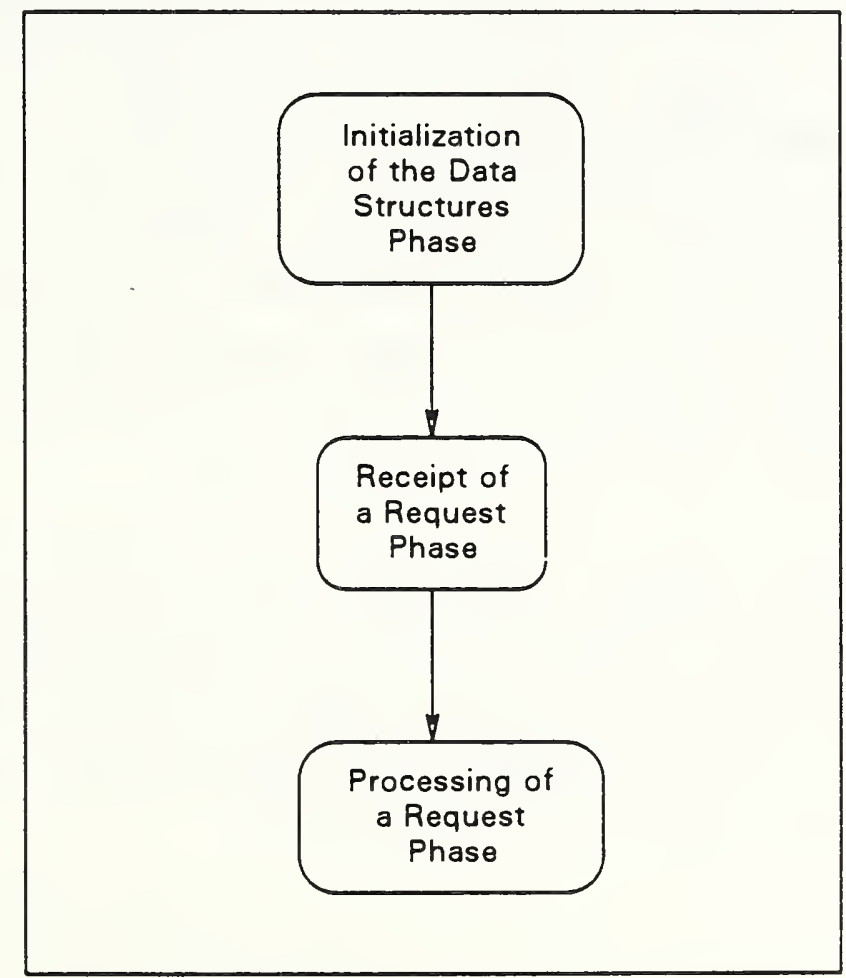

Figure 8. Cartridge handler device driver software control flow 


\subsubsection{Initialization of the Data Structures}

In the initialization phase, the messaging environment is created and the control data structures are initialized. The incoming messages, wait, and acknowledge queues are created, the SDLP device driver is opened, and the DRINFO data structure is initialized to contain starting information for each of the drives. Control passes to the receipt of a request phase, which runs continuously.

\subsubsection{Receipt of a Request}

In the receipt of a request phase, the DRINFO data structure is checked to determine if any drives are available. If a drive is free, the program calls the msgrev $($ function and waits for the next message to be placed in the queue. If no drives are free, the msgrcv $O$ function waits until the next unmount message is placed in the queue. Once a message is received and stored in the MSGBUF data structure, control passes to the processing of a request phase.

\subsubsection{Processing of a Request}

In the processing of a request phase, the MSGBUF data structure is checked to determine which kind of request has been received.

If the request is a mount request, the DRINFO data structure is checked to determine if the requested cartridge is free. If it is currently in use, the MSGBUF data structure is sent to the msgsnd $O$ function and the message is placed in the wait queue. If the cartridge is free, the free_drive 0 and mark_busy $O$ functions are called to allocate a drive, and the mov_disk 0 function is called in order to move the cartridge to the allocated drive. At this point, a hardware response delay is caused to allow the cartridge to be inserted into the drive, and then the mount disk 0 function is called to mount the file system. If the process is not delayed, the mount_disk 0 function will fail. Once the file system is mounted, the MSGACK data structure is filled and sent to the msgsnd $($ function and the message is placed in the acknowledge queue. At this point, control is returned to the receipt of a message phase.

If the request is an unmount request, the determine drive 0 function is called to ascertain which drive contains the cartridge to unmount. The check__wait_queue 0 function is then called to see if any messages in the wait queue need this cartridge. If not, the mark idle 0 function is called to change the status of the drive to idle, the unmt disk 0 function is called to unmount the file system, and the ret_disk 0 function is called to return the cartridge to the library slot. At this point, control is returned to the receipt of a message phase. 
If a message in the wait queue is waiting for this cartridge, the MSGBUF data structure is filled with the pending message, and is checked to determine if the current side of the cartridge is needed. If so, the MSGACK data structure is filled and sent to the msgsnd 0 function and the message is placed in the acknowledge queue. If the opposite side is needed, the unmt disk 0 function is called to unmount the file system, the mark busy 0 function is called to update the cartridge information stored in the DRINFO data structure, the flip_disk 0 function is called to flip the cartridge in the drive, and, after a short hardware response delay, the mount disk 0 function is called to mount the file system. The MSGACK data structure is filled and sent to the msgsnd 0 function and the message is placed in the acknowledge queue. At this point, control is returned to the receipt of a message phase. 


\section{Conversion and Handling of SSA Data}

The EASEAR data was received from SSA on $12.7 \mathrm{~mm}(1 / 2$ in) open reel $256 \mathrm{cpmm}$ (6250 cpi) 9-track tape. Each year of the data contained approximately 11 million records, and was stored as a multi-volume file split over 17 tapes. The data was in the EBCDIC character set, with each tape containing a header section, a data section, and a trailer section. Each section is separated from the others with filemarks. The tapes contained between 60 to 107 blocks, with 31824 bytes each and separated with a $1<$ character. The data itself was split into records of 234 bytes each, and was organized in increasing numeric order by IRD, and in alphabetic order within IRD.

The EASEAR data was manipulated at NIST until it was finally stored as database files of 300 records using the ASCII character set. Each of the files contains only one IRD, and is assigned a name beginning with the IRD and a sequential file number starting at one for each IRD. The files are stored on $130 \mathrm{~mm}\left(5 \frac{1 / 4}{4}\right.$ in) magneto-optic cartridges in a compressed format. In its final compressed state, one year of data consumes approximately one gigabyte of optical disk storage.

The steps in the conversion of the data include the following (see Figure 9):

- transfer the data from tape to optical disk cartridge

- convert the data to the ASCII character set

- analyze the data to determine which IRD's resided in each of the files

- separate the data into files of 300 records, with only one IRD per file

- convert the data to Informix Relational Database Table format

- compress the database tables 


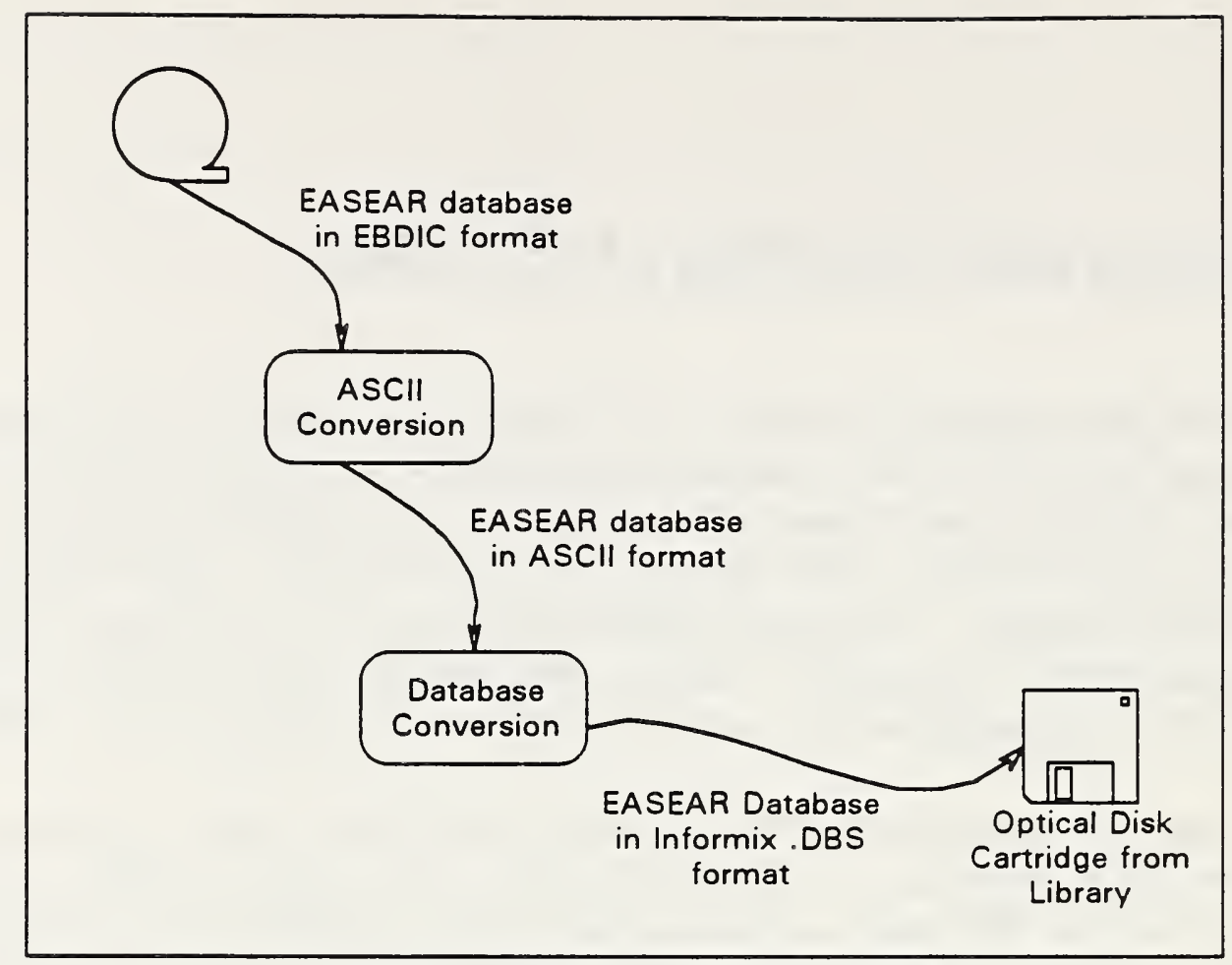

Figure 9. Data conversion process

\subsection{Basic Procedures}

There are some basic system procedures required for the system manager to convert the data These include inserting a cartridge into a library drive, ejecting a cartridge from a library drive, logging into an account on the UNIX system, gaining system administrator privileges, formatting an optical disk cartridge, placing a file system on an optical disk cartridge, mounting a file system, unmounting a file system and logging out of an account.

\subsubsection{Inserting a Cartridge into a Library Drive}

Insert the cartridge into the library mailbox slot. The side of the cartridge to be used by the drive should be facing to the right. Press the load button and then use the next and previous buttons until the desired drive number is shown in the display. Press the enter button. The cartridge is loaded into the selected drive.

\subsubsection{Ejecting a Cartridge from a Library Drive}

Press the eject button to remove the cartridge from the drive, and use the next and previous buttons to select the correct drive. Press the enter button. The cartridge is unloaded from the selected drive and placed in the library mailbox slot. 


\subsubsection{Logging into an Account on the UNIX System}

At the login: prompt, type the account name. The system prompts for the password. Once the valid password is entered, the system prompt, a \%, is displayed.

\subsubsection{Gaining System Administrator Privileges}

At the system prompt, type: su root. The system prompts for the password to this account. Once the valid password is entered, the root system prompt, a \#, is displayed.

Note: The super-user account is a privileged account on the UNIX system. While logged in as the super-user, there are no restrictions on what can be done to the system. Extreme caution should be exercised while in this account.

\subsubsection{Formatting an Optical Disk Cartridge}

Place the cartridge into the library drive, and log into the system. At the system prompt, type: /usr/sst/dutil. At the menu, select the device corresponding to the drive in which the cartridge resides. When prompted to select an action, choose option 7 , Format. When asked to enter an interleave value, enter $\mathbf{0}$, Default interleave value. When asked whether to continue with format or to quit, choose Yes, continue with format.

The screen says: Formatting Device \#... and when the format is complete, the screen says: Format of Device \# Successful, press any key.

When asked to select an action, choose option 9 to exit, then choose $Q$ to quit. At the "\%" prompt, log out of the system and remove the cartridge from the library drive.

\subsubsection{Placing a File System on an Optical Disk Cartridge}

Place the cartridge into the library drive, and log into the system. At the system prompt, if the cartridge is in drive number one, type: $\mathrm{mkfs} / \mathrm{dev} / \mathrm{sstb0} 576998$. If the cartridge is in drive number two, type: mkfs /dev/sstb1 $\mathbf{5 7 6 9 9 8 .}$

The screen prompts with $m k f s / d e v / s s t b x$ (Del if wrong) and pauses for 10 seconds. Press the delete key if the wrong device name has been entered, and re-enter the command as above. 
If the process is not stopped, the screen displays file size information such as logical block size, number of logical blocks, etc., and places a file system on the cartridge. This takes approximately 15 minutes. When completed, the screen displays the total available blocks and returns to the "\%" prompt. Log out of the system and remove the cartridge from the library drive.

\subsubsection{Mounting a File System}

To mount a file system, the system manager must have system privileges. Insert the cartridge into the library drive. At the \# prompt, type: mount /dev/sstbx /directory, where $\mathbf{x}$ is 0 if the cartridge is in drive one or 1 if the cartridge is in drive two, and directory is the name of the directory to which to mount the cartridge in the drive.

The system then mounts the file system on the cartridge to the specified directory. This takes approximately 5 minutes. When complete, the screen displays warning: $<>$ mounted as $</$ drive $0>$.

\subsubsection{Unmounting a File System}

To unmount a file system, the system manager must have system privileges. At the \# prompt, type: umount / $\mathbf{d e v} / \mathbf{s s t b x}$ where $\mathbf{x}$ is 0 if the cartridge is in drive one, or 1 if the cartridge is in drive two. The system then unmounts the file system. This takes approximately 5 minutes.

\subsubsection{Logging out of an Account}

At the system prompt, type: exit to leave the account. 


\subsection{Conversion from Tape to Optical Disk}

The first step in the conversion of the EASEAR data was to copy the data from tape to optical disk. The data were also converted to the ASCII character set, and split into smaller files.

Moving the data to optical disk rather than leaving it on magnetic tape made the data conversion process much easier for our system as this provided direct-access retrieval of the data. The data were copied from the tape and converted to ASCII using the UNIX utility "dd". The actual code for this procedure is the shell script "downld", a listing of which is in Appendix D.

The script deals with the data in 50 block sets of data. The information is read from the tape, converted to ASCII, and stored in a file on the optical disk whose file name corresponds to the current value of the loop counter within the script. The resulting file size on the optical disk is approximately 1.5 megabytes.

\subsubsection{System Procedures}

Take the tape drive off-line, and press Unload to open the tape hatch. Remove the tape from its case and insert the tape into the drive. Close the hatch, and wait for the tape to load. The display on the drive reads Ready when complete.

Place the cartridge into the library drive, and log into the system. At the \% prompt, type: /usr/sst/dutil. At the menu, when prompted to select a device, choose the number of the device corresponding to the tape drive. When prompted to specify an action, choose option 6, File mark. When asked to go to a file mark or to write a filemark, choose option $\mathbf{g}$, Go to a file mark.

The screen displays Searching for filemark, and once it is found, displays Filemark found. When asked to select an action, choose option 9 to exit and then choose option Q to quit.

Mount the destination cartridge to /drive0, and type: downld (\#sets), where a set is defined as 50 blocks of data on the tape. Round the value up to the nearest integer. This converts the tape data from EBCDIC to ASCII, and stores the data on the optical cartridge with a filename corresponding to the set number. The results of the transfer of each set are printed on the screen.

Unmount the cartridge from the library drive, remove the cartridge from the library drive, and log out of the system.

Take the tape drive off-line, and press the rewind/unload button on the drive twice to rewind and unload the tape. The tape hatch door opens when rewind/unload is complete. 


\subsection{Data Analysis}

The next step in the data conversion was to analyze each of the files in order to determine which IRD's resided in each of the files, and to create a file listing the IRD's found in each file.

The program reads in each record, one at a time, and checks the value of the IRD in the record If the IRD is the same as that of the previous record, the program continues to the next record. If the value is different from the previous record, the IRD and the current filename is written to a file which is named irdfile.x, where $x$ stands for the tape number (see Figure 10). This analysis is done to expedite the next process, the separating of the data into smaller files by IRD. The code for this procedure is the C program "check.c", listed in Appendix D.

\begin{tabular}{|ll|}
\hline 01 & /drive2/0 \\
01 & /drive2/1 \\
01 & /drive2/2 \\
01 & /drive2/3 \\
01 & /drive2/4 \\
01 & /drive2/5 \\
01 & /drive2/6 \\
01 & /drive2/7 \\
01 & /drive2/8 \\
01 & /drive2/9 \\
01 & /drive2/10 \\
02 & /drive2/10 \\
02 & /drive2/11 \\
\hline
\end{tabular}

Figure 10. IRD file

\subsubsection{System Procedures}

Insert the cartridge into the library drive, log into the system, and mount the cartridge to /drive 0 .

At the \% prompt type: ls $-1 /$ drive0. This provides a directory of the files on the cartridge. Look for the highest numbered file, and add one to this number. This is the number of files to analyze.

At the \% prompt, type: check. The program prompts for the number of files to analyze, and the tape number from which this data originated.

The program analyzes each of the files in turn and a list of the IRD's contained in each file is written to the file "irdfile.x", where $x$ corresponds to the tape number.

Unmount the file system, remove the cartridge from the library drive, and log out of the system. 


\subsection{Separation of the Data}

The data was then separated into files of 300 records in length. This number of records was chosen to provide maximum effectiveness for compression and decompression, both in speed and size, as well as trying to transfer from optical disk cartridge the fewest number of records in order to meet a user's queries.

An index file, startend. $x$ where $x$ is the IRD, is generated for each of these files (see figure 11). The index file contains the starting last name and initials, the ending last name and initials, and the filename of each output file. The code for this procedure is the C program "sep.c" listed in Appendix D.

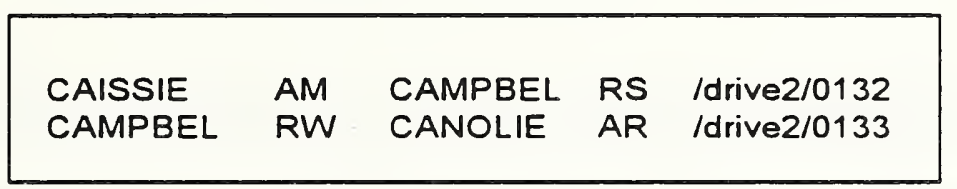

Figure 11. Index file

The program accepts as input a starting filename and the total number of files to search. For each of the files, the records are read from the input file and written to an output file. A record count is maintained, and when the number of records written to one output file reaches 300 , a new output file is started. The output files are named with the IRD and a sequential file number that starts at zero for each IRD. An entry is made in the IRD's index file for the first record and the last record of each output file. Records that contain an IRD lower than the IRD for which the program is searching are skipped. When a record is encountered with an IRD higher than the search IRD, the program terminates.

\subsubsection{System Procedures}

Place the source cartridge in the library drive two, and the destination cartridge in the library drive one. Login to the system and mount the source cartridge to /drive2 and the destination cartridge to /drive1.

At the \% prompt, type: sep. When prompted, enter the IRD for which to search, the total number of files to search, and the file number with which to start. This information may be obtained from the data analysis report generated in the previous step. 
The data that corresponds to the selected IRD is extracted from the larger files and stored on the destination cartridge in small files. At the same time, indexing information for this IRD is stored in a file called "startend.x", where $x$ corresponds to the IRD. If the data for this IRD is contained on more than one cartridge, the system manager is prompted to change cartridges. Go to another terminal, unmount and remove the current cartridge, insert and mount the next cartridge. The system manager is again be prompted for the number of files to search, and the file number with which to start.

Unmount the file systems, remove the cartridges from the library drives, and log out of the system.

\subsection{Conversion of Data to Informix Database Table Format}

The files separated by IRD are then converted to Informix database table format, and indexed by last name. The program accepts as input a list of file names, and for each file creates a database and an index file. The records are then read in one by one and stored in the table. The database table and index file are copied to the destination cartridge, and the table on the hard drive is dropped. The code for this procedure is the C program "conv.ec", listed in Appendix D.

The last name and initials index files generated in the separating step are converted to database table format and stored on the magnetic media. In addition, fields are added to these tables to indicate on which cartridge and side the data are stored. The code for this procedure is the $C$ program "index.ec", listed in Appendix D.

\subsubsection{System Procedures}

Place the source cartridge in the library drive two, and the destination cartridge in the library drive one. Login to the system and mount the source cartridge to /drive2 and the destination cartridge to /drivel.

At the \% prompt, type: ls $>/$ usr/pilot/names. This generates the list of input file names that the program expects.

Type: conv Each file is converted and the database and index files are copied to the destination cartridge. 
Unmount the file systems, remove the cartridges from the library drives, and log out of the system.

\subsection{Compression of the Data}

The last step in the data conversion process is the compression of the database and index files. The compression algorithm used is the Huffman encoding scheme, which is included in the UNIX operating system as a utility. The code for this step is the c program "compress.c" listed in Appendix D.

\subsubsection{System Procedures}

Place the source cartridge in the library drive two, and the destination cartridge in the library drive one. Login to the system and mount the source cartridge to /drive 2 and the destination cartridge to /drive 1 .

At the \% prompt, type: cd /drive2, then type: Is > /usr/pilot/names and then type: cd /usr/pilot. This generates the list of input file names that the program expects.

Type: comp < names. Each file is copied to the destination cartridge and compressed.

Unmount the file systems, remove the cartridges from the library drives, and log out of the system. 


\section{References}

American National Standard for Information Systems - Small Computer Systems Interface (SCSI), ANSI X3.131-1990.

Federal Information Processing Standard Publication - Database Language SQL, FIPS Publication 127-1.

International Standard ISO/IEC 10089, Part A, Information Technology - 130 mm Rewritable Optical Disk Cartridges for Information Interchange.

SCSI Common Access Method - Transport and SCSI Interface Draft Document, X3T9.2 90186 Revision 2.3.

Federal Information Processing Standard Publication - POSIX: Portable Operating System Interface for Computer Environments, FIPS Publication 151-1. 


\section{Appendix A}

\section{User Instructions}

\section{A.1 Entering the System}

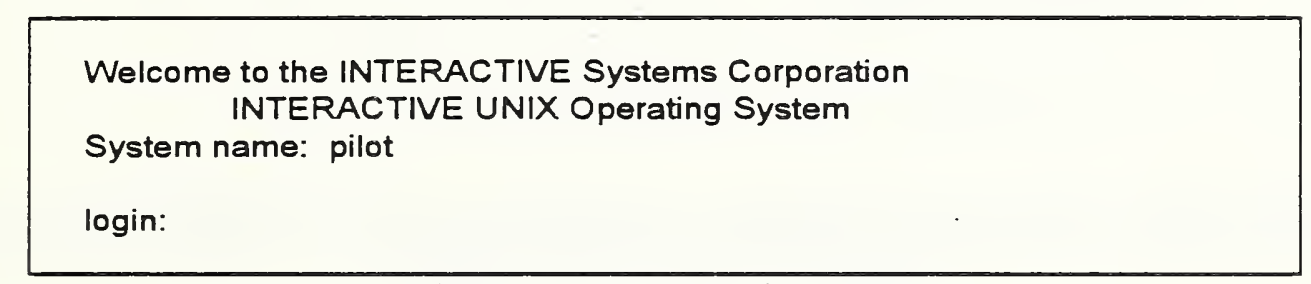

Figure Al. The login screen

In Order to ...

- Login to the system, at the login: prompt, type the account name. At the Password: prompt, type the password for the account. 


\section{A.2 The Query Screen}

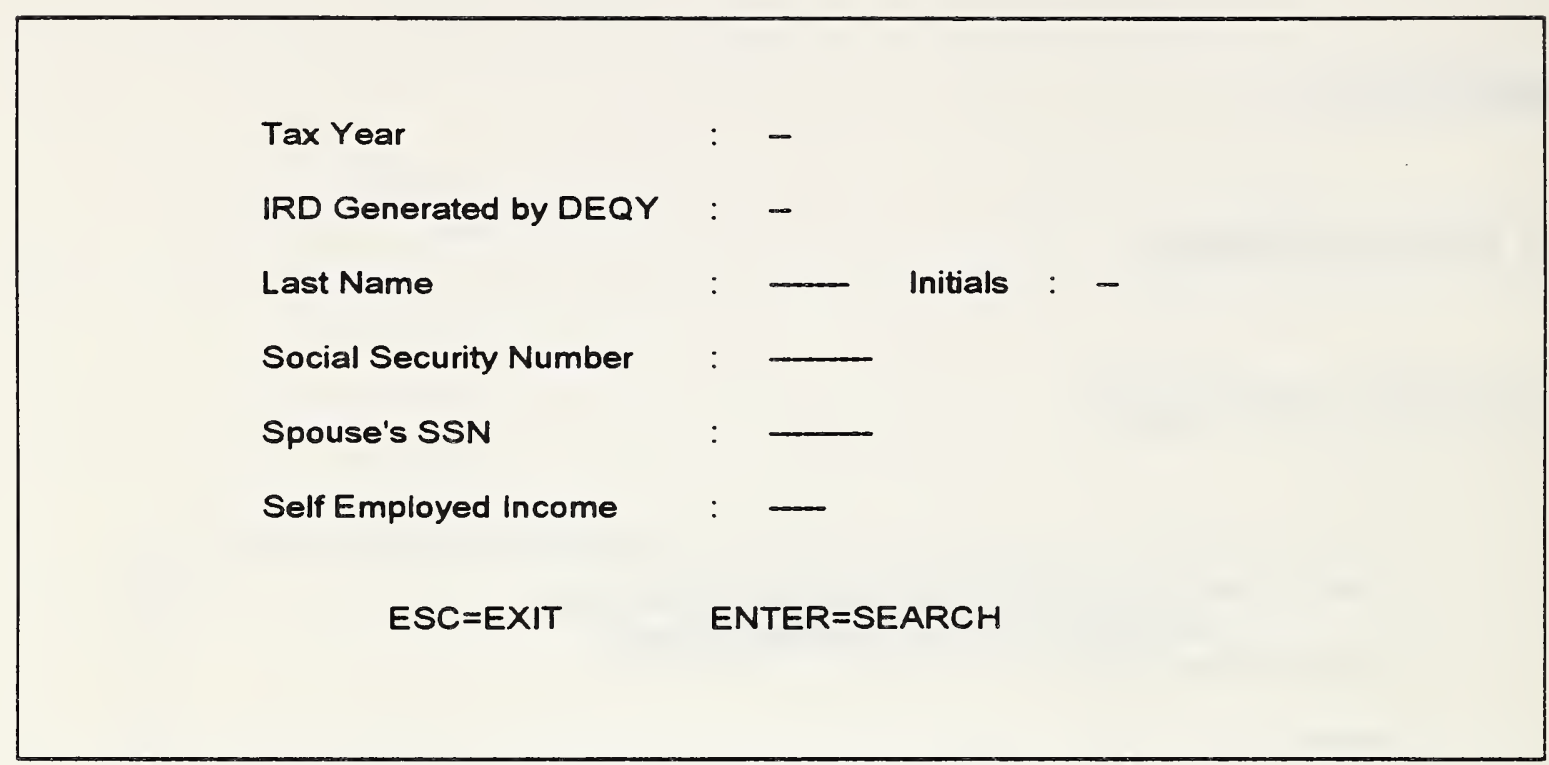

Figure A2. The query screen

Mandatory Search Parameters: Tax Year

IRD generated by the DEQY system

Last name

Optional Search Parameters: Initials

Social Security Number

Spouse's Social Security Number

Self Employed Income

Rules: A period is placed at the end of the last name field to indicate a search for an exact match of this last name. In other cases, the last name parameter is treated as a prefix, and a search is made for last names beginning with this sequence of characters.

At least four letters of the last name must be entered, unless a search for an exact match is indicated. 
In Order to ...

- Move between fields or within a field, use the Tab and Arrow keys.

- Delete a wrong character, use the Backspace key.

- View the year and IRD from the previous query, press Pg Up.

- Print the information on the screen, press Print Scrn.

- Exit the program, press Esc.

- Retrieve matches, press Return.

If ...

- No records match the search parameters, a message is displayed. Press Esc to return to the query screen.

- More records match the search parameters than can be reasonably retrieved, a message is displayed asking to refine the search. Press Esc to return to the query screen.

- A reasonable number of matches are found, the records are displayed on the screen for the user to browse. 


\section{A.3 The Browse Screen}

\begin{tabular}{|c|c|c|c|c|c|c|}
\hline \multirow{3}{*}{$\begin{array}{l}\text { Name } \\
\text { AANESTA }\end{array}$} & \multicolumn{2}{|c|}{ Tax Year: 88} & \multicolumn{4}{|c|}{ IRD Generated by DEQY: 14} \\
\hline & \multicolumn{2}{|r|}{ SSN } & SEI & \multicolumn{2}{|c|}{ Spouse's SSN and SEI } & \multirow{2}{*}{$\begin{array}{l}\text { Full Name } \\
\text { ARLET AND JAMES AANE }\end{array}$} \\
\hline & AJ & 061363003 & 37692 & 564536009 & 37347 & \\
\hline ABERNAT & JF & 658724029 & 19187 & 624052003 & 31731 & JOAN AND PETER ABERN \\
\hline ACKERMA & JA & 951812019 & 34883 & 660614017 & 19187 & JOHN AND BELINDA ACK \\
\hline ADAMSON & AJ & 658724029 & 31731 & 951812019 & 36778 & AUDREY AND CARL ADAN \\
\hline ALEXAND & $\mathrm{cc}$ & 660614017 & 24776 & 256786022 & 34883 & CHARLES AND MARY ALE \\
\hline ALEXAND & $\mathrm{CF}$ & 256786022 & 35587 & 778840006 & 24776 & CYNTHIA AND ROB ALEX \\
\hline ALEXAND & ER & 778840006 & 33323 & 658724029 & 35587 & EUGENE AND ELEXIS AL \\
\hline ANDERSO & BJ & 624052003 & 36778 & 621690034 & 33323 & BEVERLY AND TERRY AN \\
\hline$E S C=Q L$ & JER & R ENTER $=1$ & TAIL SCR & PGUP/PG & 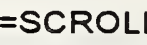 & L END/HOME $=S H I F T$ REC \\
\hline
\end{tabular}

Figure A3. The browse screen

Browse Fields (Header): Tax Year

IRD generated by the DEQY system

Browse Fields (Record): Last name

Initials

Social Security Number

Self Employed Income

Spouse's Social Security Number

Spouse's Self Employed Income 
In Order to ...

- Move between records within a page, use the Arrow keys.

- Move between pages of records, use the Pg Up and Pg Dn keys.

- View a portion of the record that is off the screen, press End.

- Return a record after viewing an off-screen portion, press Home.

- Print the information on the screen, press Print Scrn.

- Return to the Query screen, press Esc.

- View a record in detail, position the cursor to it and press Return.

\section{A.4 The Detail Screen}

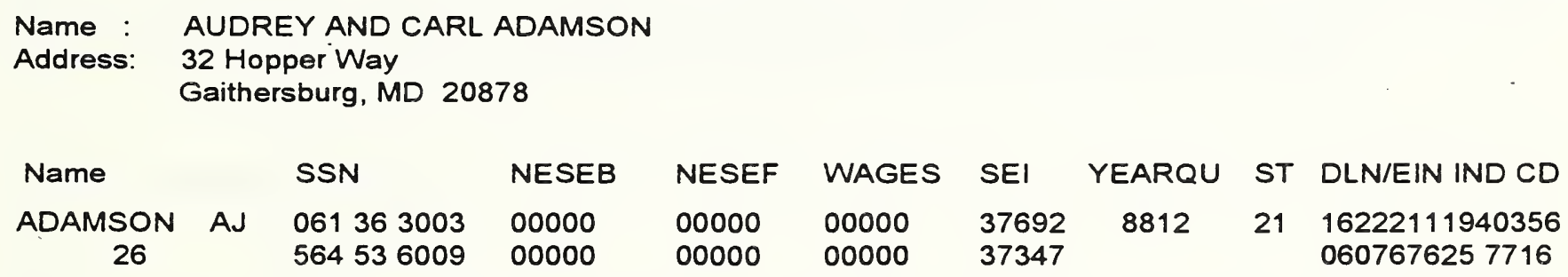

Figure A4. The detail screen

Detail Fields (Header) : $\quad$ Last name as Reported on 1040 form

Street Address

City, State and Zip Code

Detail Fields (Record): All fields as displayed on the microfilm, except the fields in the header 
In Order to ...

- Print the information on the screen, press Enter or Print Scrn.

- Return to the Browse screen, press Esc. 


\section{Appendix B}

\section{Rappôrt Setup}

\section{B.1 Terminal Parameters}

\section{USING AN RS-232 CONNECTION}

$\begin{array}{ll}\text { BAUD } & : 19200 \\ \text { DATA BITS } & : 7 \\ \text { STOP BITS } & : 1 \\ \text { PARITY } & : \text { EVEN } \\ \text { HANG UP } & : \text { NO }\end{array}$

After entering Rapport, the login prompt is displayed immediately. If it does not appear, press the Enter key. To exit, return to the login prompt and press Alt-Spacebar two times.

\section{USING A MODEM LINE}

$\begin{array}{ll}\text { BAUD } & : 19200 \\ \text { DATA BITS } & : 7 \\ \text { STOP BITS } & : 1 \\ \text { PARITY } & : \text { EVEN } \\ \text { HANG UP } & : \text { NO }\end{array}$

After entering Rapport, type: ATZ The screen displays OK. Then type: ATDP (Phone \#) for pulse lines, or ATDT (Phone \#) for tone lines. The modem dials the number, connects to the system, and the login prompt is displayed. To exit Rapport, return to the login prompt and type: CONTROL-D The computer responds with NO CARRIER. At this point, type: ATH to hang up, and the screen displays OK. Press Alt-Spacebar twice to exit Rapport. 


\title{
B.2 File Transfer Parameters
}

\author{
PORT : COM 1 or COM 2 \\ MAX. BAUD RATE : 19200 \\ BLOCK SIZE : 1024 \\ TTY BUFFER SIZE : 512 \\ RETRIES/BLOCK : 4 \\ TIMEOUT : 5
}

\section{B.3 Screen Colors}

Normal : Blue background with yellow letters

Bold : Red background with yellow letters 


\section{Appendix C}

\section{System Error Messages}

\section{C.1 User Interface Error Messages}

Too much data required for query -- Please refine query

The query entered by the user requires more than 20 files to be copied from the library, which could impact system performance due to the amount of time needed to copy and decompress that amount of files. The user must specify more letters of the last name, or enter initials upon which to search.

\section{Error! Must enter tax year, IRD and 4 letters of last name}

It is mandatory that the user enter the tax year, the IRD and at least four letters of the last name in order to request records. The user must return and provide any missing information. If the user enters less than four letters of the last name but ends the last name field with a period, this error message should not appear.

Error! Tax year must be 88 or 89

The data stored on the pilot system is only for the years of 1988 and 1989. If the user enters another year, this error message is displayed.

\section{Error! Ird XX is invalid}

The pilot system has data only for the IRD's that were found on the tapes of data sent to NIST. If the user specifies an IRD for which there is no data, this error message is displayed. 
In order to maintain management information and to keep each user's files separate, each user is assigned a user number based upon the terminal at which they are working. The terminal port is parsed into a user number, and if the resulting number is not in the range of the acceptable users, this error message is displayed.

Error opening database

Error preparing the query id

Error declaring the scroll cursor

Error opening the query

These error messages are displayed when the Informix ESQL/C database engine encounters problems with the query. Consult the ESQL/C manual for more documentation.

\section{Cannot communicate with the Handler -- Please alert Computer room}

The user interface requests cartridges from the optical disk library by sending a message to the cartridge handler device driver. If an error is returned by a messaging function, this error is displayed. This error could be cause by the handler program not being active on the file server.

\section{C.2 Cartridge Handler Device Driver Error Messages}

\section{Error getting queue}

This error message is displayed if the cartridge handler device driver could not allocate a message queue to allow communication between the user interface and the cartridge handler device driver. 
Error sending acknowledge message to user

This error message is displayed if the cartridge handler device driver receives an error when trying to acknowledge to the user interface that the cartridge requested is now mounted and available.

Jukebox error occurred while moving the disk

This error message is displayed if the ioctl() call to the library cartridge handler returns an error. 
Appendix D

Listing of the Code 


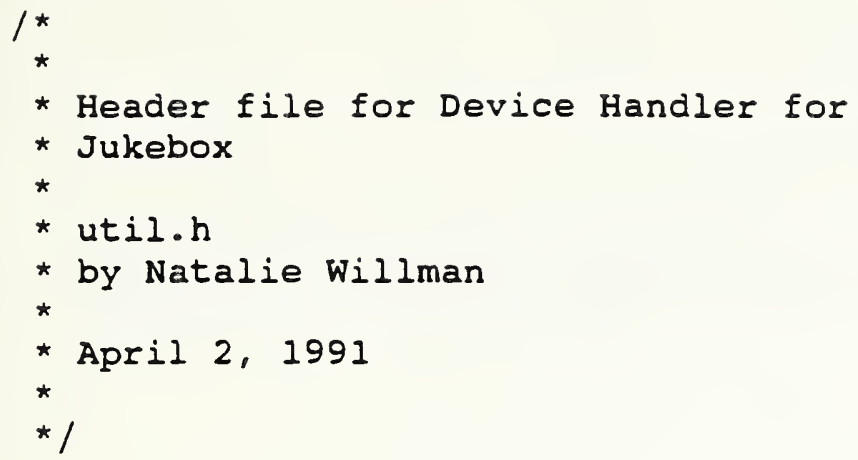

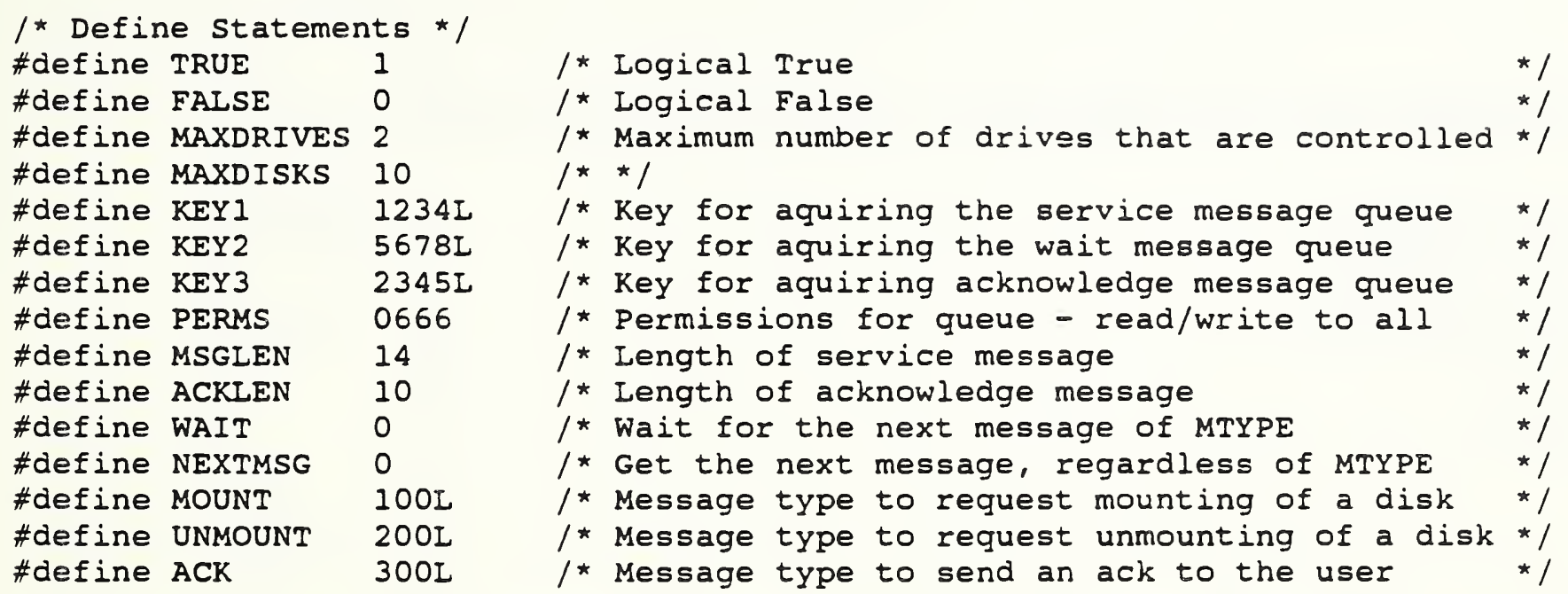

/* Structure to hold information passed to and returned from */

/* an ioctl() call to the sdlp device driver (pass thru mode */ struct IOCTI

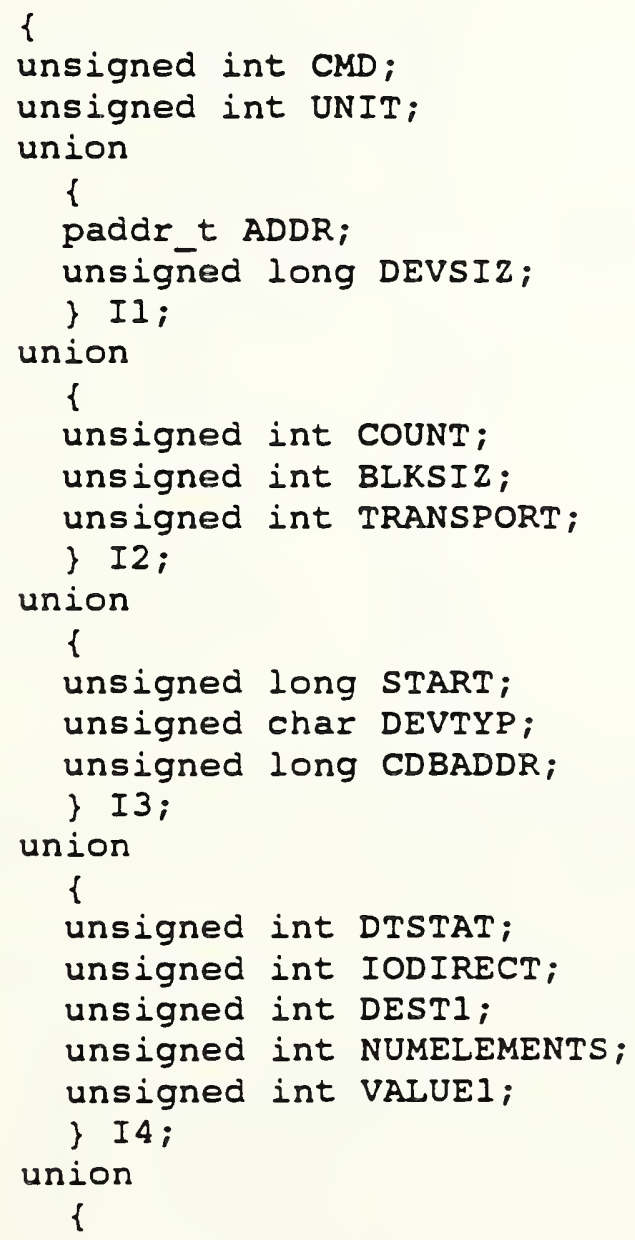




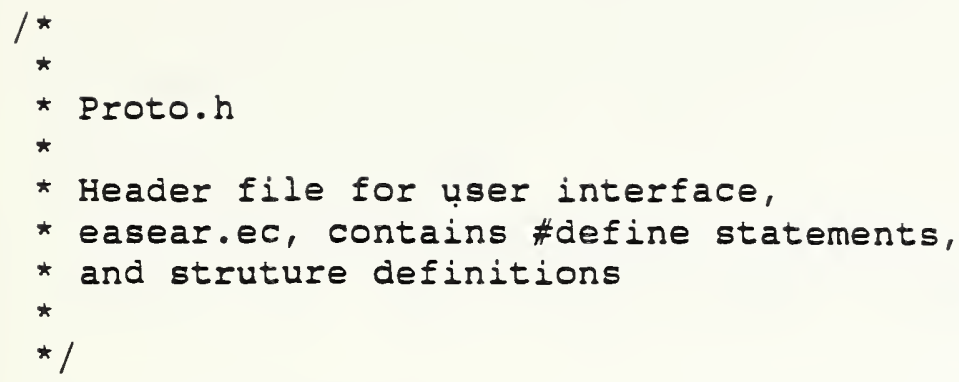

/* Global pointers to windows and panels */ WINDOW *general, *detail, *errormsg;

PANEI *pgeneral, *pdetail, *perrormsg;

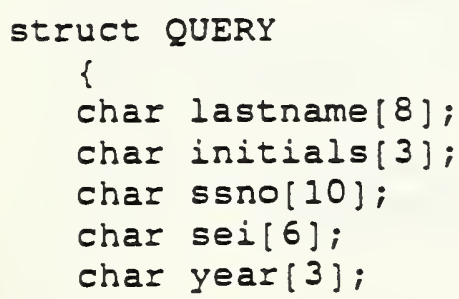




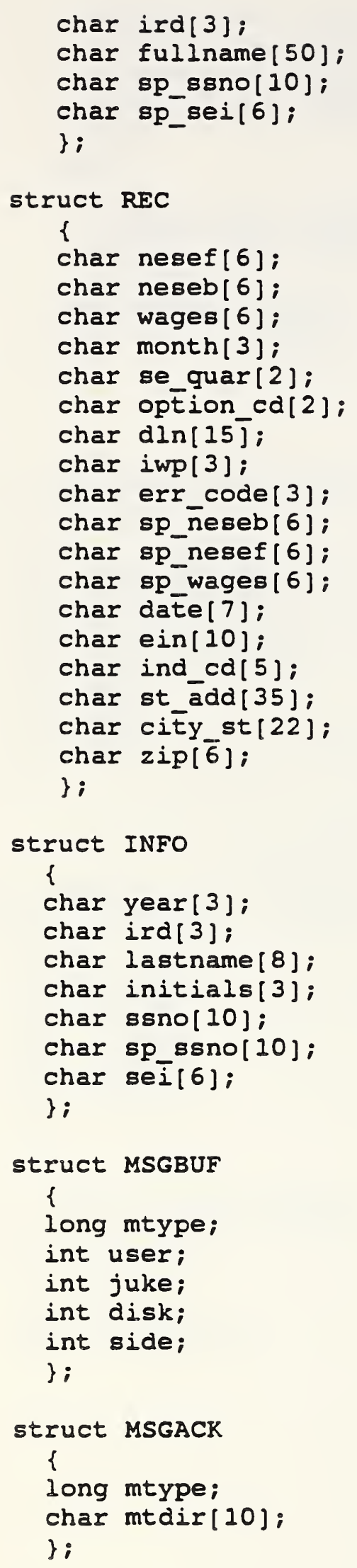




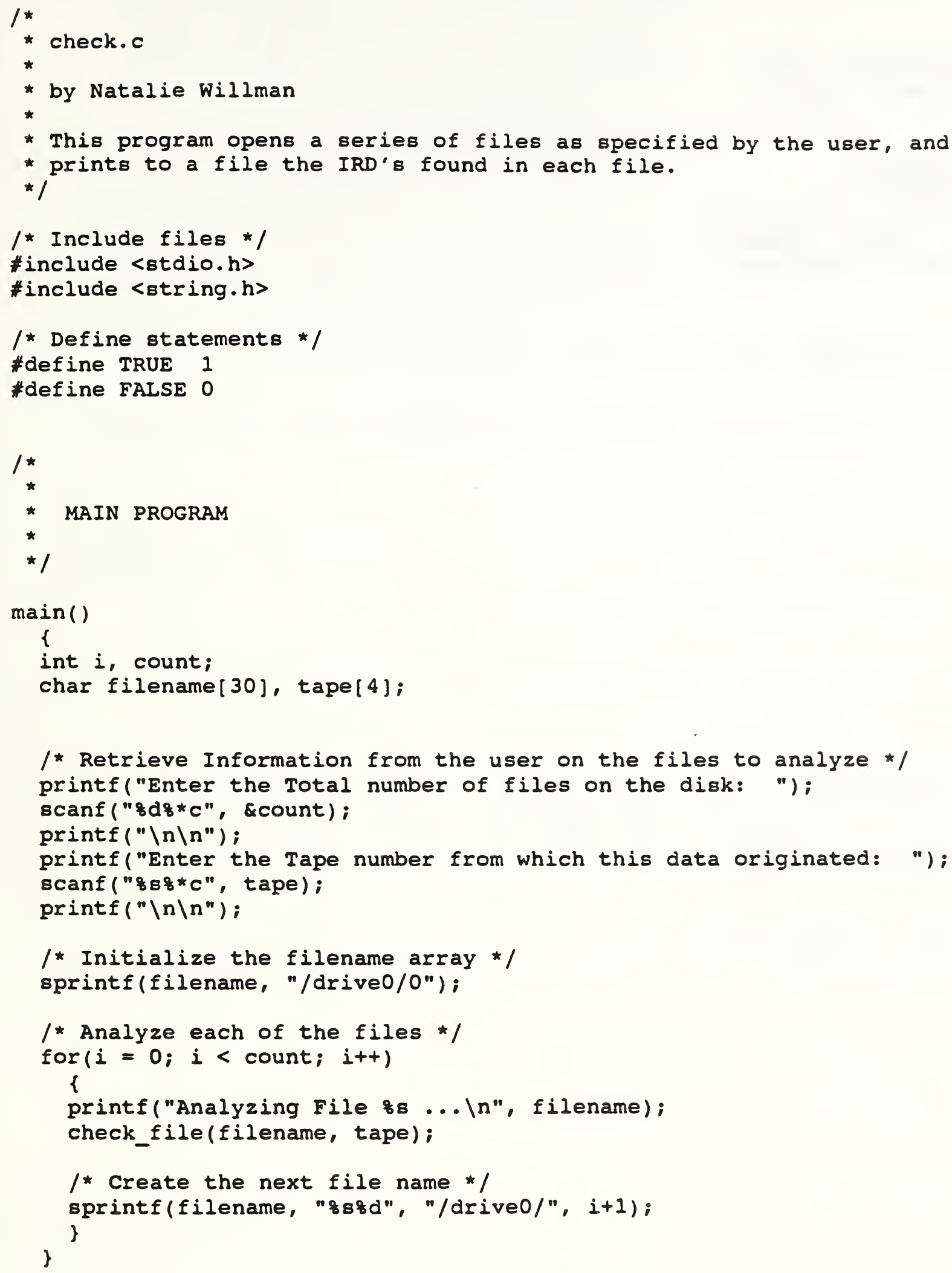


* Output: A file containing any changes in IRD

*

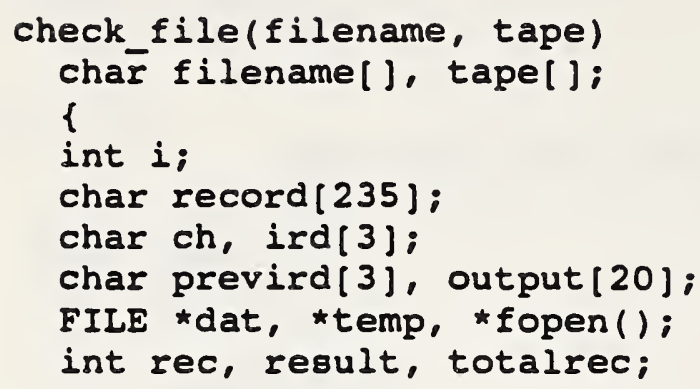
do

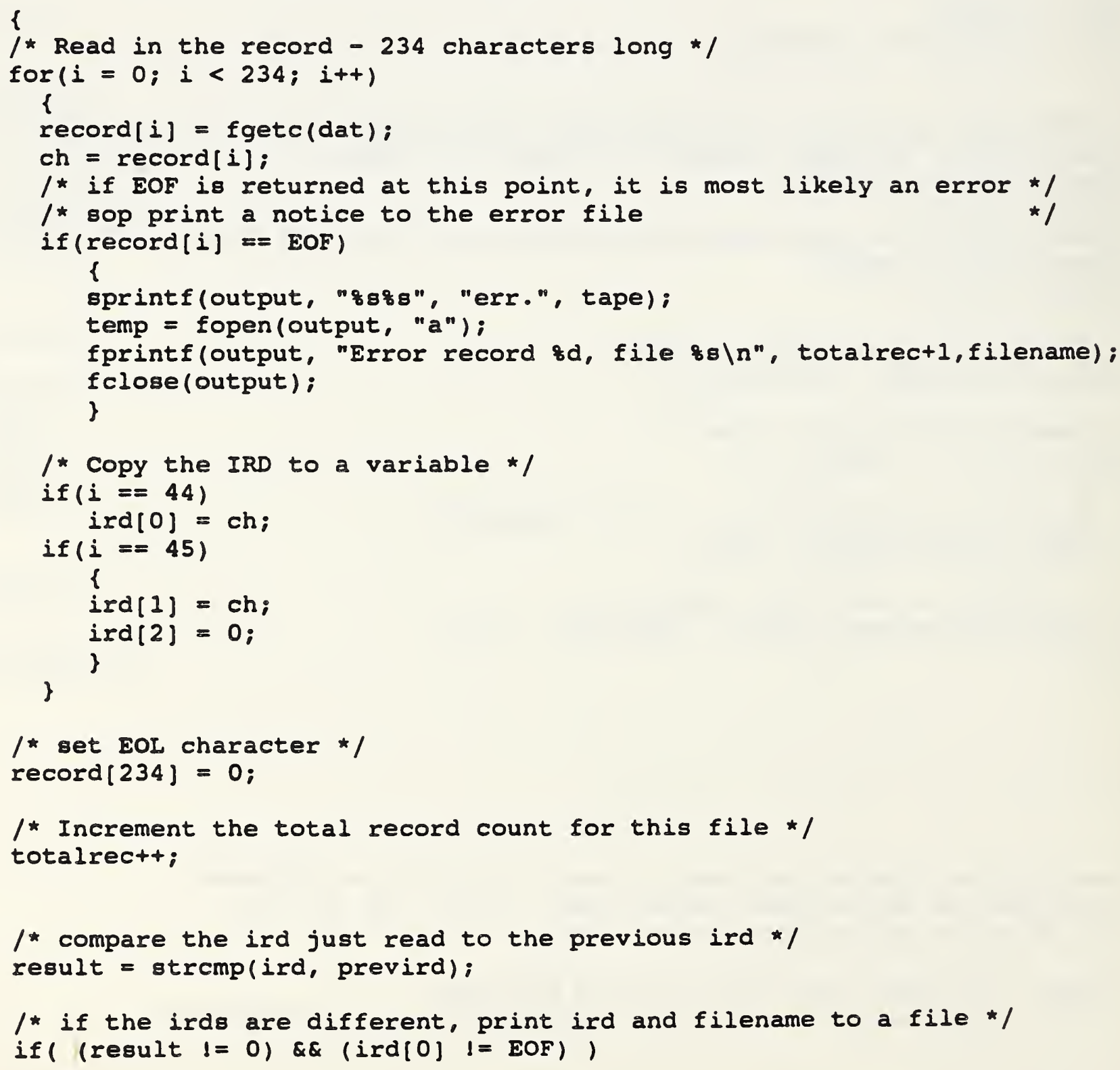




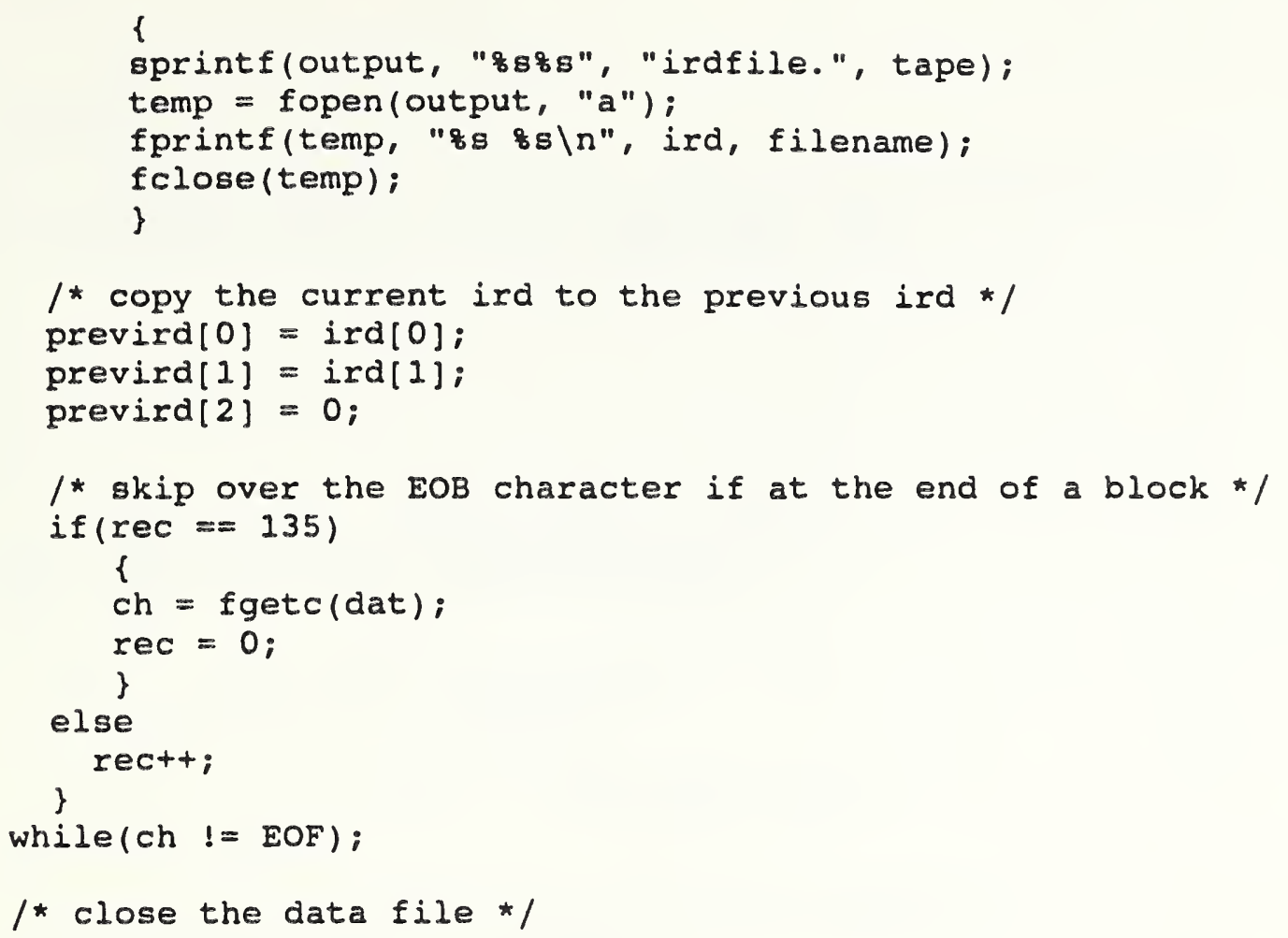





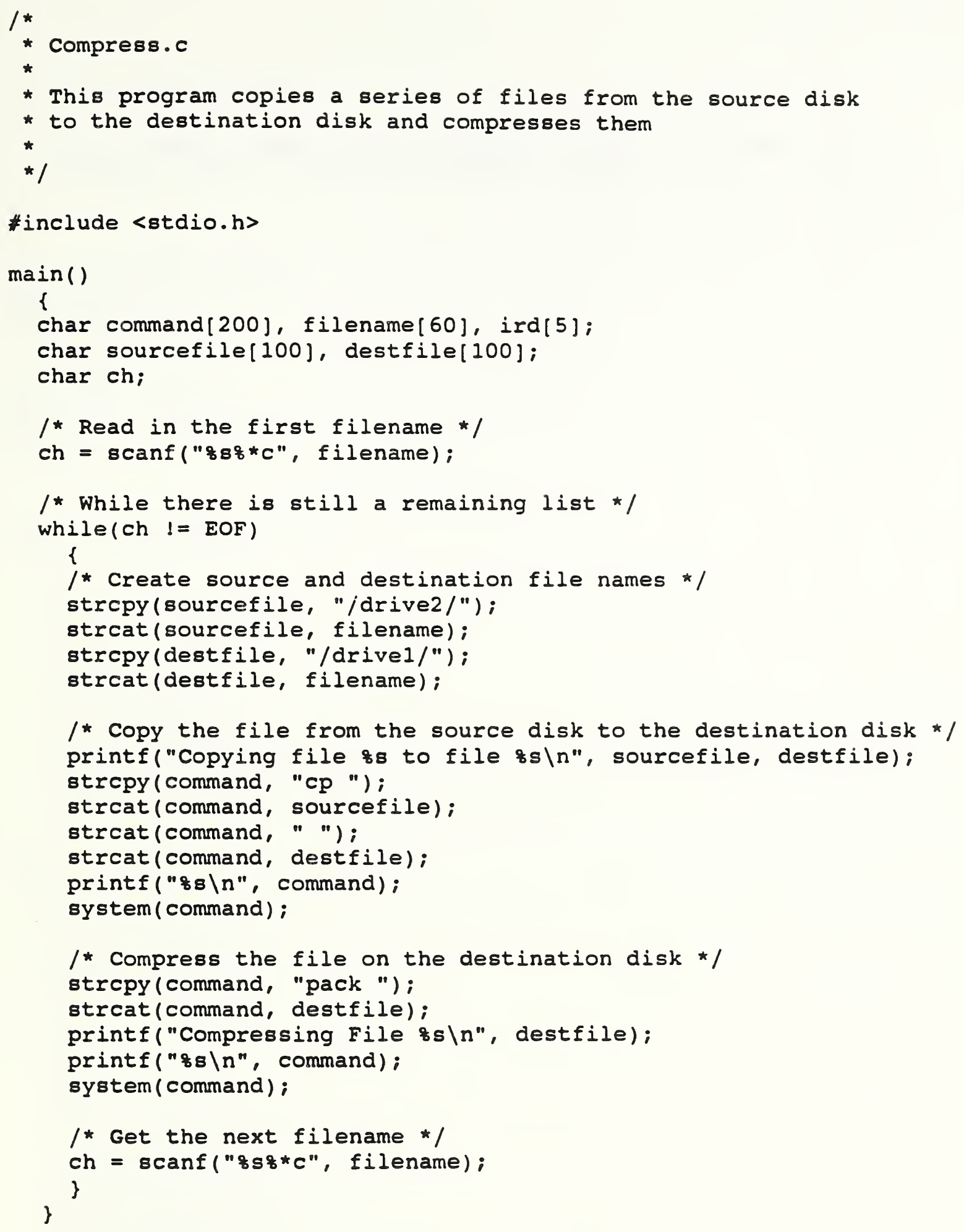





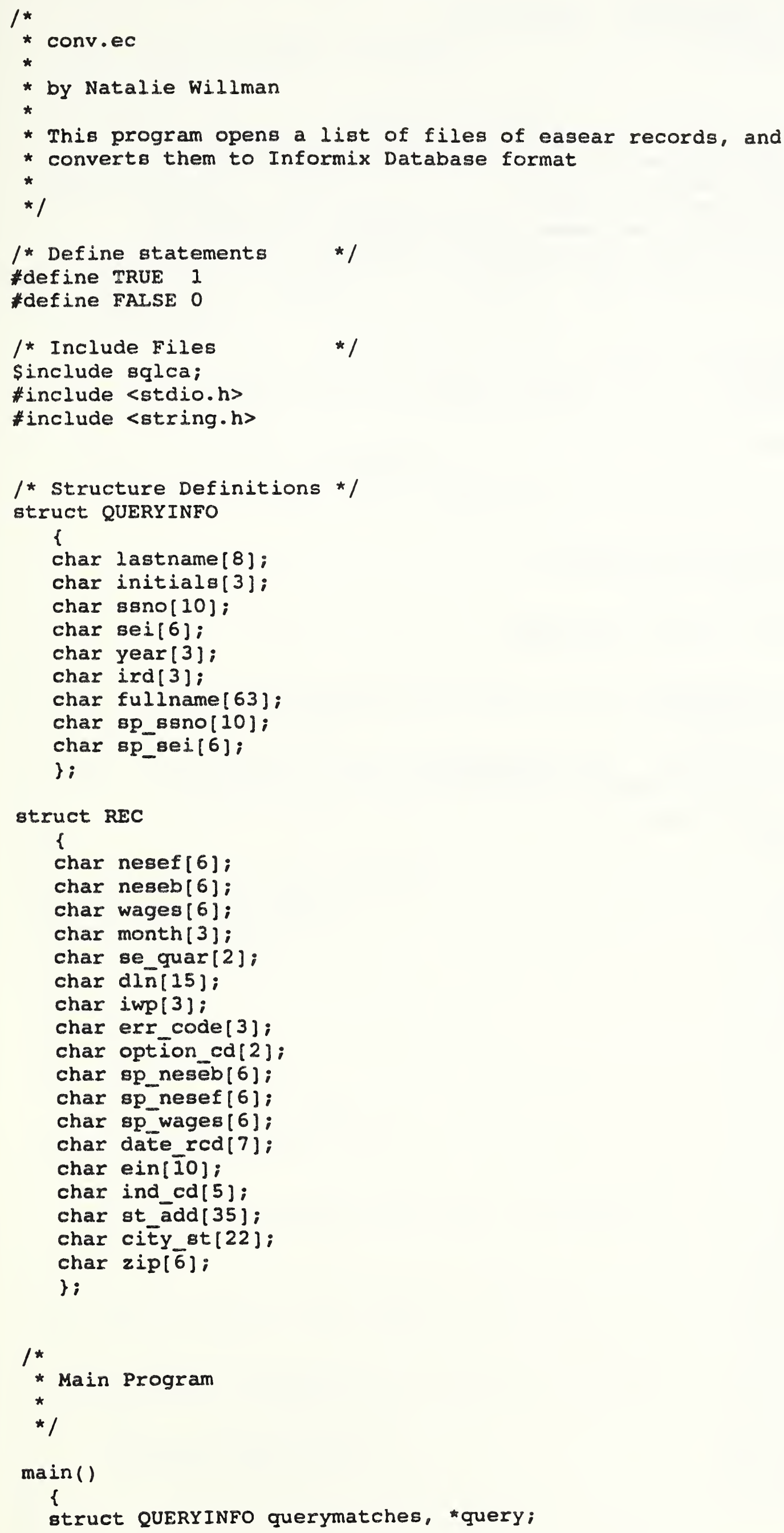


struct REC full record, *record;

char filename $[3 \overline{0}]$, cpyname[10], command[300];

FILE *namefile, *fopen();

char ch;

printf("Make sure that the source disk is mounted as read only in $\backslash n$ "); printf("/drive2, and that the destination disk is mounted as $\backslash n$ "); printf("read/write in /drivel. $|n| n ")$;

printf("Make sure that you have done $a$ ' $1 s^{\prime}$ of the source disk $\backslash n$ "); printf("and stored the file as 'names' in the current directory $\backslash n$ ");

printf("Press any key to continue ... $\backslash n \backslash n$ ");

getchar ():

$/$ * Set up pointers * $/$

record $=$ afull_record;

query = \&querymatches;

/* Open the database */

\$ database junk;

if (sqlca.sqleode)

printf ("ERROR $\backslash n$ "):

/* Open the list of files to convert */

namefile = fopen ("names", "r");

$c h=$ fscanf(namefile, "8s8*c", cpyname);

while(ch $!=$ EOF)

\{

strcpy (filename, "/drive2/");

strcat (filename, cpyname);

printf("Converting file os ... |n", filename);

/* Create the Database Table */

\$ create table test

1

lastname char(7),

initials char(2),

ssn char (9),

neseb char(5),

nesef char(5),

wages char(5),

sei char(5),

year char (2),

month char(2),

se quar char(1),

option_cd char(1).

ird char(2),

din char(14),

full name char(62),

iwp char(2),

err_code char(2),

sp_ssn char(9),

sp_neseb char(5),

sp_nesef char(5),

sp_wages. char (5),

sp_sei char(5),

date_rcd char(6),

ein char(9),

ind_cd char (4),

st_add char (34).

ciEy_st char(21),

zip char(5)

)i 
s create index test on test (lastname);

/ * read in and convert the records */

read_rec(query, record, filename);

/* Copy the files to an optical disk */

strcpy (command, "cp /usr/pilot/junk.dbs/test*.dat/drivel/");

strcat (command, cpyname);

strcat (command, ".dat");

system (command);

strcpy (command, "cp /usr/pilot/junk.dbs/test*.idx/drivel/");

strcat (command, cpyname);

strcat (command, ".idx");

system(command);

/* Drop the table -- if the table is not dropped, subsequent * /

/* records will be stored in reverse order

\$ drop table test;

/* Read in the next filename */

$c h=f \operatorname{scanf}($ namefile, "zs\%*c", cpyname); \}

\}

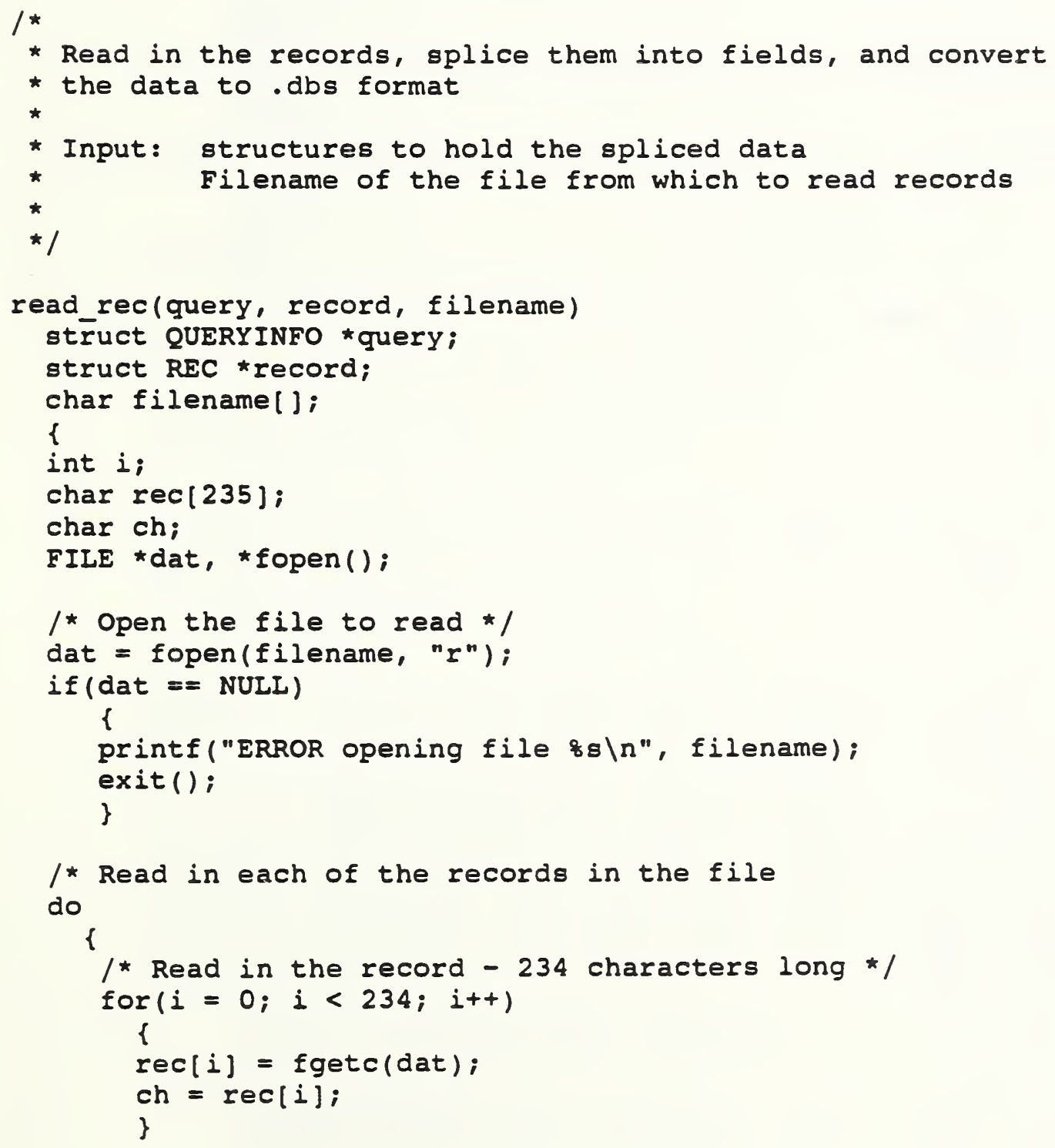




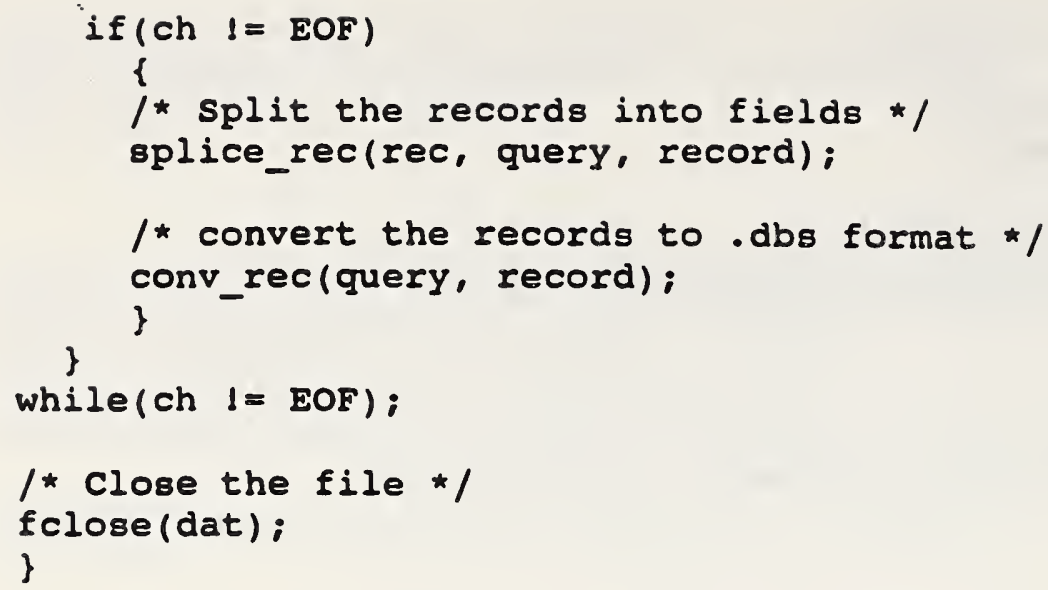


/* splice the field based on position in record string */

for $(i=0 ; i<234 ; i++)$

\{

switch(i)

\{

case 0 : case 1: case 2: case 3; case 4: case 5: case 6: query->lastname $[i]=\operatorname{rec}[i] ;$

break;

case 7: case 8 :

query->initials $[i-7]=\operatorname{rec}[i] ;$

break;

case 9: case 10: case 11: case 12: case 13:

case 14: case 15: case 16: case 17:

query->ssno $[i-9]=\operatorname{rec}[i]$;

break;

case 18: case 19: case 20: case 21: case 22:

record->neseb $[i-18]=\operatorname{rec}[i]$;

break;

case 23: case 24: case 25: case 26: case 27:

record-rnesef $[i-23]=\operatorname{rec}[i] ;$

break;

case 28: case 29: case 30: case 31: case 32:

record->wages $[i-28]=\operatorname{rec}[i] ;$

break;

case 33: case $34:$ case $35:$ case $36:$ case 37 : query->sei[i-33] = rec $[i]$;

break;

case 38: case 39:

query->year $[i-38]=\operatorname{rec}[i] ;$

break;

case 40: case 41:

record->month $[i-40]=\operatorname{rec}[i] ;$

break;

case 42 :

record->se_quar $[i-42]=\operatorname{rec}[i] ;$

break;

case 43:

record->option_cd $[i-43]=\operatorname{rec}[i] ;$

break;

case 44: case 45:

query $\rightarrow \operatorname{ird}[i-44]=\operatorname{rec}[i] ;$

break;

case 46: case 47: case 48: case 49: case 50: case 51:

case 52: case 53: case 54: case 55: case 56: case 57:

case 58: case 59:

record $\rightarrow \operatorname{din}[i-46]=\operatorname{rec}[i] ;$

break;

case 60: case 61: case 62: case 63: case 64: case 65:

case 66: case 67: case 68: case 69: case 70: case 71:

case 72: case 73: case 74: case 75: case 76: case 77:

case 78: case 79: case 80 : case 81 : case $82:$ case $83:$

case 84: case 85: case 86: case 87: case 88: case 89:

case 90: case 91: case 92: case 93: case 94: case 95:

case 96: case 97: case 98: case 99: case 100: case 101:

case 102: case 103: case 104: case 105: case 106: case 107:

case 108: case 109: case 110: case 111: case 112: case 113:

case 114: case 115: case 116: case 117: case 118: case 119:

case 120: case 121:

query $\rightarrow$ fullname $[i-60]=\operatorname{rec}[i]$;

break;

case 122: case 123:

record->iwp $[i-122]=\operatorname{rec}[i] ;$

break; 


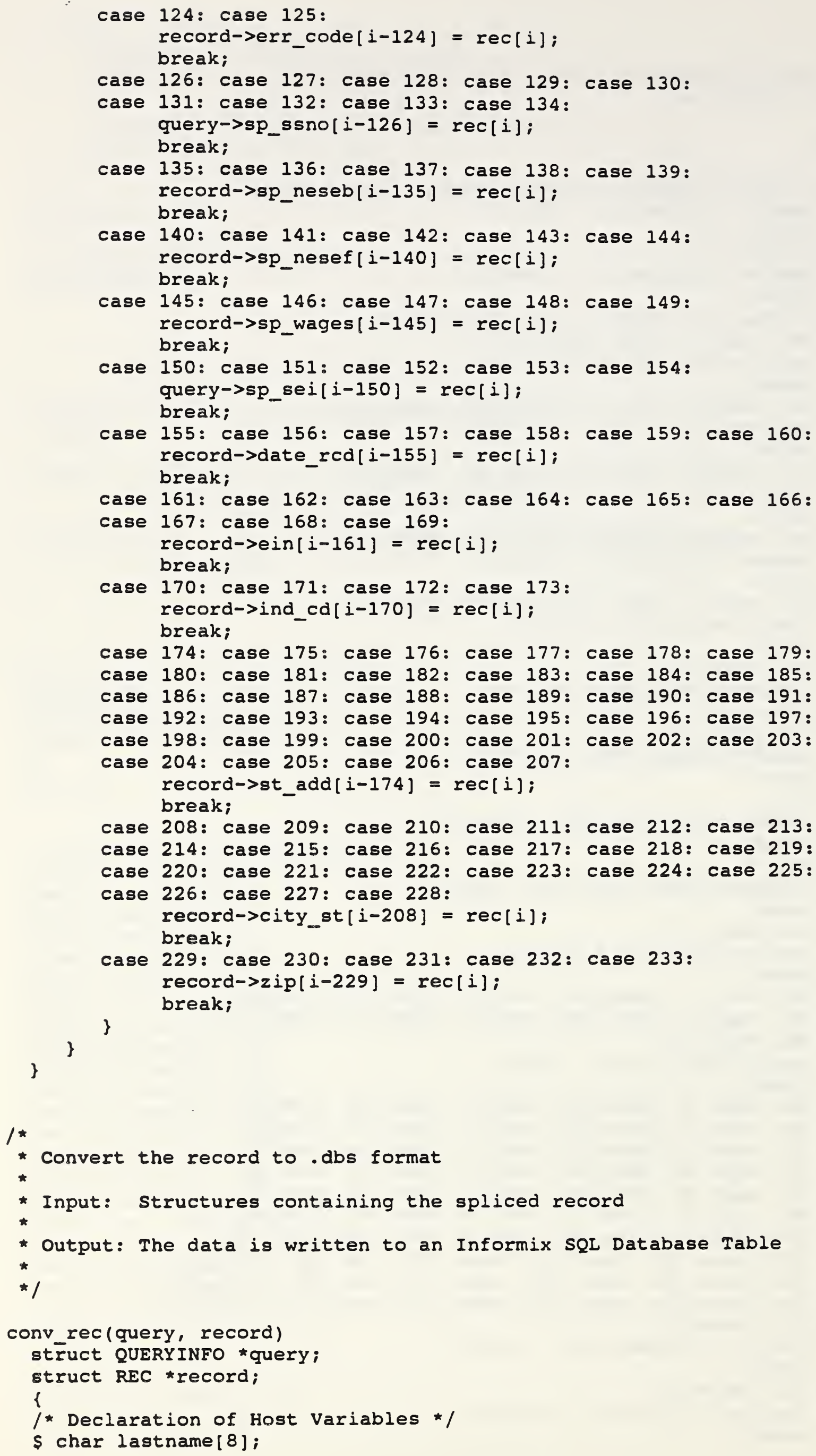



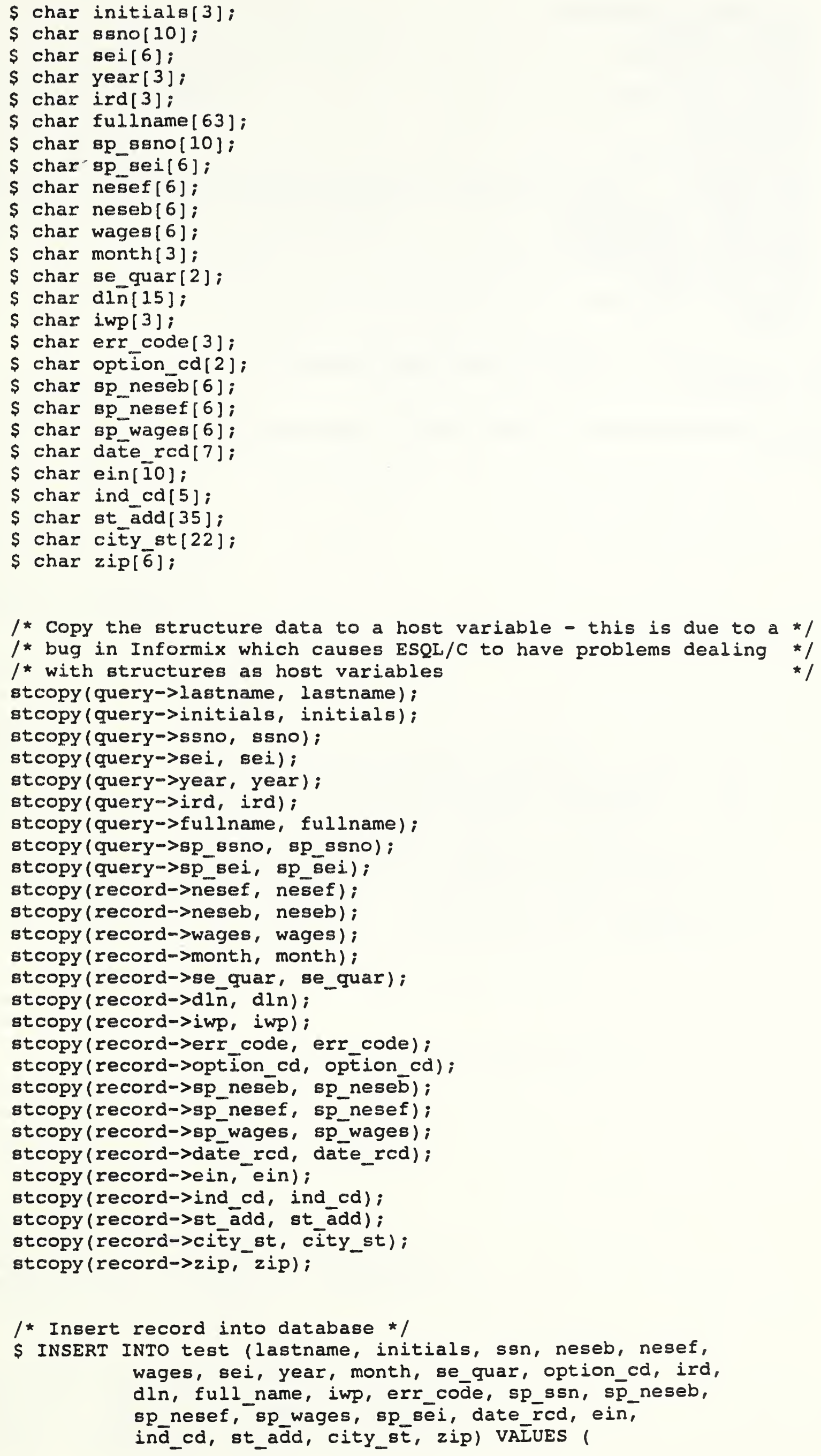
\$lastname, Sinitials,

\$ssno, \$neseb,

Sneref, \$wages,

\$sei, \$year,

Smonth, \$se_quar,

Soption cd,

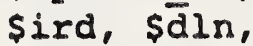

sfuliname, \$iwp,

Serr code,

\$sp_ssno, \$sp_neseb,

\$sp_nesef, \$sp_wages,

\$sp_sei, sdate_rcd,

sein, sind cd,

sst_add, sēity_st,

\$zip);

/* Check to see that no exror occured while writing to the database */ if (sqlca.sqlcode)

\{ printf("ERROR occured! - Number od $(n "$, sqlca.sqlcode); exit (): 
/* if any of the queues cannot be created, error and exit */

if $($ queueid $<0) \|$ (waitid < 0) $\|(a c k i d<0)$ )

\{

printf("error getting the queue $\backslash n$ ");

$\operatorname{exit}(0)$;

\}

do

\{

I* Check to see if both drives are currently busy - if so wait for * /

$I^{*}$ an unmount message

if ( (drives[0].busy $==$ TRUE) \&\& (drives[1].busy $==$ TRUE) ) \{

msgrev (queueid, pmessage, MSGLEN, UNMOUNT, WAIT);

print msg (pmessage);

unmtdrive = determine_drive(drives, pmessage);

empty = check_wait_queue (pmessage, waitid);

if (empty)

\{

printf("Nothing in wait queue - returning disk $\backslash n$ ");

mark idle(unmtdrive, drives);

unmt_disk(drives, unmtdrive);

ret_disk(pmessage, drives, unmtdrive); \}

else

\{

print msg (pmessage);

if (pmessage->side $==$ drives[unmtdrive].side)

\{

printf("Same side retrieved from queue $\backslash \mathrm{n}$ ");

strcpy (pmsgack->mtdir, drives [unmtdrive].mountdir);

pmsgack->mtype $=A C K+$ pmessage->user;

res = msgsnd(ackid, pmsgack, ACKLEN, IPC_NOWAIT);

if (res < 0 ) \}

printf("ERROR sending acknowledge message to user $\backslash n \% c "$, "\007');

else

\{

printf("Opposite side retrieved from queue - flipping disk $\backslash n$ ");

unmt_disk(drives, unmtdrive);

mark busy(drives, pmessage, unmtdrive);

flip disk(drives, unmtdrive);

/* Delay so that the mount will not fail due to disk not in drive */

for $(i=0 ; i<30000 ; i++)$

for $(j=0 ; j<30 ; j++)$;

mount_disk(drives, unmtdrive);

strcpy (pmsgack->mtdir, drives [unmtdrive].mountdir);

pmsgack->mtype $=A C K+$ pmessage->user;

res $=$ msgsnd (ackid, pmsgack, ACKLEN, IPC_NOWAIT);

if (res < 0 ) \}

printf("ERROR sending acknowledge message to user \n\&c", "\007');

\}

\}

else $/$ * if both drives are not busy */

\{

msgrcv (queueid, pmessage, MSGLEN, NEXTMSG, WAIT);

print_msg (pmessage);

if (pméssage->mtype $==$ UNMOUNT)

\{

unmtdrive = determine_drive(drives, pmessage);

empty = check_wait_quéue (pmessage, waitid);

if (empty)

\{ 


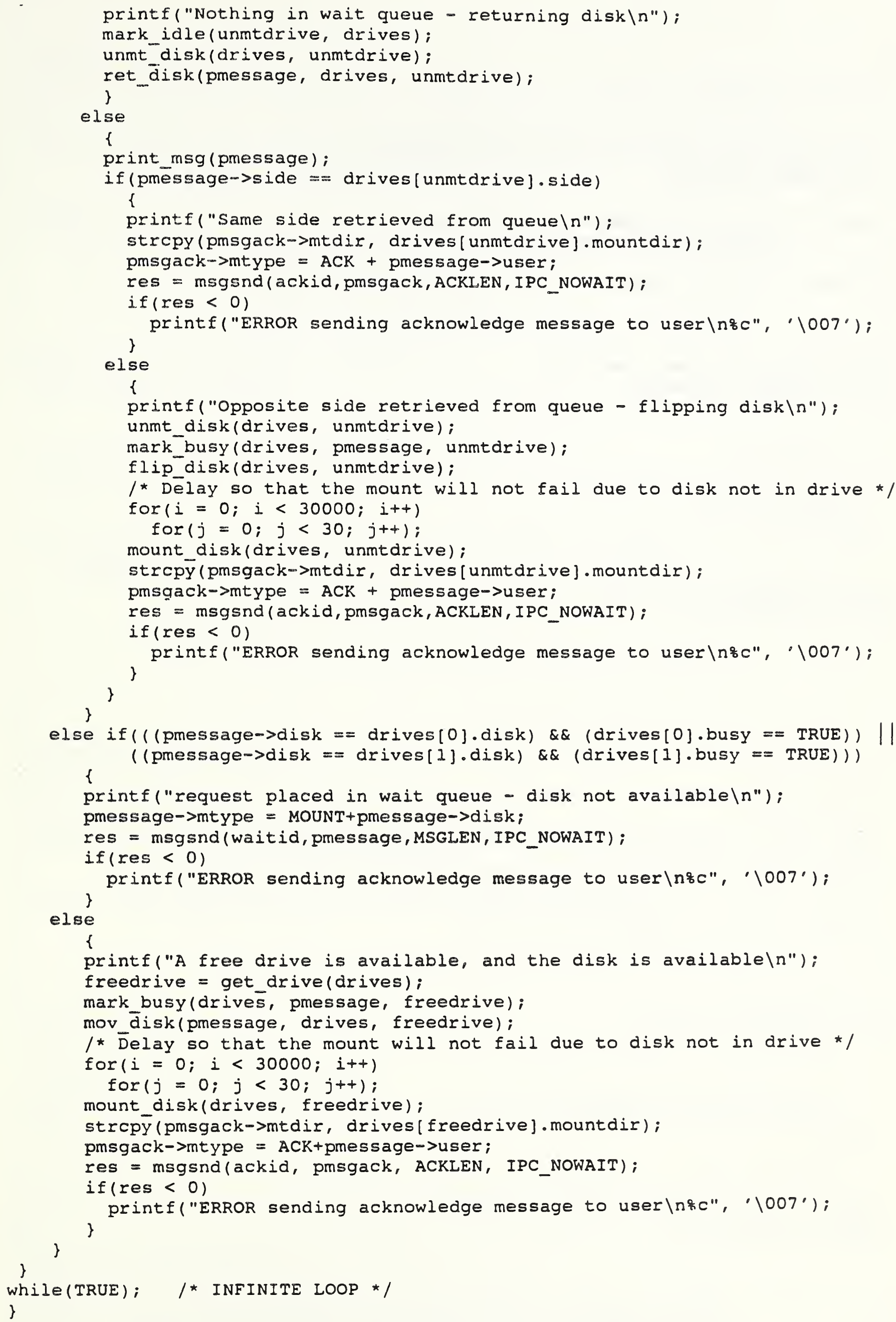




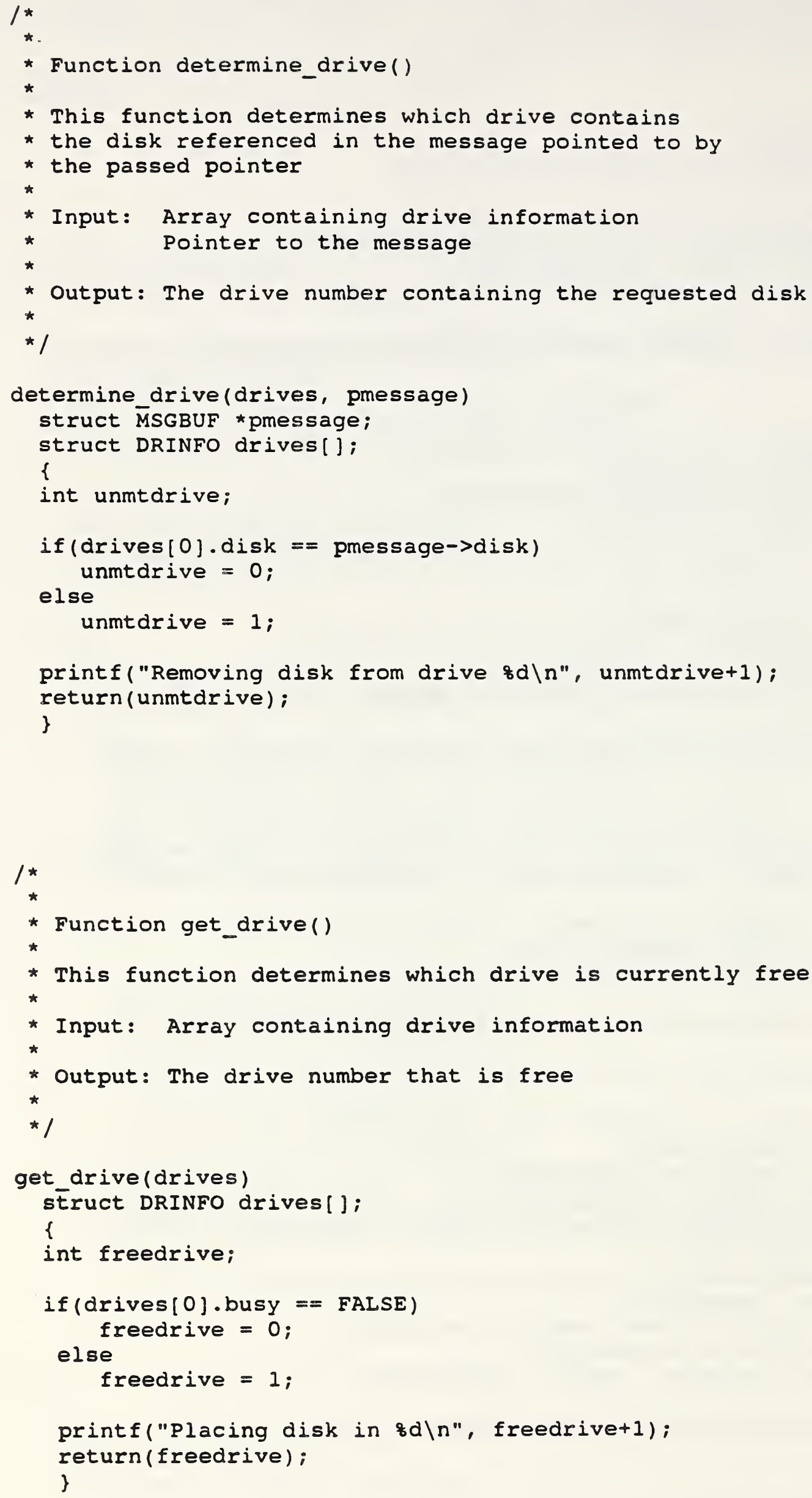




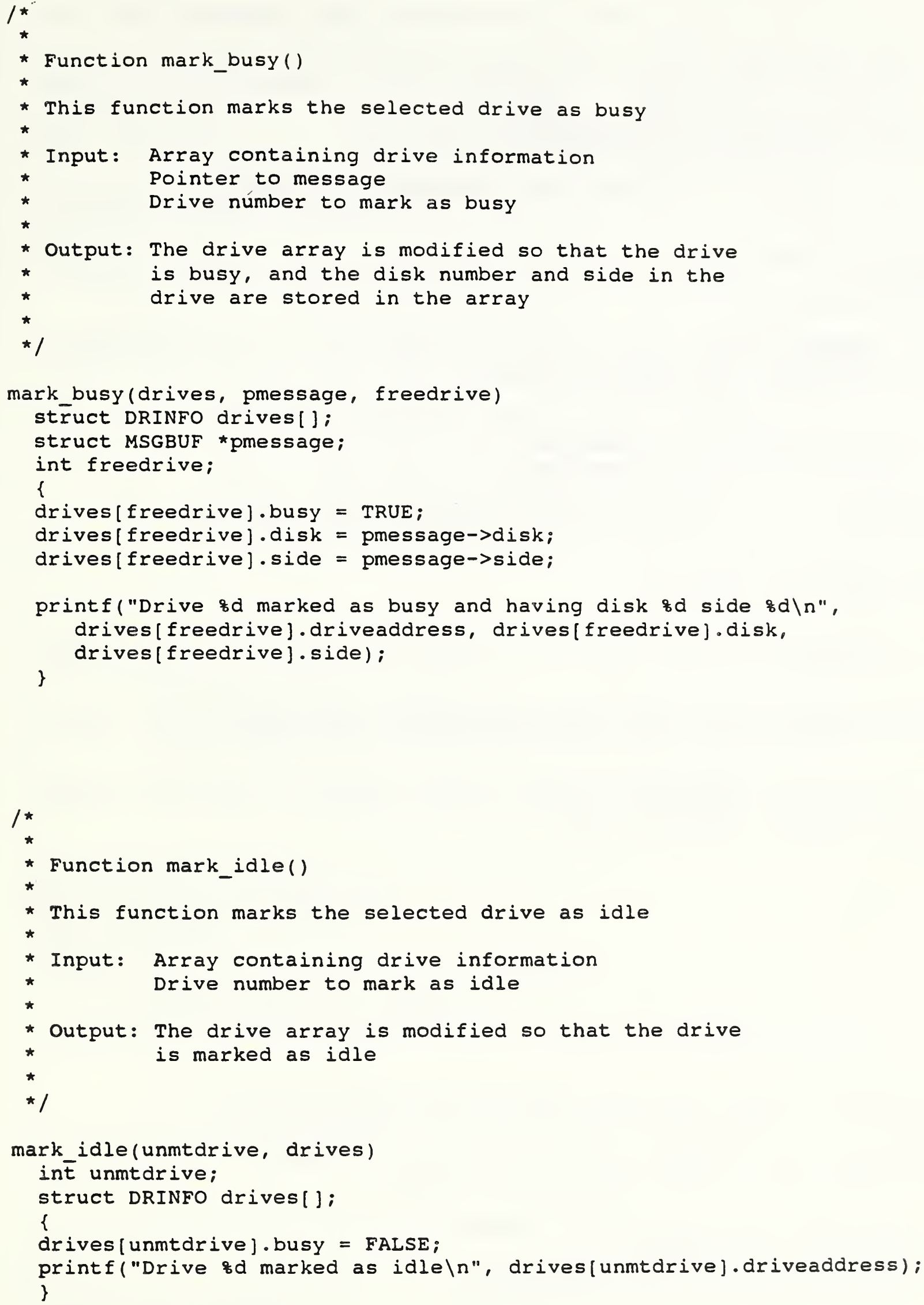




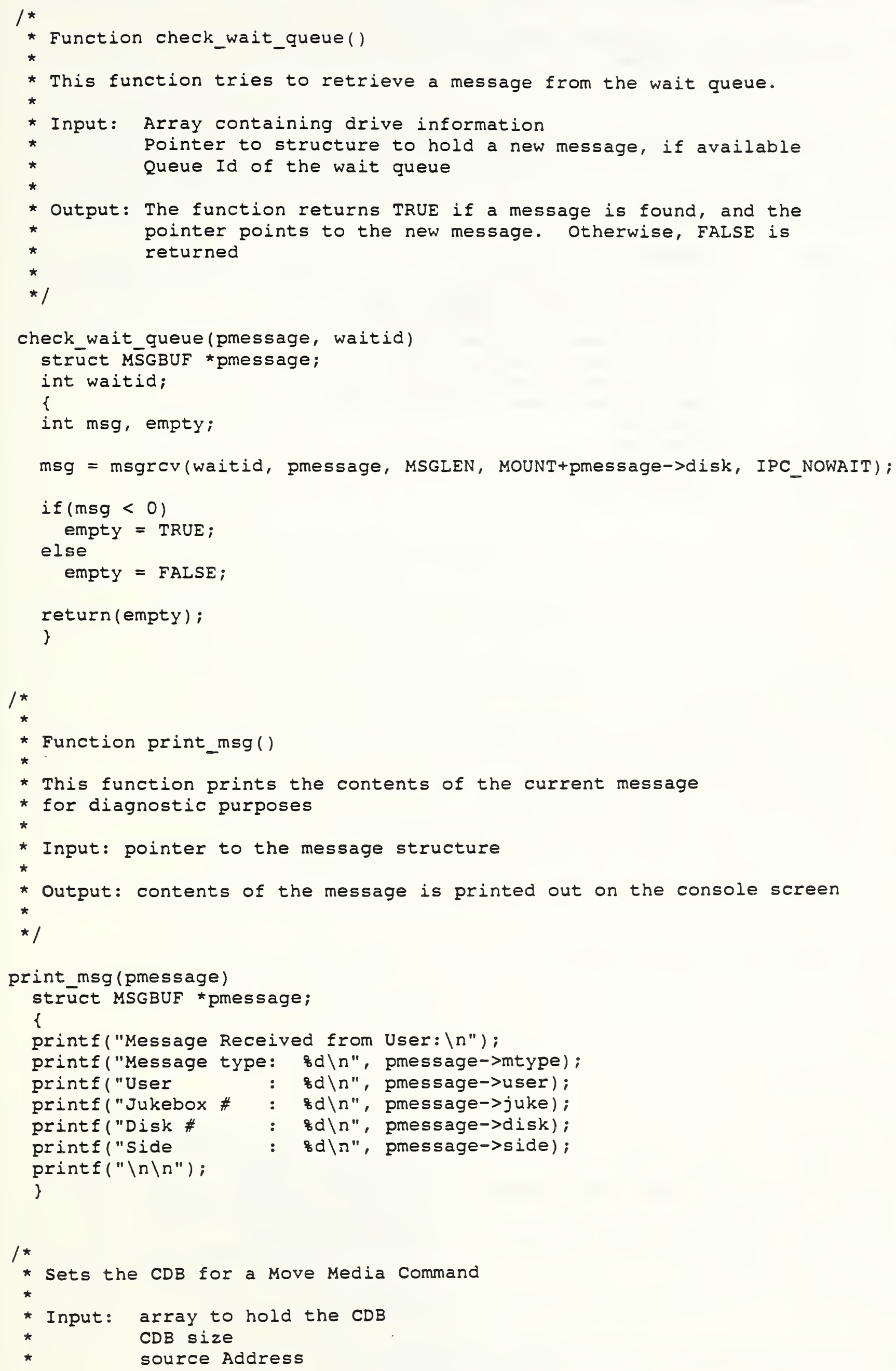



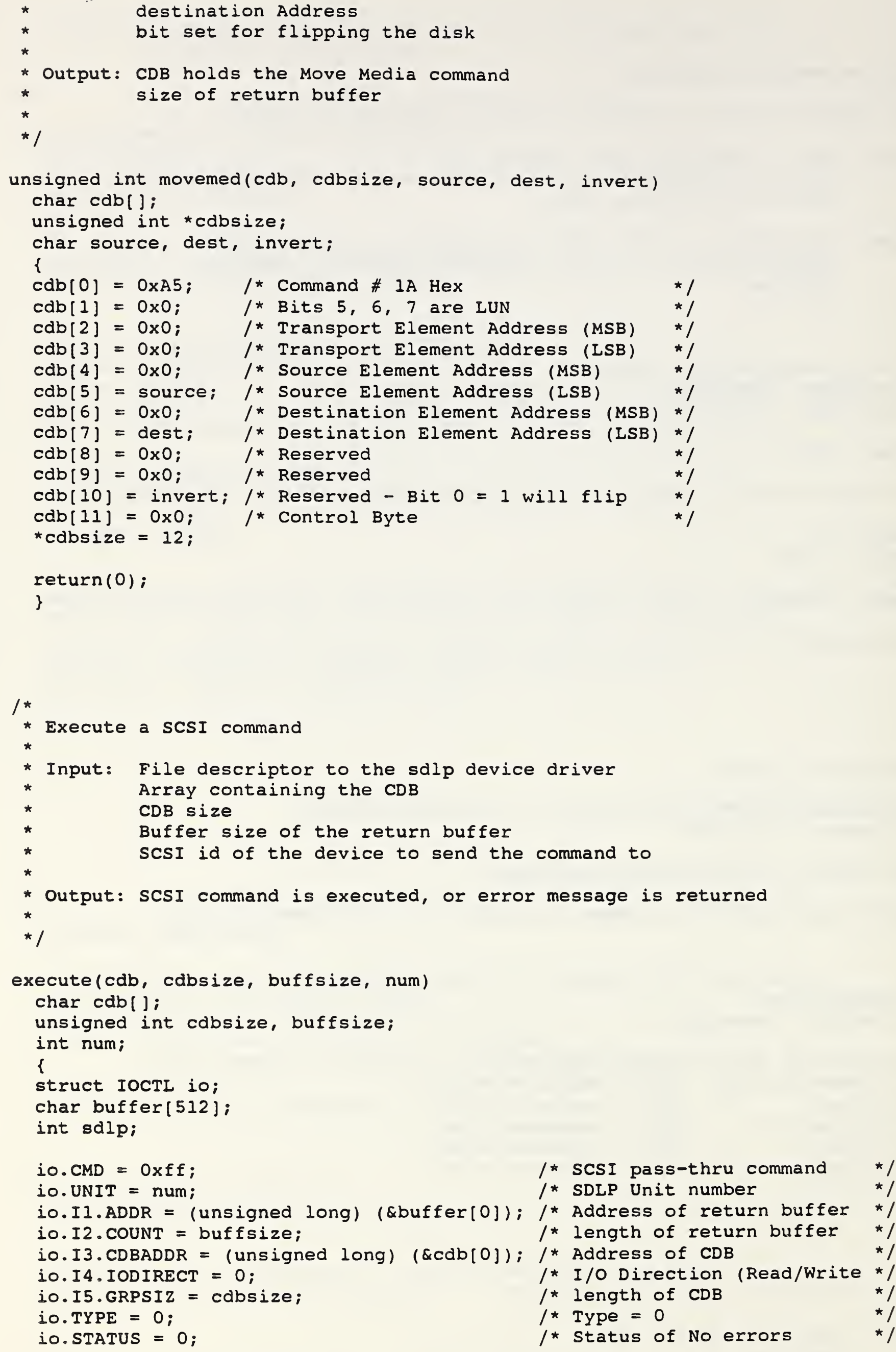


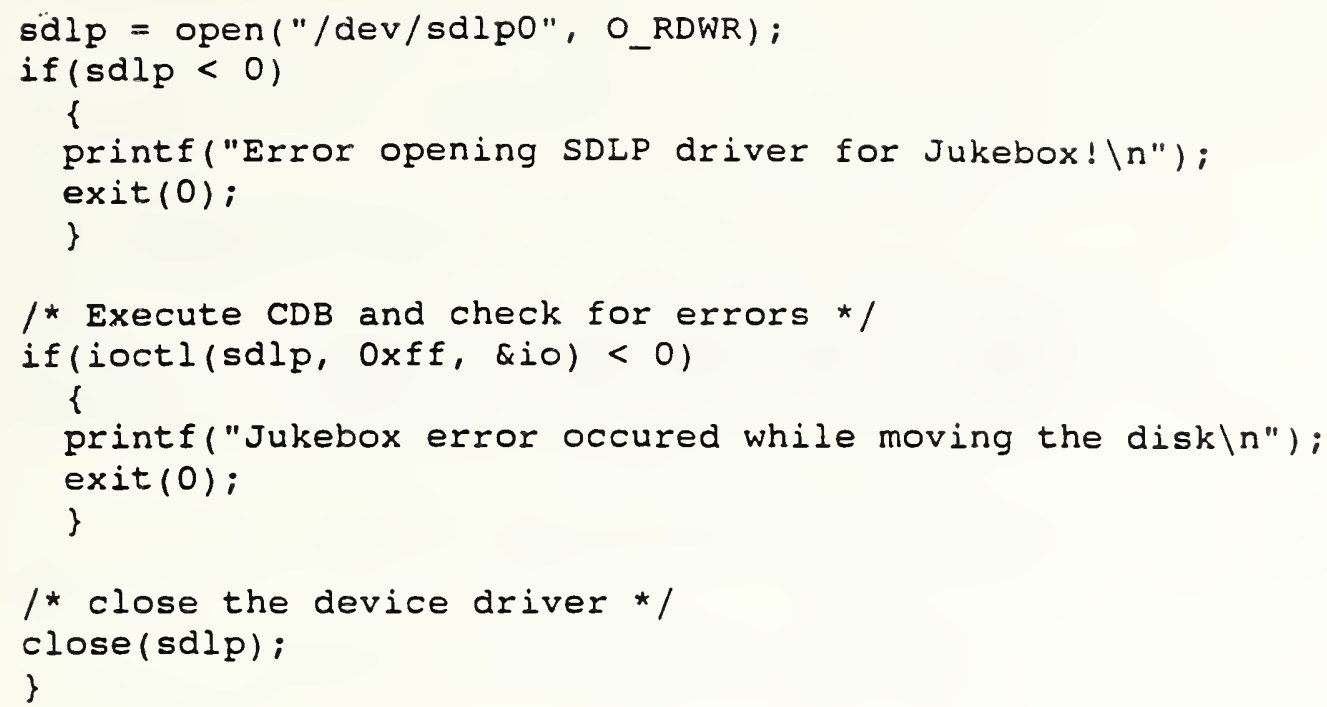





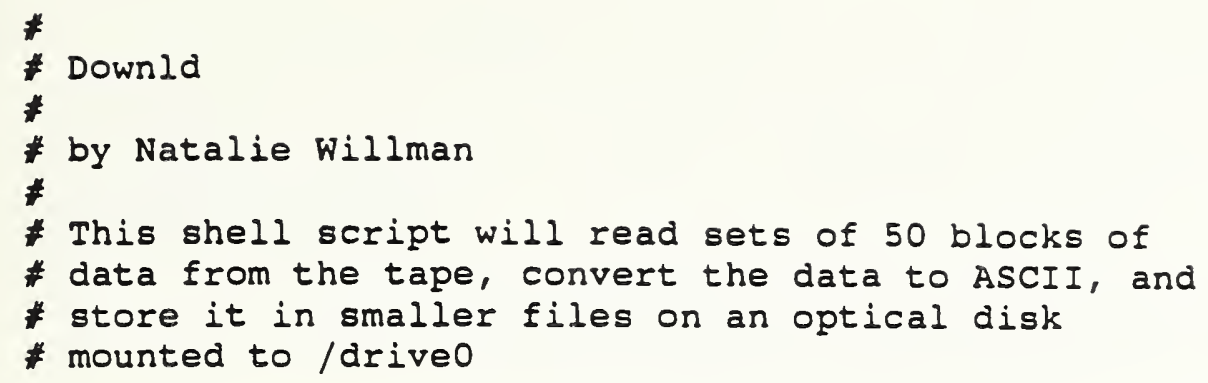

* Print the number of sets of 50 blocks entered by the user echo $\$ 1$

\# Initialize the counter and other variables counter $=0$

fuld $=\$ 1$

\# While there are still sets left, print the set number, and \# download the set, converting it to ASCII, and increment the counter while test scounter - it \$full do echo scounter dd if $=/ \mathrm{dev} /$ segNo of $=/ \mathrm{drive0} / \$$ counter $i b s=31824 \mathrm{cbs}=31824 \mathrm{conv}=a s c i i$ count $=50$ counter='expr scounter + 1 ' done

* Print the last set number (a partial set), and download and convert * the records in that set echo scounter dd if=/dev/seqno of $=/ d r i v e 0 / \$ c o u n t e r ~ i b s=31824$ cbs=31824 conveascii 



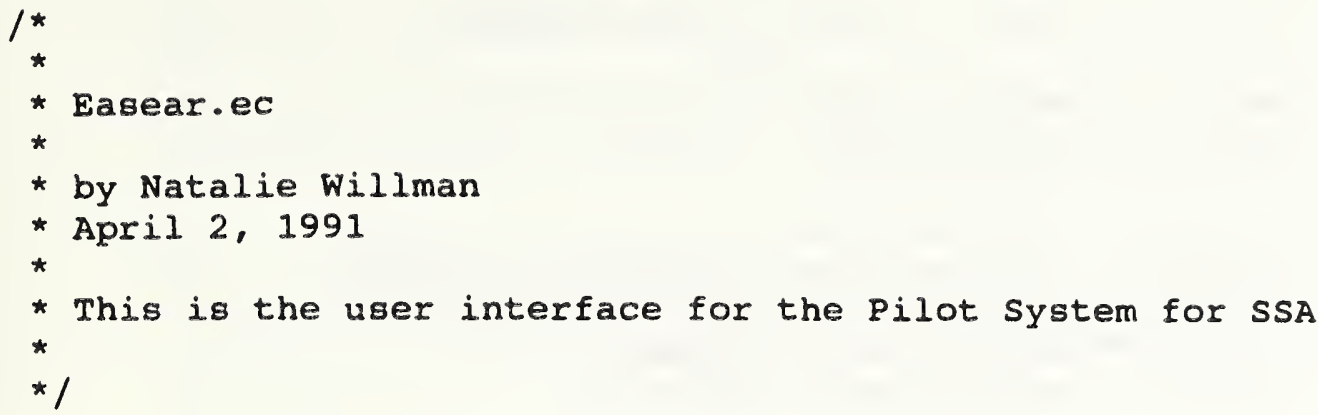

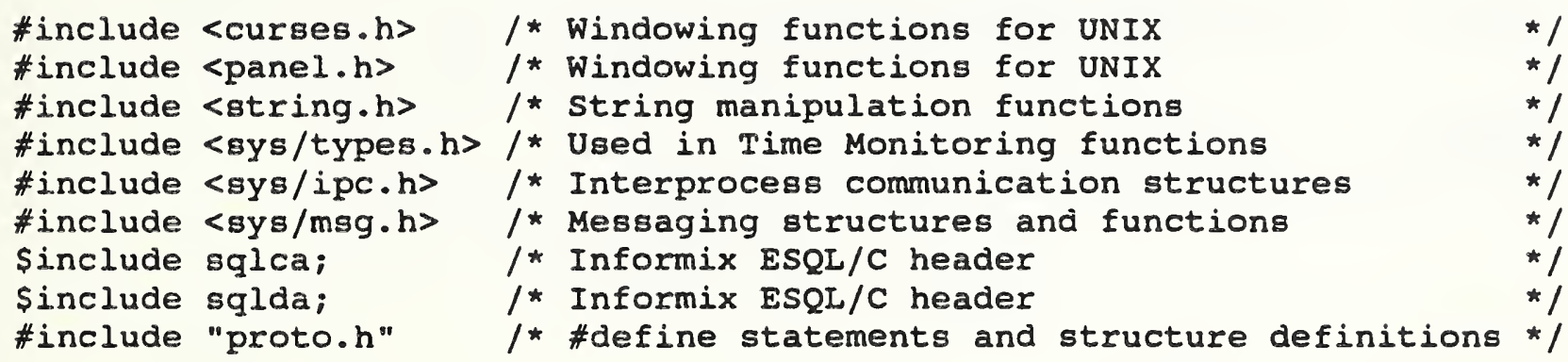

/ Host variable to receive data from the database file - this is */

$/ *$ done rather than making the true structures the host variables for */

/* two reasons: to keep control of the arrays (so they are not */

/* altered by esql, and because host variable structures cannot */

/* be arrays - bug in ESQL */

sstruct RECEIVE

\{

char lastname [8]:

char initials[3];

char ssno[10];

char nesef [6];

char neseb [6];

char wages [6];

char sei[6]:

char year [3];

char month [3];

char se_quar [2];

char option_cd[2]:

char ird[3];

char dIn[15];

char full name [50];

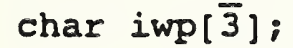

char err code[3];

char sp_ssno[10];

char sp_neseb[6]:

char sp nesef[6];

char sp wages [6];

char sp_sei[6];

char date rcvd[7];

char ein[10];

char ind cd[5];

char st add[35]:

char city st [22];

char zip [6];

) retrvar;

/* Management Information structure.

\{

long int logtime; $\quad /$ * Time that the user is logged into the system

long int logcount; $/$ * Count of logins to the system 


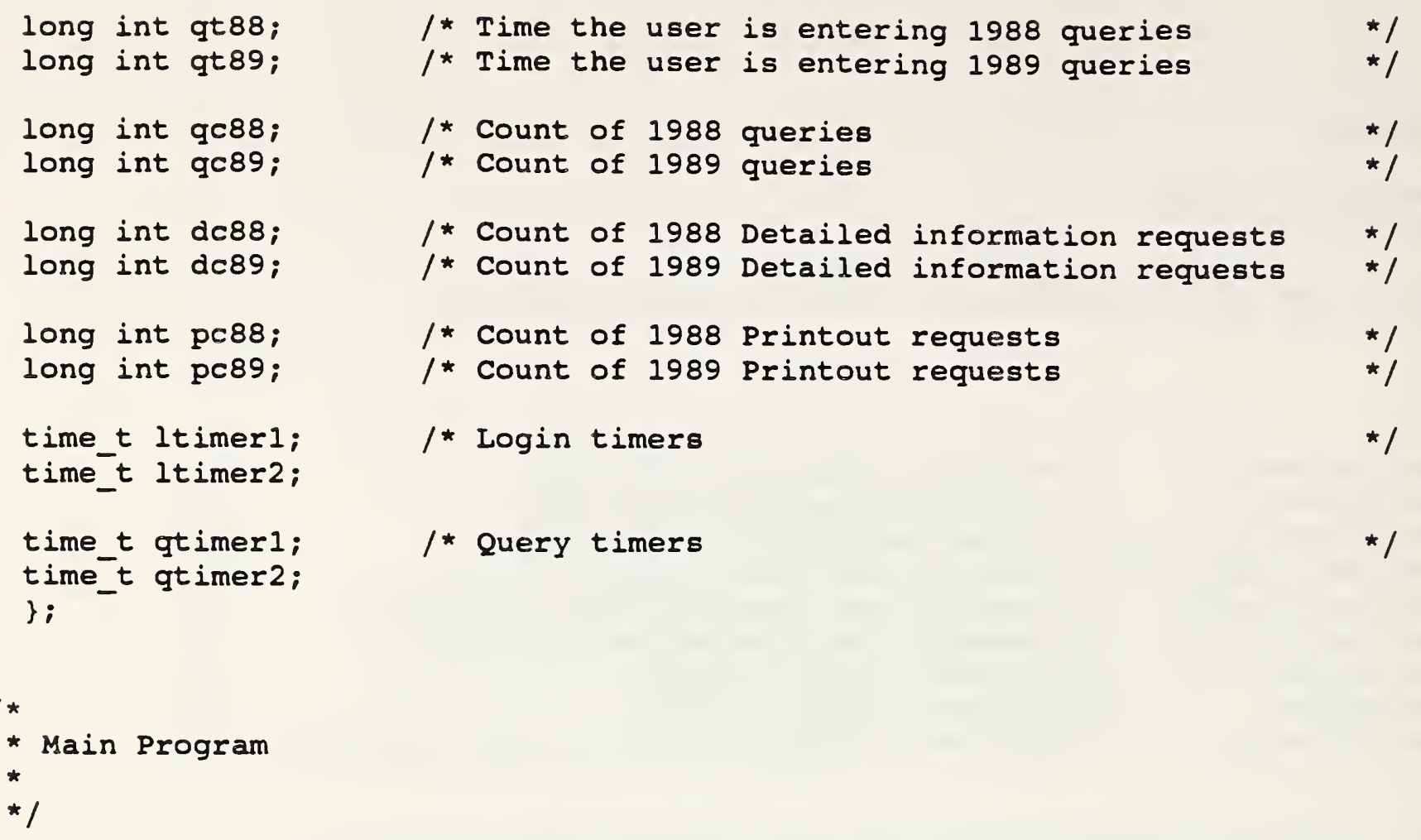

$\operatorname{main}()$

\{

struct MANAGEINFO minfo, *pminfo; $/$ * management information structure */

struct QUERY querymatches[MATCHSCR]; $/$ * contains browse info on matches */

struct REC full_record[MATCHSCR]; $/$ * contains full info on matches *

struct INFO usrquery[2]; $/$ * contains users query parameters * $/$

char qinput, binput; $\quad /$ * Individual char of user input *

char get_query(); $/$ * function to get a user's query */

int nummatches, curmatch; $\quad$ * \# of matches in memory, and *

int user; $/$ * User number - based on tty port */

int possible; $/$ * Is the user's query possible */

/* Initialize the screen to use the ETI interface and setup windows * /

/* Obtain the user number, and setup management information */

initscr ();

setup_windows ( ):

user $\equiv$ get_user_no();

pminfo = \&minfo;

init mi(pminfo);

inc_log_count (pminfo):

start_log_timer(pminfo);

clrquēry (üsrquery, TRUE);

/* Get the user's queries, until he presses ESCAPE, indicating the */

/* session is complete

do

\{

start_query_timer(pminfo):

qinput $=$ geEt query (usrquery);

if (qinput $!=-$ ESC)

\{

/* Display the wait message

*

disp_wait();

/* select matches from the database

*I

possible = select_matches(usrquery, user);

/* Remove the wait message 

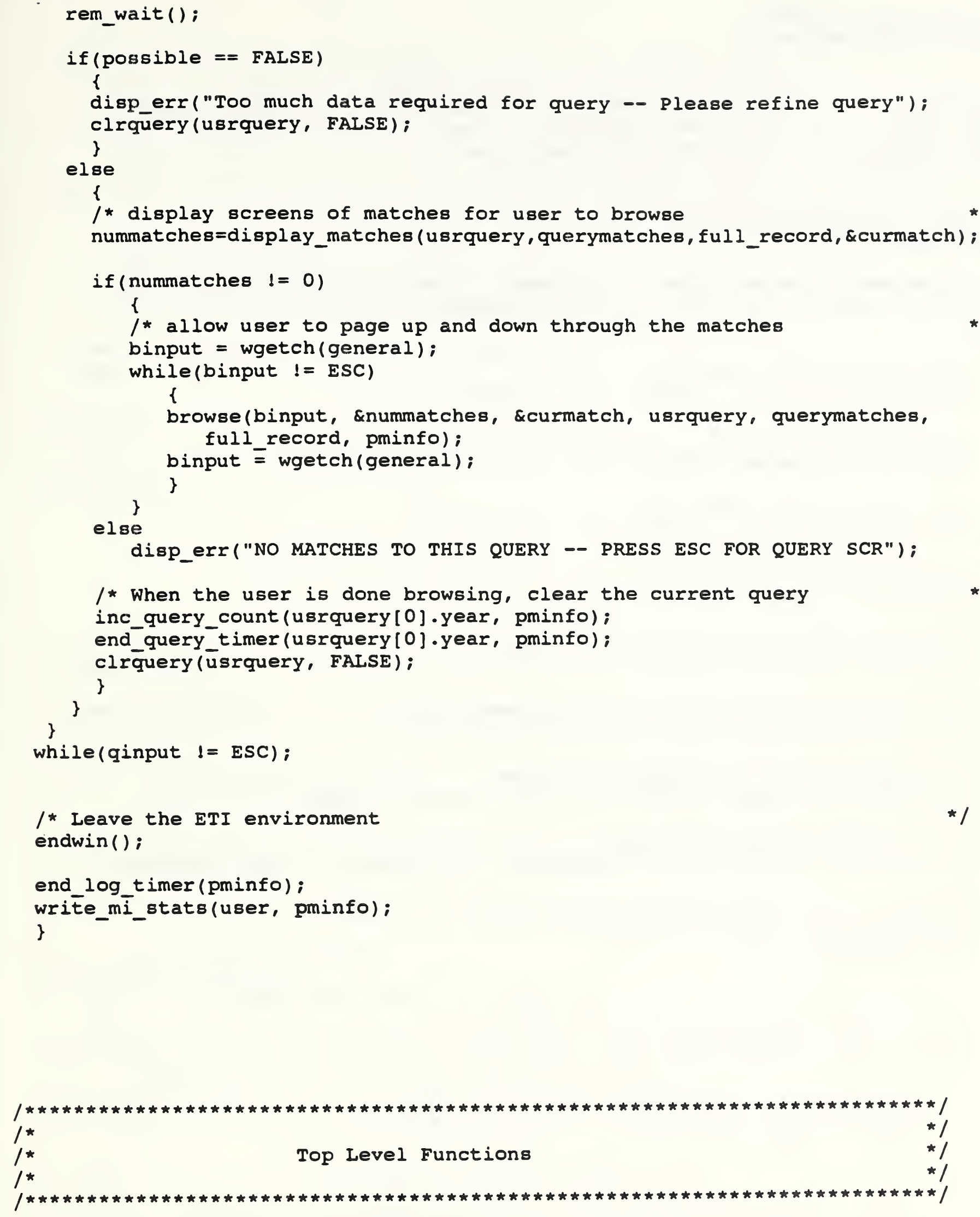

$1 *$

* This function prompts the user for the query information

* and stores it in the userquery structure

* Input: pointer to structure to hold the users query (usrquery)

* Output: The structure usrquery is filled with the search parmeters

* The indicator by the user whether to search for matches (ENTER) or quit the query session (ESC) 
char get_query (usrquery)

struct INFO usrquery[];

\{

int row, col; /* Location of cursor on the screen */

int done; $\quad /$ * boolean variable to indicate when the query is entered */

char input; $/$ * individual character of user's input */

int lastlen; $/$ * Length of last name in the query *

char errmsg[50]; /* Error message to print */

/* Clear the background window */

clrWIn (GENERALWIN) ;

/* Allow user to enter a query and check to make sure that the year, IRD */

I* and at least 4 characters of the last name are entered */ do

\{

/* Prompt for the Tax Year

disp_string(general, ROWTAX, 10, "Tax Year

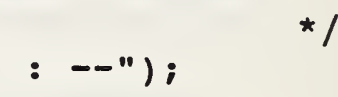

I* Prompt for the IRD

disp_string(general, ROWIRD, 10, "IRD Generated by DEQY : --");

/* Prompt for the Last Name

disp_string(general, ROWLAST, 10, "Last Name

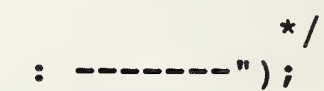

/* Prompt for the initials

disp_string(general, ROWINI, 45, " Initials: - ");

/* Prompt for the Social Security Number

disp_stringlgeneral, RowSSN, 10, "Social Security Number

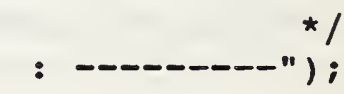

/* Prompt for the Spouse's Social security Number

disp_string(general, ROWSP, 10, "Spouse's SSN

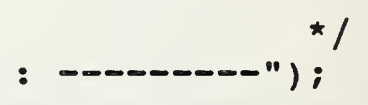

/* Prompt for the Self Employed Income

disp_string(general, ROWSEI, 10, "Self Employed Income

/* Add user direction prompts

disp_string(general, 22, 18, "ESC=EXIT ENTER=SEARCH PGUP=LAST QUERY");

/* Move to the first prompt and refreshes the screen

*/

wmove(general, ROWTAX, MINCOL);

wrefresh (general);

/* Obtain and interpret the User input until ESC (exit program)

/* or ENTER (search on query) is given

*/

input $=$ wgetch (general);

while ( (input $t=$ ESC) \& (input $!=$ ENTER) )

\{

interp(input, usrquery);

input $=\operatorname{wgetch}($ general $)$;

\}

/* check to see if the user entered enough information. If so,

$/$ * the user is done entering the query. If not, display an error

/* message and loop until the user enters the correct information

if ( (strlen(usrquery[0].year) < 2) || (strlen(usrquery[0].ird) < 2)

*

$($ strlen(usrquery[0]. lastname) $<4$ ), \&\& (input $!=$ ESC)

\{

lastlen $=$ strlen(usrquery $[0]$. lastname);

if (usrquery [0]. lastname [lastlen-1] $l={ }^{\prime},{ }^{\prime}$ )

\{

disp_erT("ERROR! MUST ENTER TAX YEAR, IRD, AND 4 LETTERS OF LAST NAME!");

clrwin (GENERALWIN);

done $=$ FALSE; 


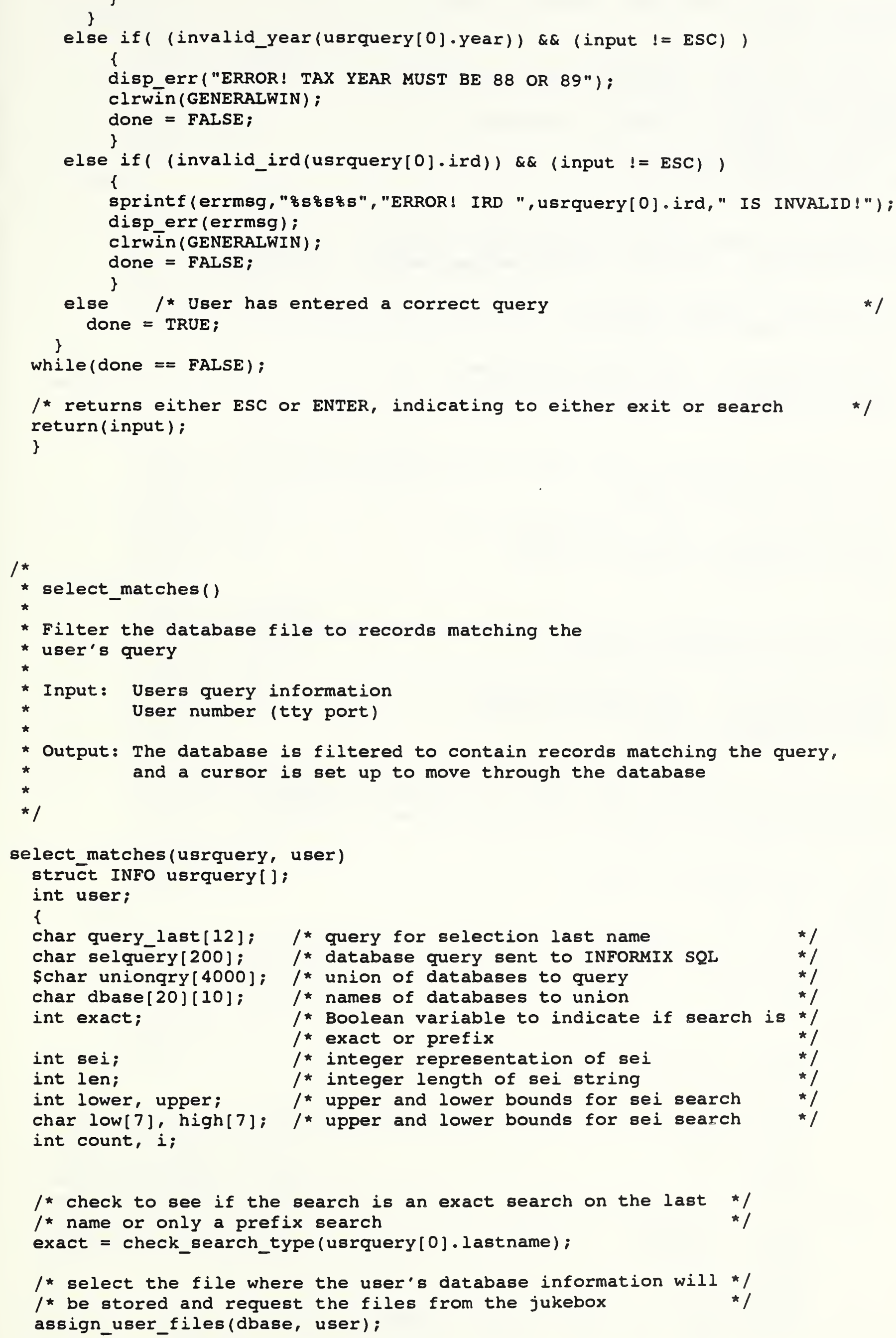




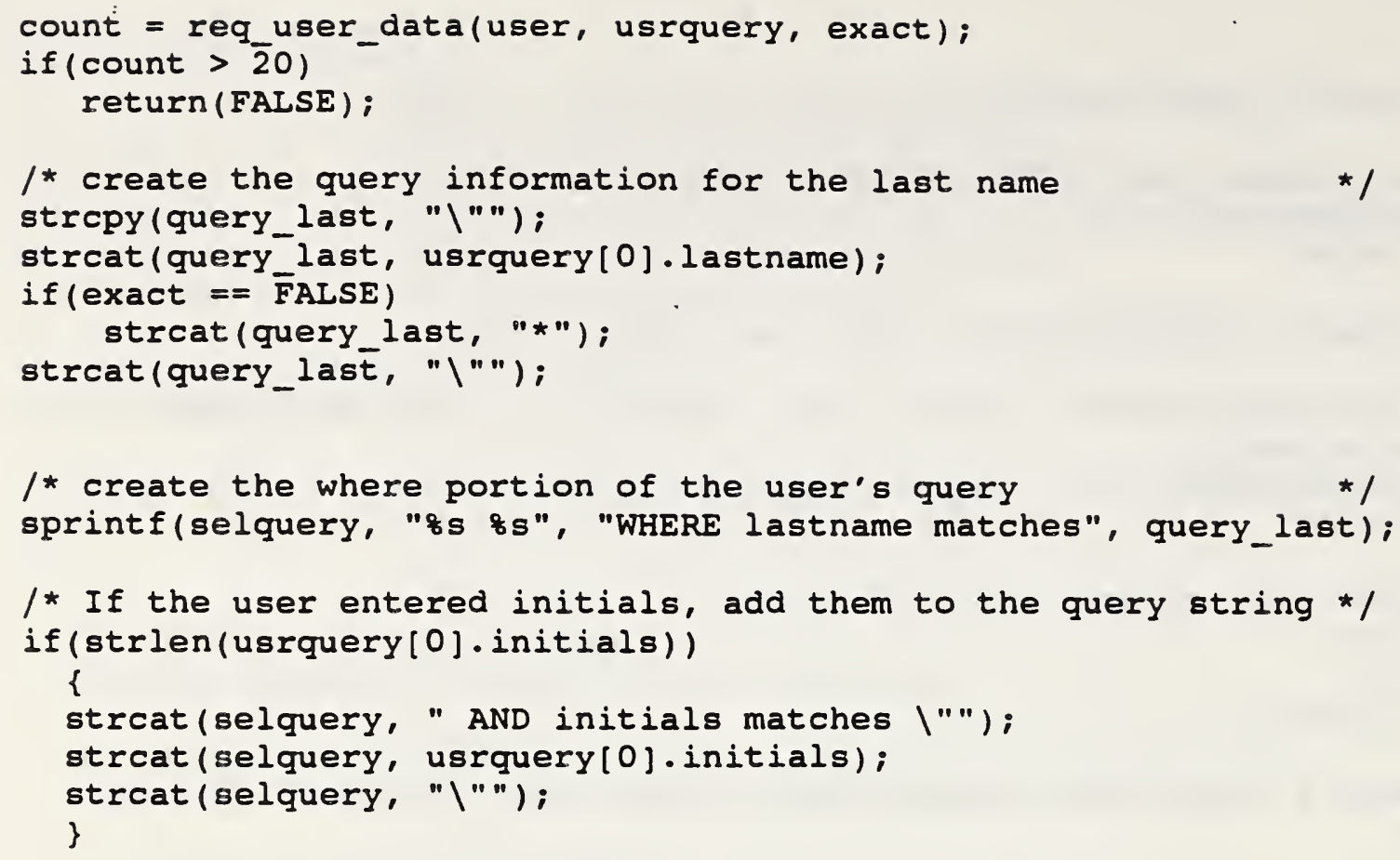




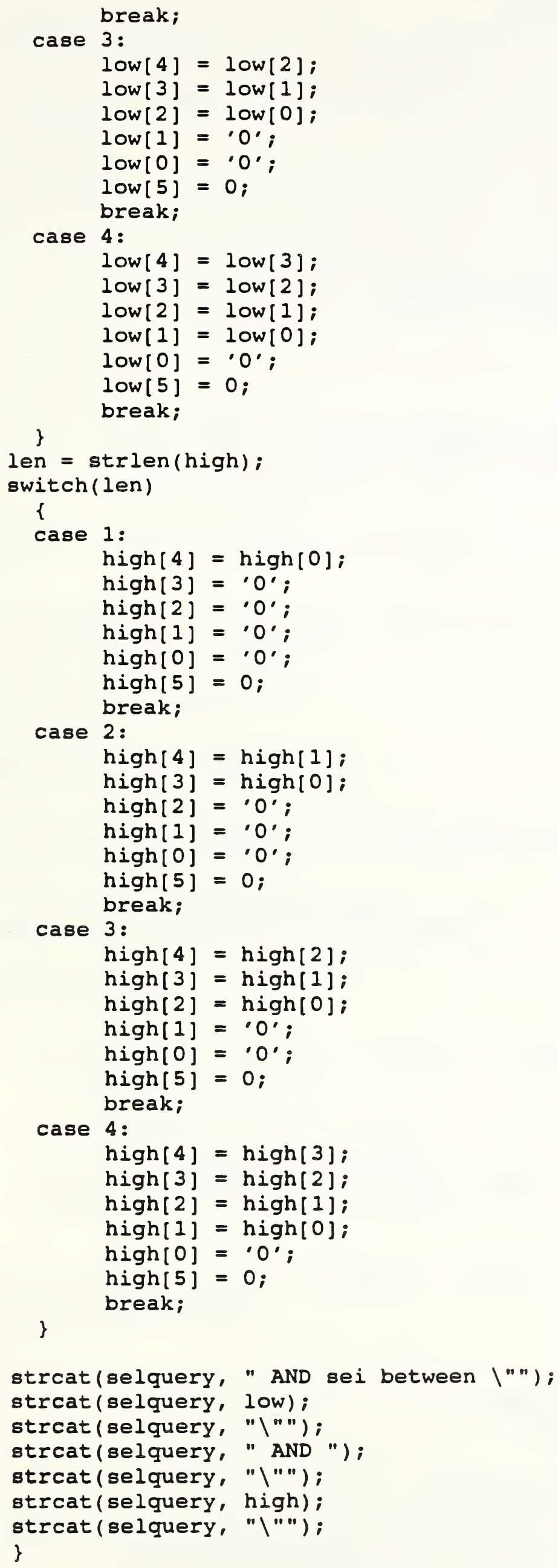

sprintf(unionqry, "is is 8 s", "SELECT * FROM", dbase[0], selquery); 


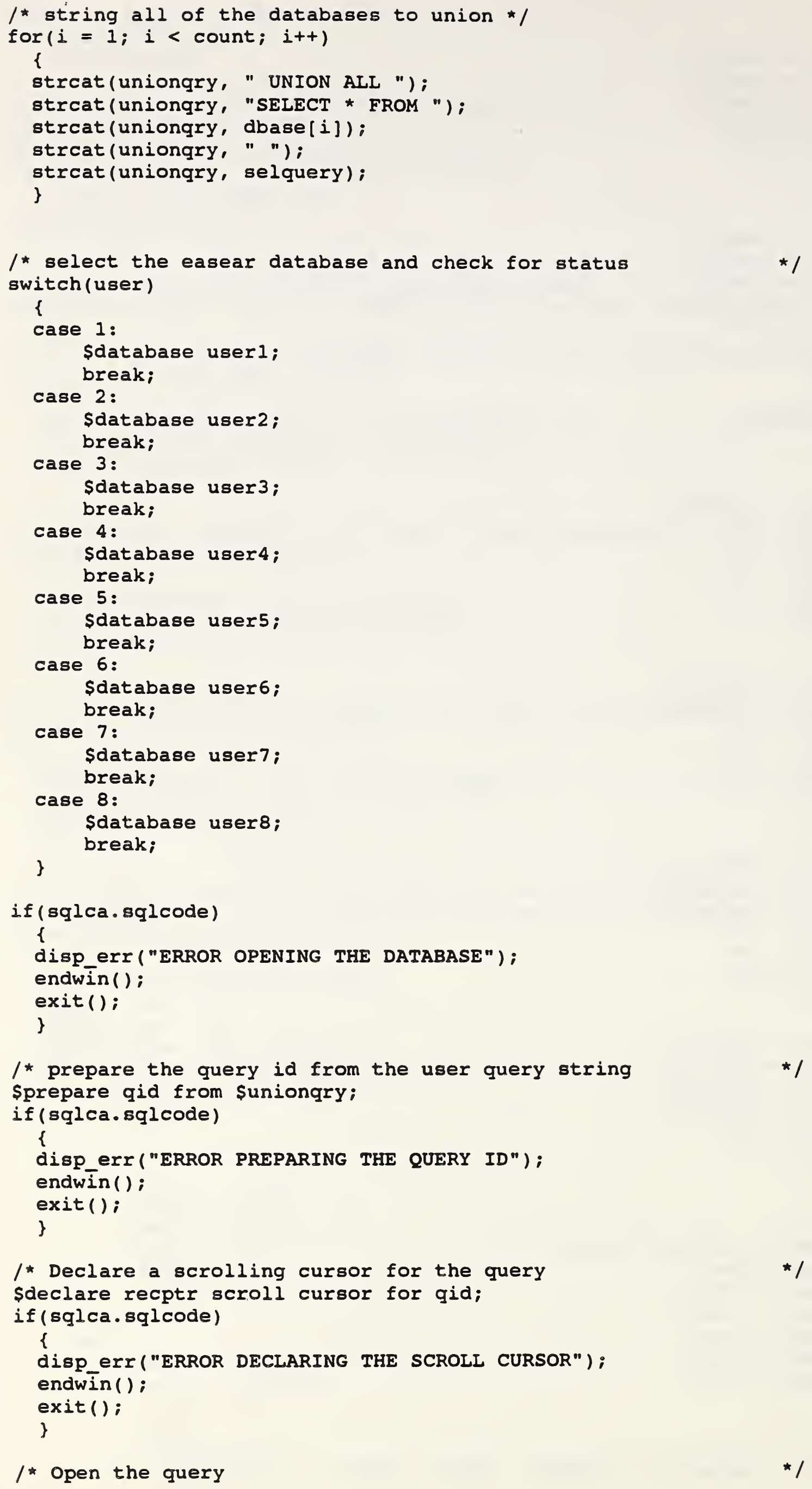




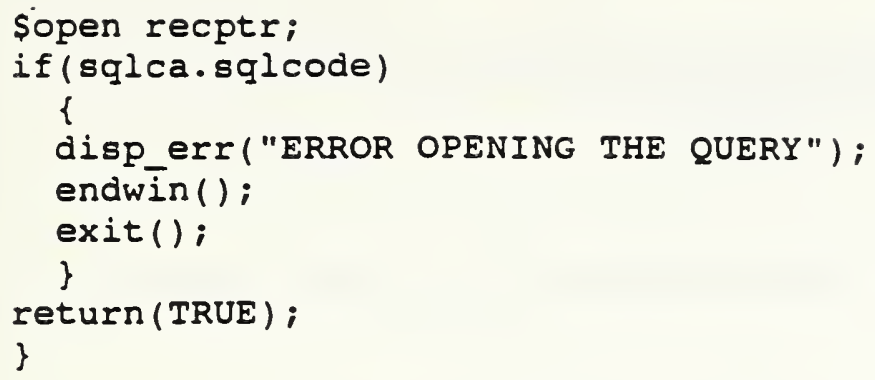

$/$ * Display Matches

* Input: structure containing the user's query information

* arrays containing the query matches loaded in memory

* current match number

* Output: the matches are displayed on the user's screen

* 1

int display matches(usrquery, querymatches, full_record, curmatch) struct QUERY querymatches [];

struct REC full_record[];

struct INFO usrquery [];

int *curmatch;

\{

int nummatches, diff;

sint topscr;

/* display the header information and the first screen of matches */ view_header(usrquery);

/* load one screen of matches

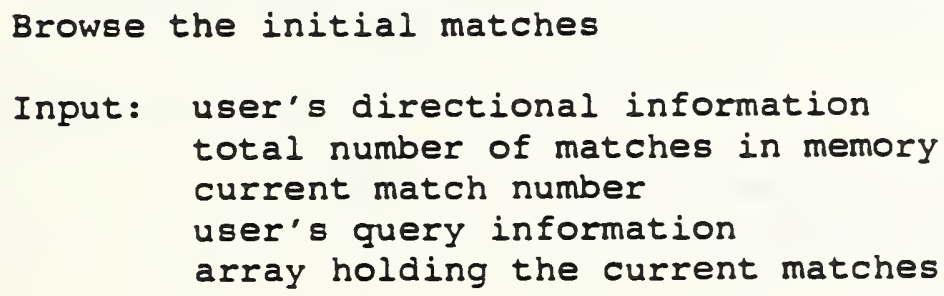


browse(input, nummatch, curmatch, usrquery, querymatches, full_record, pminfo) int * curmatch, * nummatch;

char input;

struct MANAGEINFO *pminfo;

struct QUERY querymatches [] ;

struct INFO usrquery[];

struct REC full_record[];

\{

int row, col;

int $i$;

/* get current screen cursor position

getyx(general, row, col);

/* take action based upon user's command */

switch(input)

\{

case UPARROW:

if ( IOW > FIRSTROW)

\{

* curmatch $=$ * curmatch -1 ;

wmove (general, row-2, col);

\}

break;

case DOWNARROW:

if ( (rOW < LASTROW) \&\& (*curmatch < (*nummatch-1)) ) \{

* curmatch $=$ *curmatch +1 ;

wmove (general, row +2, col) ); \}

break;

case LEFTARROW:

if ( $\mathrm{COl}>$ MINVIEW)

wmove(general, row, col-1);

break;

case RIGHTARROW:

if ( $C O I$ < MAXVIEW)

wmove (general, row, colt1);

else if ( $\operatorname{col}==$ MAXVIEW) break;

move_line (*curmatch, querymatches);

Case END: /* user requests off the screen information */ move line (*curmatch, querymatches);

break̄;

case HOME: /* restore screen to default position restore_line(*curmatch, querymatches);

break;

case ENTER: /* user requests to see detailed info on a record */ if (*nummatch $!=0$ )

\{

inc_detail_count (usrquery $[0]$-year, pminfo):

disp̄_rec(* curmatch, querymatches, full_record, pminfo);

$/$ * once the user is done, restore screen to browse mode * / restore_scr (*nummatch, *curmatch, usrquery, querymatches, full_record); \}

break;

case PAGEUP:

*nummatch $=$ view_prev_matches (querymatches, full_record,

break; cuŕmatch, *nummatch); 
case PAGEDOWN:

${ }^{*}$ nummatch $=$ view_next_matches(querymatches, full_record,

break;

\}

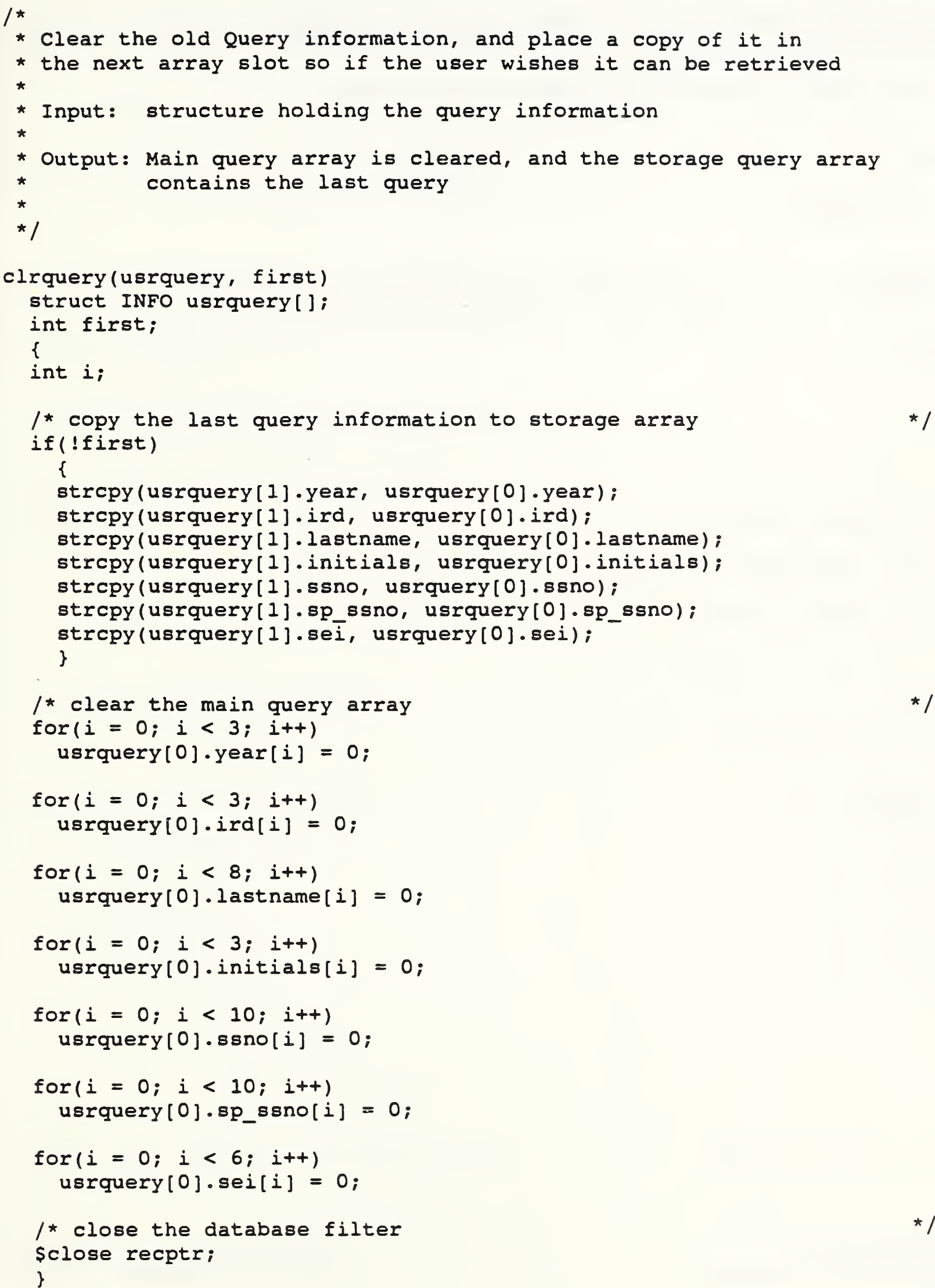




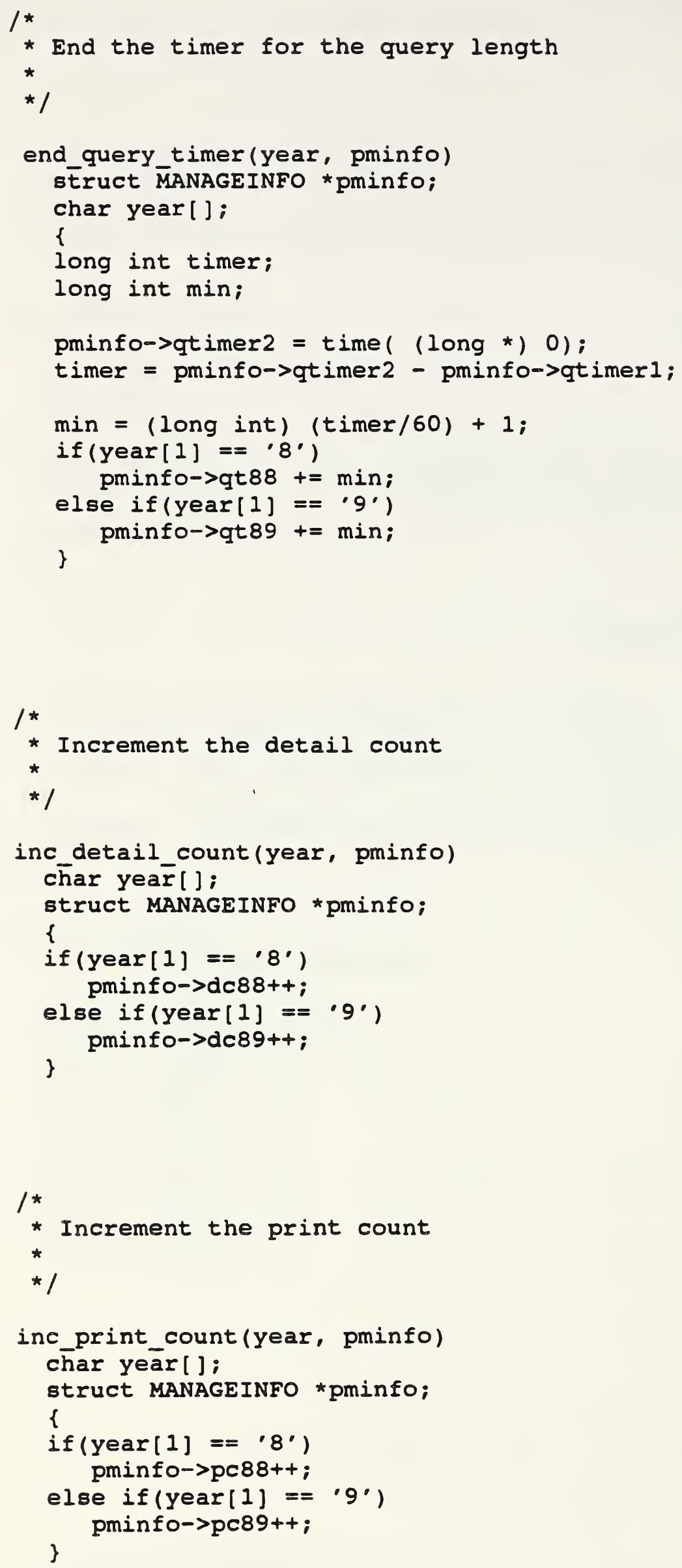


/* Open the file destroying old data if any, and write new data */ $m i=$ fopen (filename, "w");

fprintf(mi, "\&d\n", pminfo->logtime);

fprintf (mi, "8d $\backslash \mathrm{n}$ ", pminfo->logcount);

fprintf (mi, "8d $\backslash n "$, pminfo->qt88);

fprintf (mi, "zd $\backslash n "$, pminfo->qt89);

fprintf (mi, "zd $\backslash n$ ", pminfo->qc88);

fprintf (mi, "zd $\backslash n "$, pminfo->qc89);

fprintf (mi, "\&d $\backslash \mathrm{n} "$, pminfo->dc88);

fprintf(mi, "zd\n", pminfo->dc89);

fprintf(mi, "zd\n", pminfo->pc88);

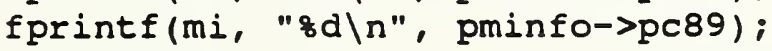

fclose $(\mathrm{mi})$;

\} 

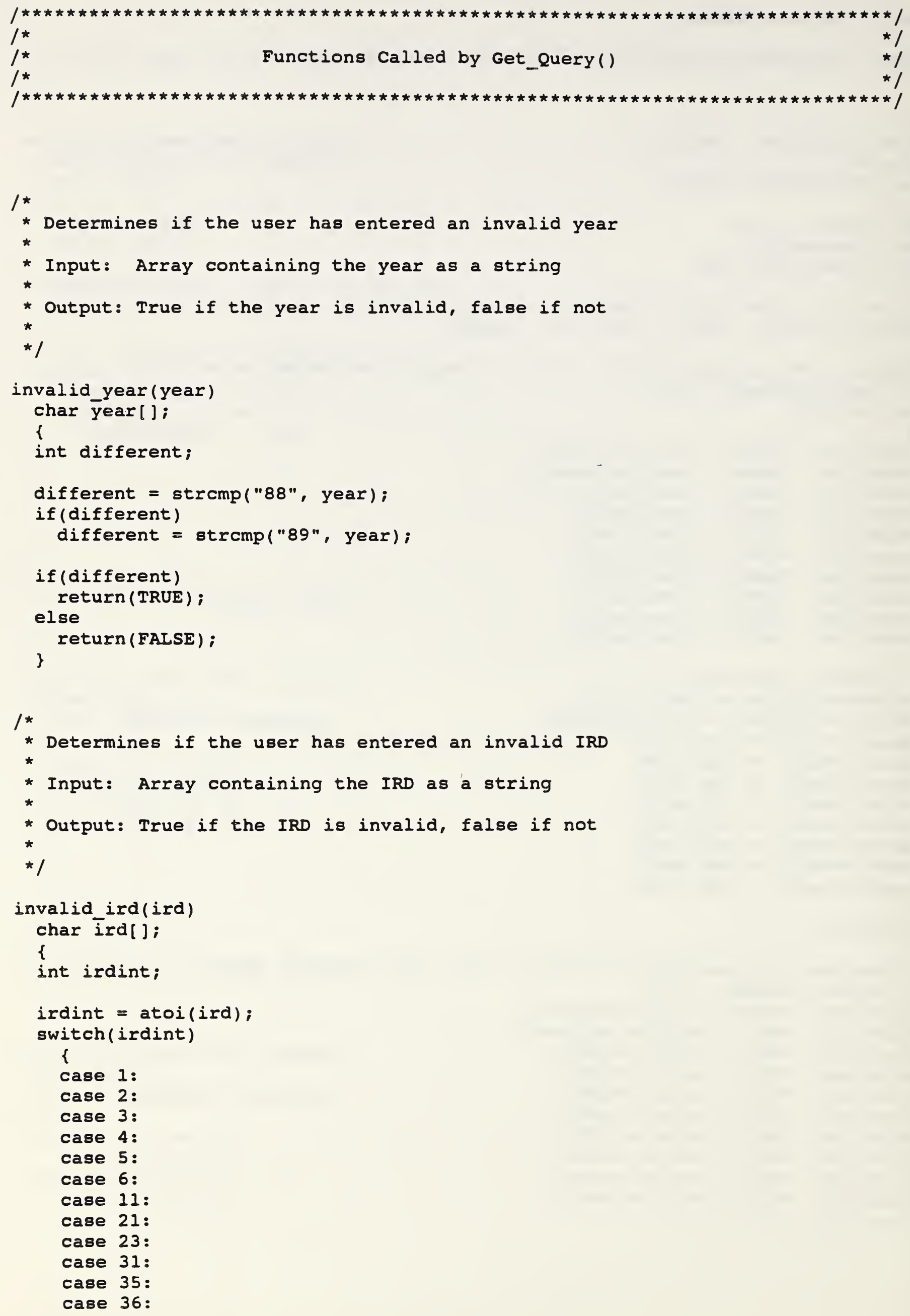


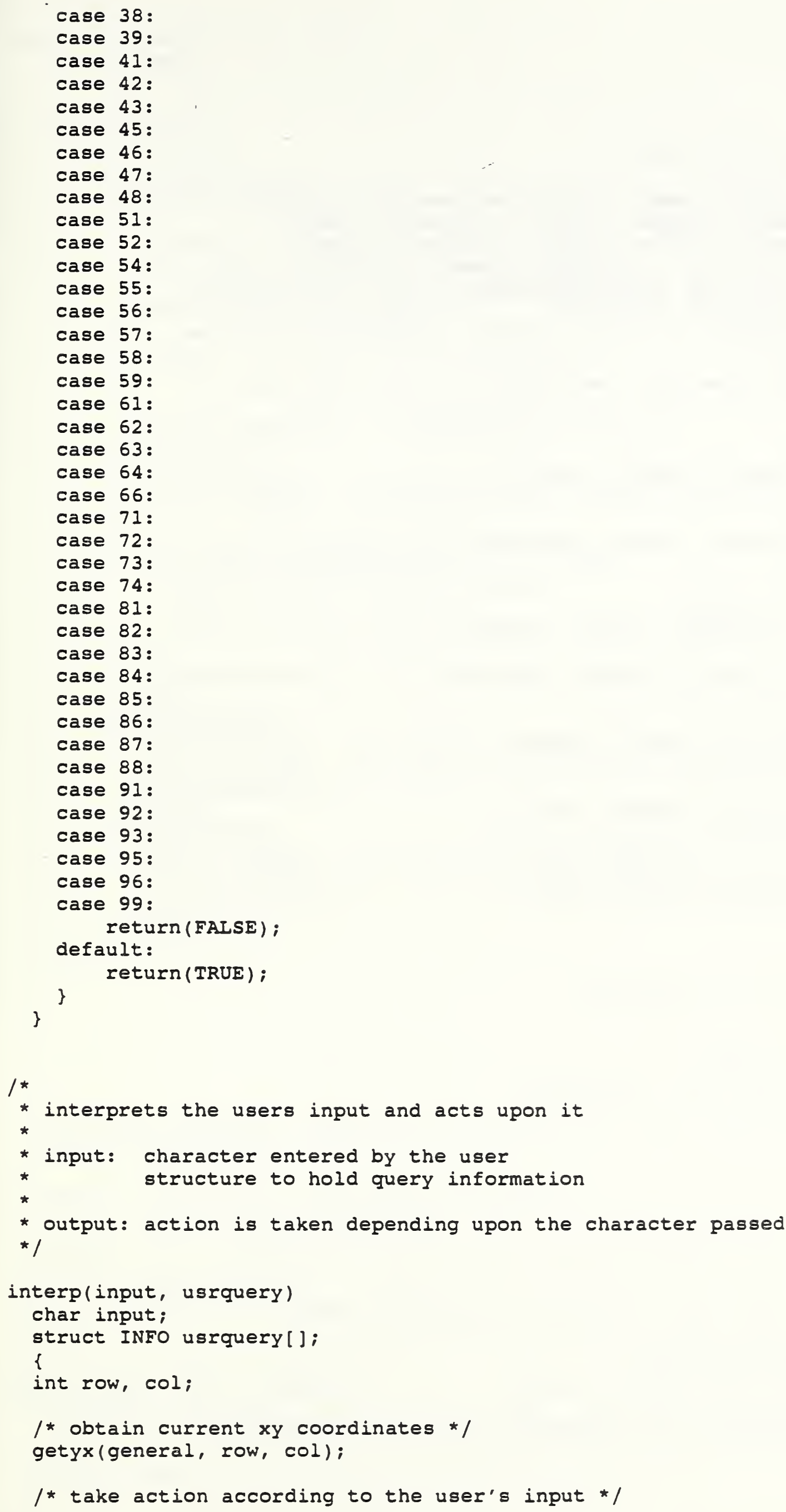


Case ROWTAX:

if $((C O)>M I N C O L) \& \&((C O L-M I N C O L)<3)) \quad / *$ In entry area? */ \{

/* delete character from screen */

wmove (general, row, col-1);

waddstr(general, "-");

wmove (general, row, col-1);

wrefresh (general);

usrquery $[0] \cdot y e a r[(\operatorname{col}-1)-M I N C O L]=0 ; \quad /$ delete character * $/$ \}

break:

case ROWIRD:

if $((C O L>M I N C O L) \& \&((C O L-M I N C O L)<3)) \quad /$ In entry area? */ \{

I* delete character from screen */

wmove (general, row, col-1);

waddstr(general, "-");

wmove(general, row, col-1);

wrefresh (general);

usrquery $[0]$.ird $[(\operatorname{COl}-1)-M I N C O L]=0 ; \quad /$ * delete character *

break;

case ROWLAST:

if $(\operatorname{Col}<\operatorname{COLINI)} \quad /$ * In last name area? */

if $(((C O I-M I N C O L)<8)$ \&\& $(C O I>M I N C O L)) \quad / *$ In entry area? *

\{

/* delete character from screen */

wmove (general, row, col-1);

waddstr(general, "-");

wmove (general, row, col-1);

wrefresh (general);

usrquery $[0]$. lastname $[(\operatorname{Col}-1)-\mathrm{MINCOL}]=0 ; /$ * Delete Char * \}

else

/ In initials area */

if $(((\operatorname{col}-\operatorname{COLINI})<3) \& \&(\operatorname{col}>\operatorname{col} I N I)) / *$ In entry area? */

\{

/* delete character from screen */

wmove (general, row, col-1);

waddstr (general, "-");

wmove (general, row, col-1);

wrefresh (general);

usrquery $[0]$.initials $[(\operatorname{col}-1)-\operatorname{col} I N I]=0 ; / *$ Delete char $* /$ \}

break;

case ROWSSN:

if $(((\mathrm{COl}-\mathrm{MINCOL})<10) \& \&(\mathrm{COI}>\mathrm{MINCOL})) /$ * In entry area? *

\{

/* delete character from screen */

wmove (general, row, col-1);

waddstr (general, "-");

wmove (general, row, col-1);

wrefresh (general);

usrquery $[0] \cdot$ ssno $[(\operatorname{COl}-1)-M I N C O L]=0 ; \quad /$ * Delete character */ \}

break;

case ROWSP:

if $(((C O I-M I N C O L)<10) \& \&(C O I>M I N C O L)) / *$ In entry area? */

\{

/* delete character from screen */

wmove (general, row, col-1):

waddstr(general, "-");

wmove (general, row, col-1);

wrefresh (general);

usrquery $[0] . s p \_s s n o[(\operatorname{col}-1)-M I N C O L]=0 ; /$ * Delete character * $/$ \}

break; 


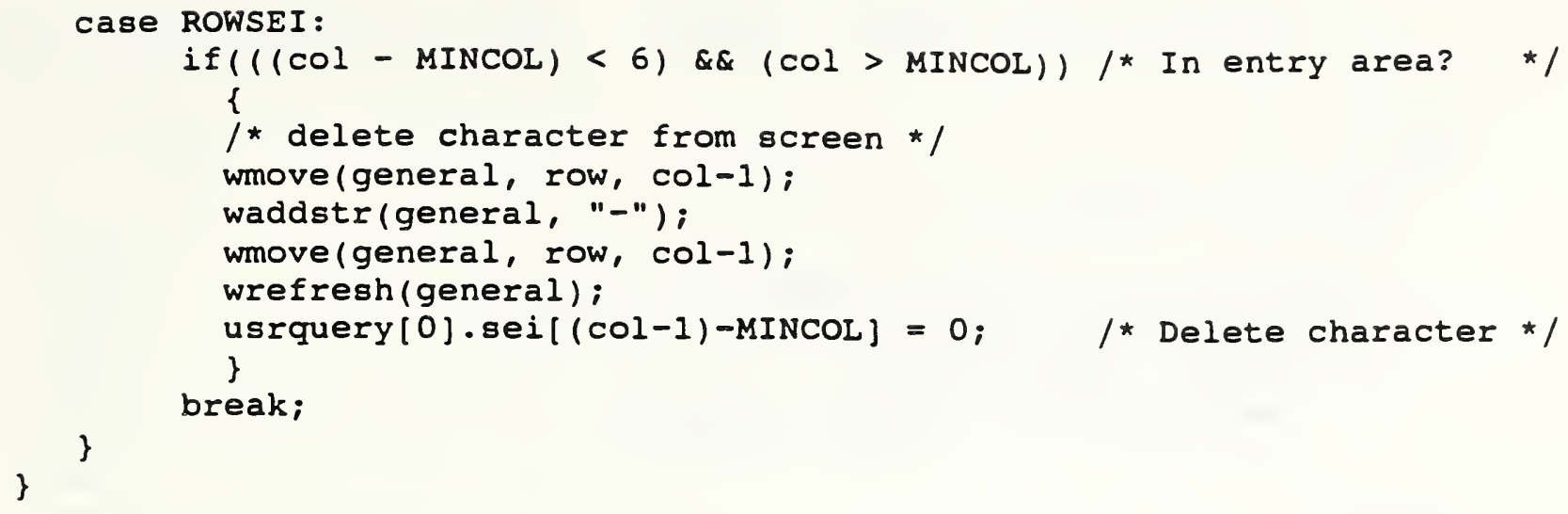




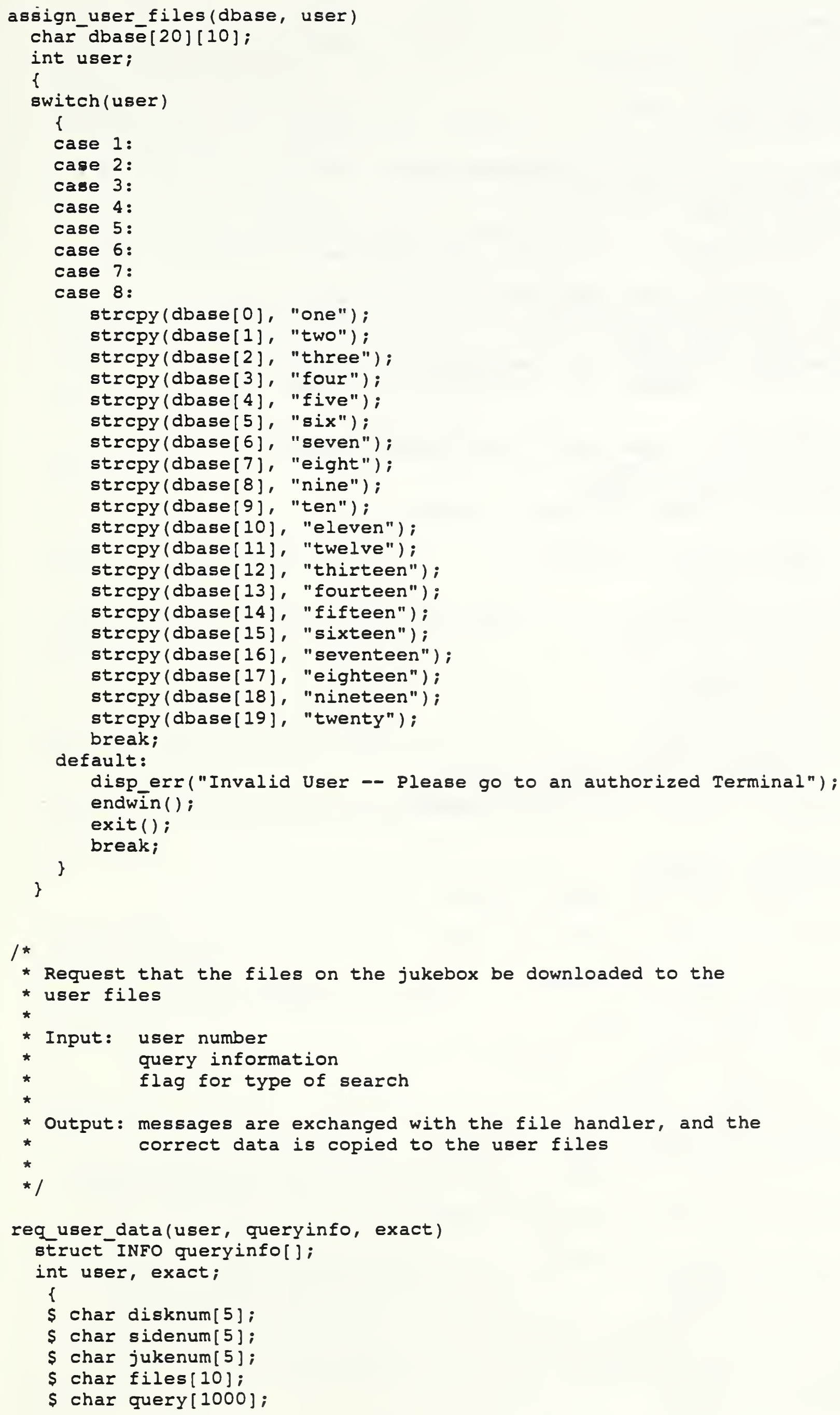




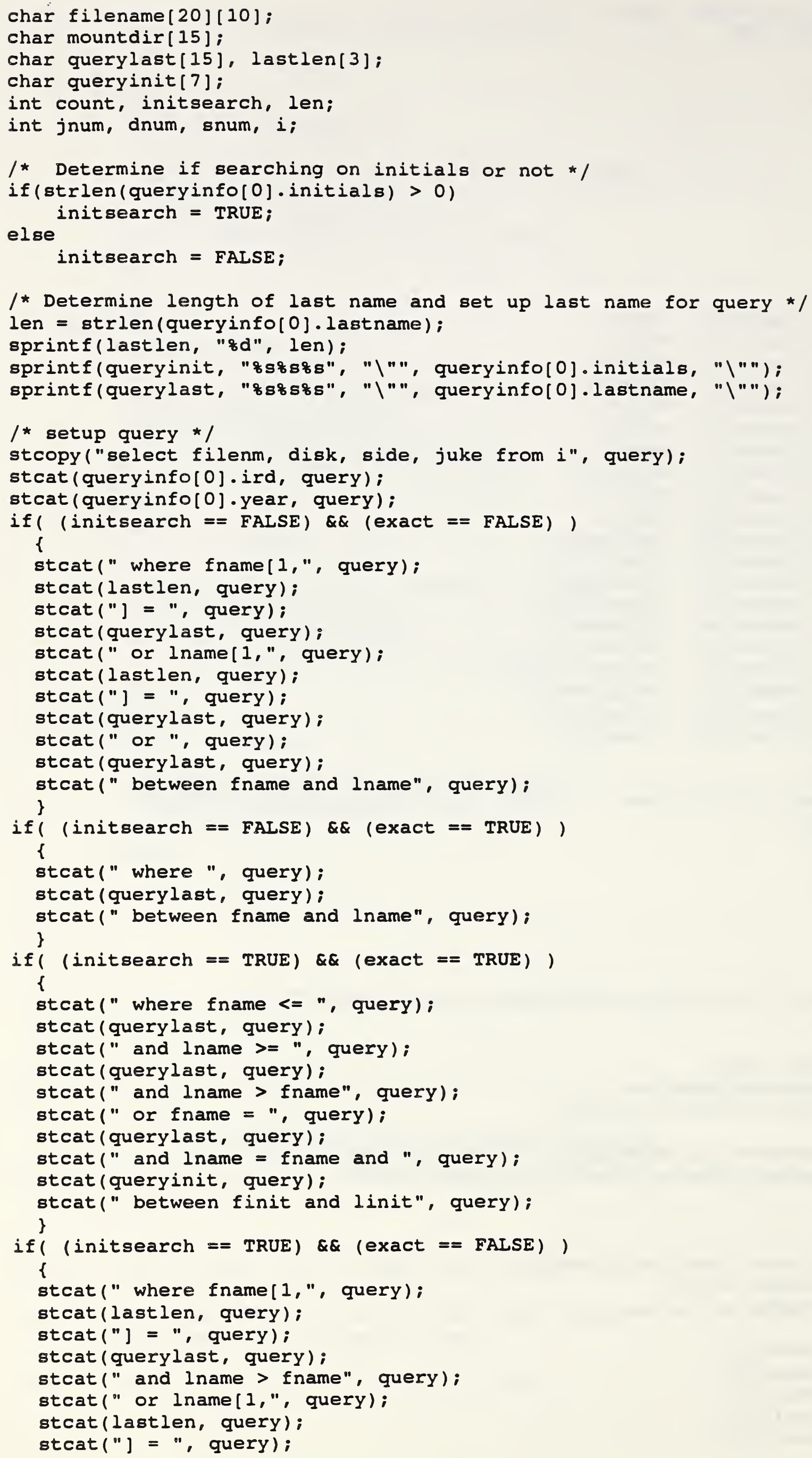




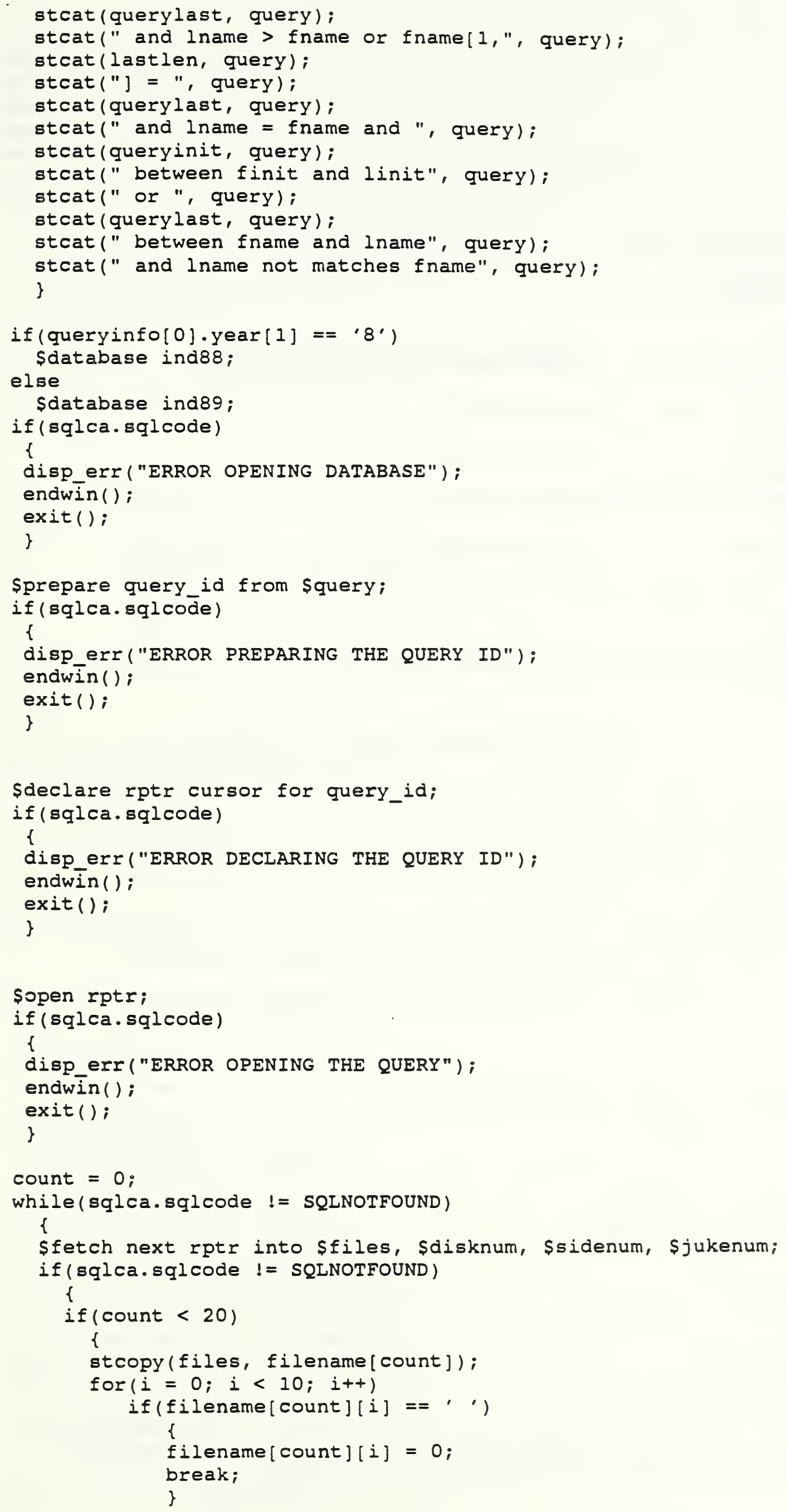




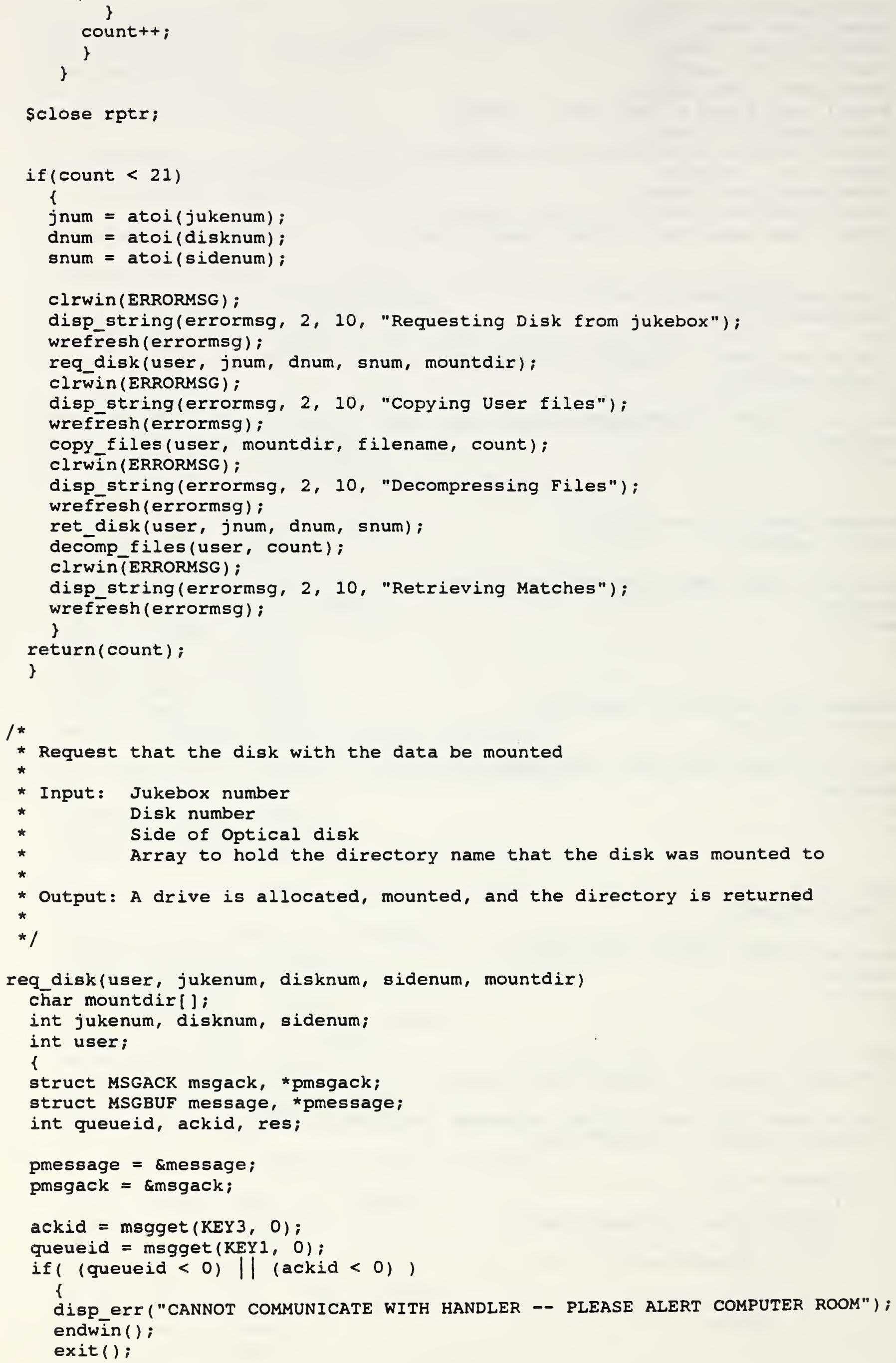




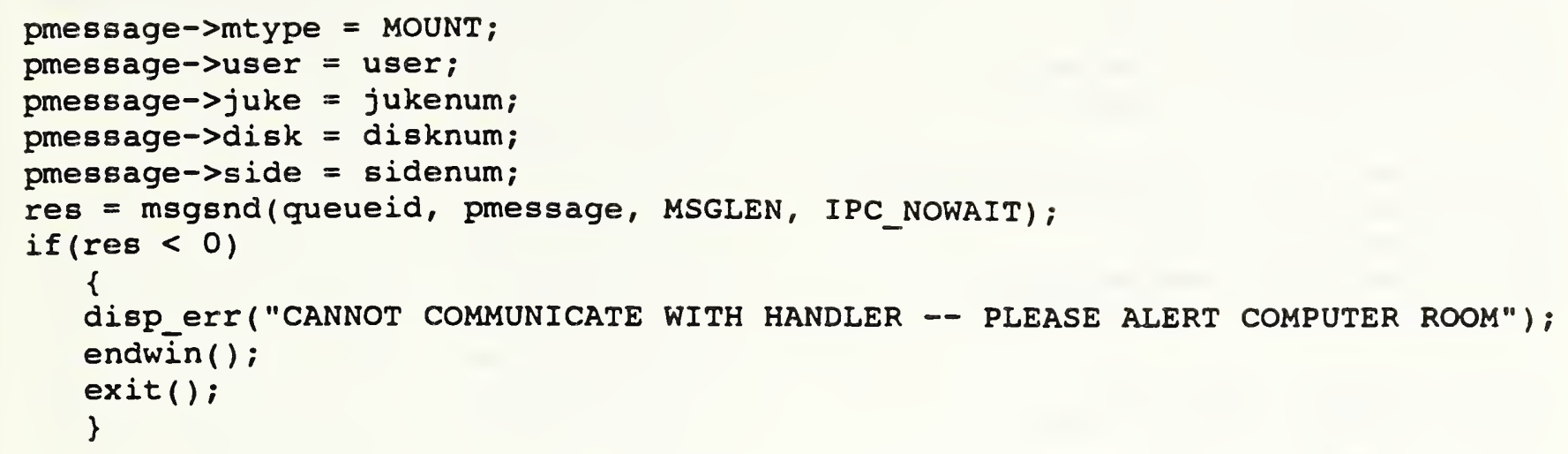

msgrcv(ackid, pmsgack, ACKLEN, ACK+user, WAIT);

strcpy (mountdir, pmsgack->mtdir);

\}

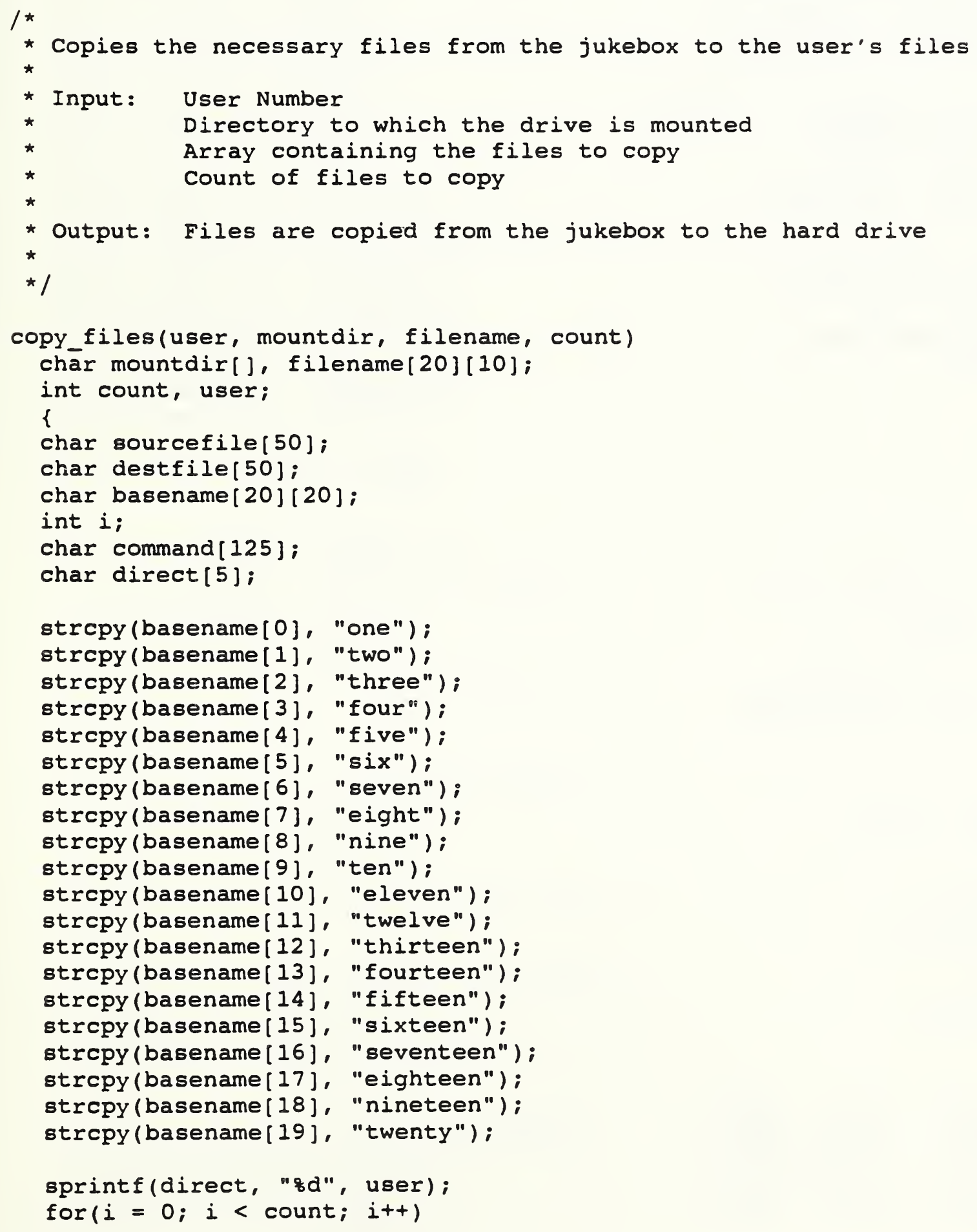



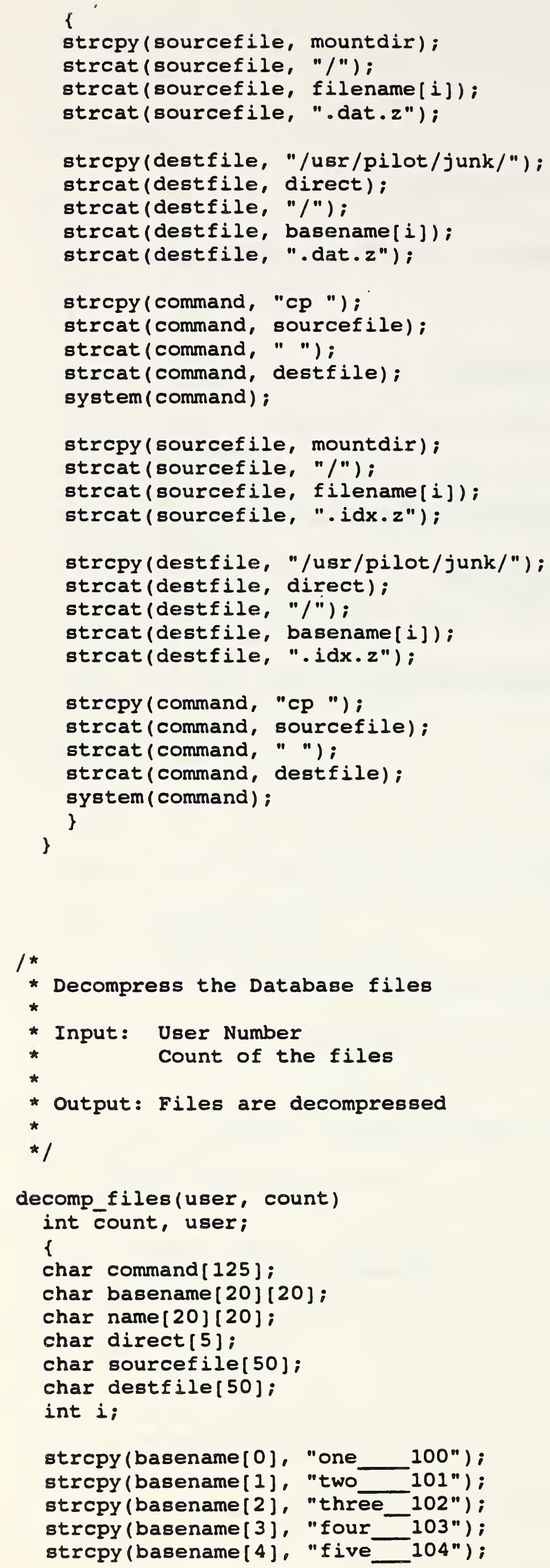


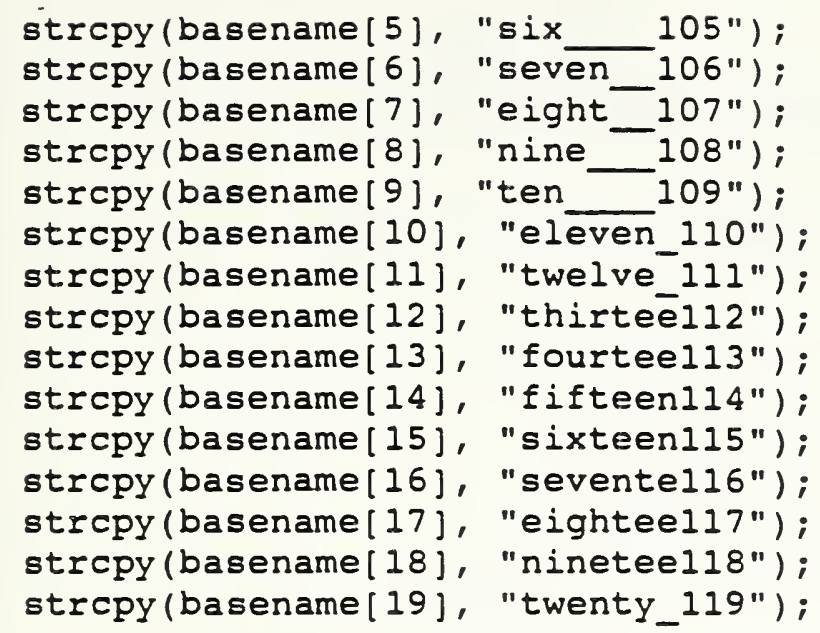

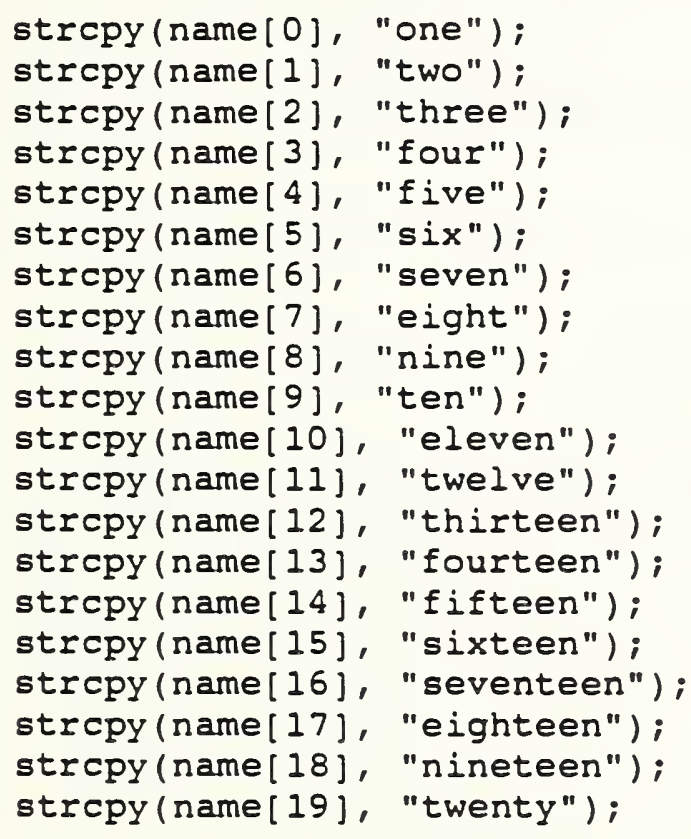


spáce_ssn(viewssn, querymatches [curmatch].sp_ssno);

disp string(general, row, 10, viewssn);

disp_string(general, row, 22, querymatches[curmatch].sp_sei);

disp_string(general, row, 28, querymatches[curmatch].fuIlname);

wrefresh (general);

\}

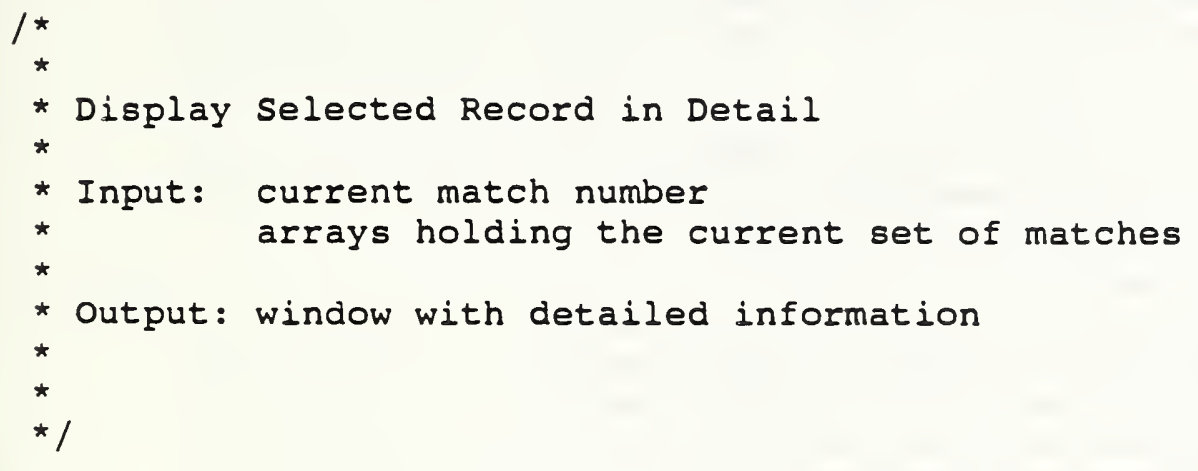

disp_rec(curmatch, querymatches, full_record, pminfo)

struct QUERY querymatches[];

struct REC full_record[];

struct MANAGEINF̄o *pminfo;

int curmatch;

\{

int $j, i$;

char input;

char viewssn[12];

/* Bring up the detail window */

show panel (pdetail);

clrwin(DETAIIWIN);

/ * Display name */

disp_string(detail, 2, 2, "Name : ");

waddstr(detail, querymatches [curmatch].fullname);

/* Display Address */

disp_string(detail, 3, 2, "Address: "):

waddstr(detail, full record[curmatch].st_add);

disp_string(detail, $\overline{4}, 12$, full_record[cürmatch].city_st);

waddstr(detail, " ");

waddstr(detail, full_record[curmatch].zip);

/ * Display Column Headings * /

disp_string(detail, 6, 4, "Name");

disp_string(detail, 6, 14, "SSN");

disp_string(detail, 6, 27, "NESEB");

disp_string(detail, 6, 33, "NESEF");

disp_string(detail, 6, 39, "WAGES");

disp_string(detail, 6, 45, "SEI");

disp_string(detail, 6, 52, "YEARQU");

disp_string(detail, 6, 60, "ST");

disp_string(detail, 6, 64, "DLN/EIN IND CD");

disp string(detail, 7, 2, "--");

for $(\bar{j}=0 ; j<37 ; j++)$

waddstr(detail, "--");

/ * Display First row */

disp string(detail, 8, 2, querymatches (curmatch].lastname);

disp_string(detail, 8, 10, querymatches[curmatch].initials);

spacéssn(viewssn, querymatches[curmatch].ssno);

disp_string(detail, 8, 14, viewssn); 
disp_string(detail, 8, 27, full_record(curmatch).neseb);

disp_string(detail, 8, 33, full_record[curmatch].nesef);

disp_string(detail, 8, 39, full_record[curmatch].wages);

disp_string(detail, 8, 45, querymatches [curmatch].sei);

disp_string (detail, 8, 52, querymatches [curmatch].year);

waddstr(detail, full_record[curmatch].month);

waddstr(detail, full_record[curmatch].option_cd);

waddstr(detail, full_record[curmatch].se_quar);

disp_string(detail, $\overline{8}, 60$, querymatches [ curmatch].ird);

disp_string(detail, 8, 64, full_record(curmatch).dln);

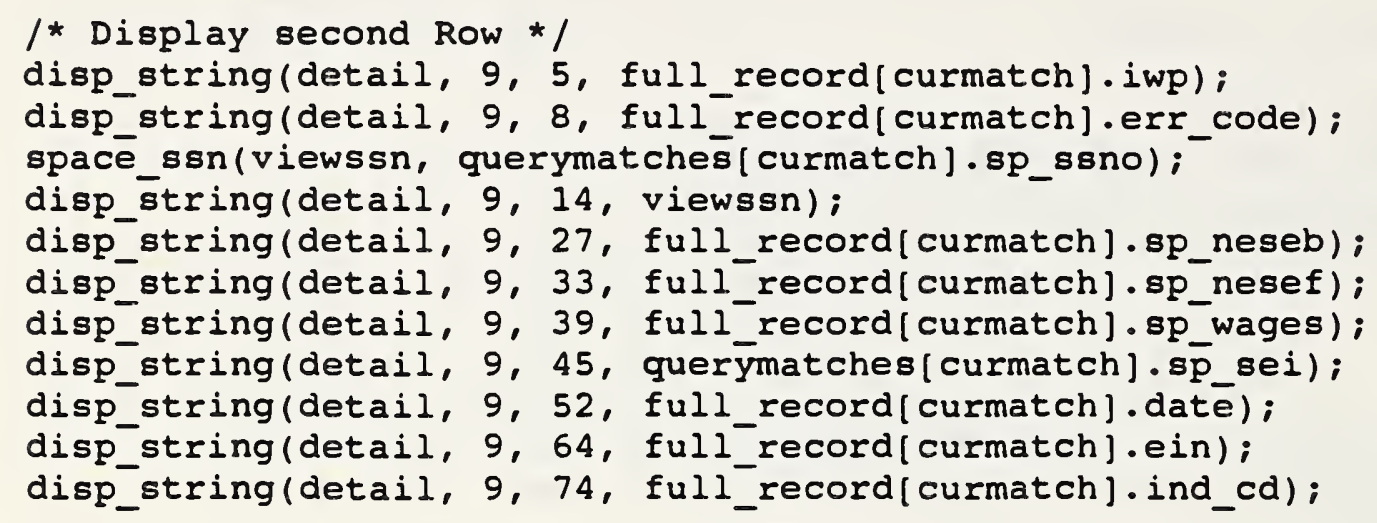


printf("Name : is $\backslash n$ ", querymatches[curmatch].fullname);

printf ("Address: is $\backslash \mathrm{n}$ ", full_record[curmatch].st_add);

printf" of os $s \backslash n \backslash n "$ ",

full_record[curmatch].city_st, full_record[curmatch].zip);

printf("Name SSN NESEB NESEF WAGES SEI YEARQU ST DLN/EIN IND CD $\backslash \mathrm{n}$ ");

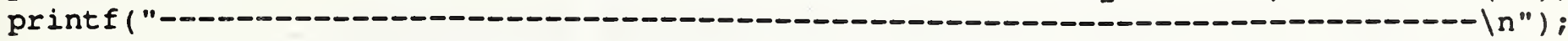

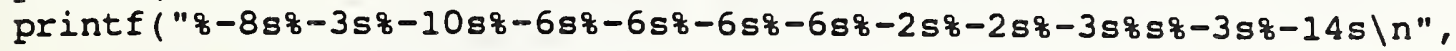
querymatches [curmatch]. lastname, querymatches [curmatch].initials, querymatches [curmatch].ssno, full_record[curmatch].neseb, full_record [curmatch]. nesef, full_record[curmatch].wages, querymatches [curmatch] • sei, querymatches [curmatch] •year, full_record[curmatch].month, full_record[curmatch].se_quar, full-record [curmatch]. option_cd, querȳmatches [curmatch].ird, full_record [curmatch].dln);

printf(" $z-3 s z-3 s z 118 z 6 s q-6 s q-6 s z-6 s z-10 s z-10 s q-5 s \backslash n \backslash f "$, full_record [curmatch]. iwp, full_record[curmatch].err_code, querȳmatches [curmatch] .sp_ssno, full_record[curmatch].sp_neseb, full_record[curmatch].sp_ñesef, full_record[curmatch].sp_wages, querȳmatches [curmatch] .sp_sei, full_record [curmatch].date, full_record[curmatch].ein, full_recörd[curmatch].ind_cd);

/* return the output to the screen system("tput mc4");

\}

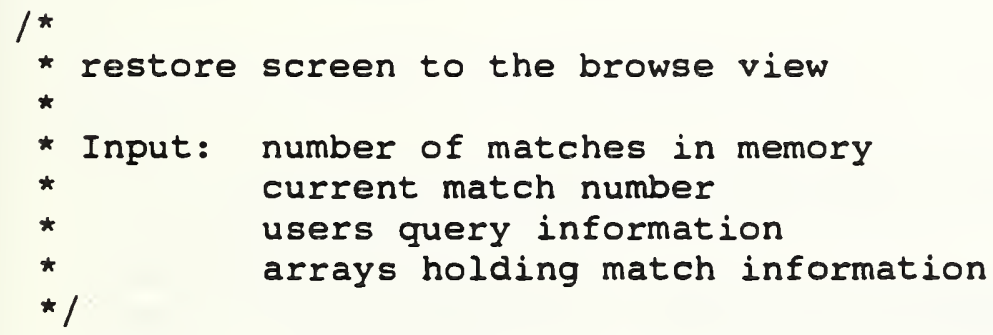

restore_scr(nummatch, curmatch, usrquery, querymatches, full_record) int nummatch, curmatch;

struct INFO usrquery[];

struct REC full_record[];

struct QUERY quérymatches[];

\{

int pgmatch, rownum;

/ * Display the header information */

view_header (usrquery);

/ * Display current page of matches */

disp_screen(querymatches, nummatch);

/* Move cursor to correct line on screen */

rownum $=($ curmatch $* 2)+6 ;$

wmove (general, rownum, 2);

\}

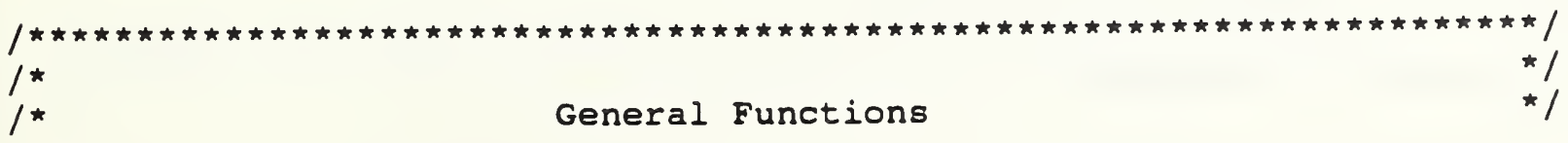




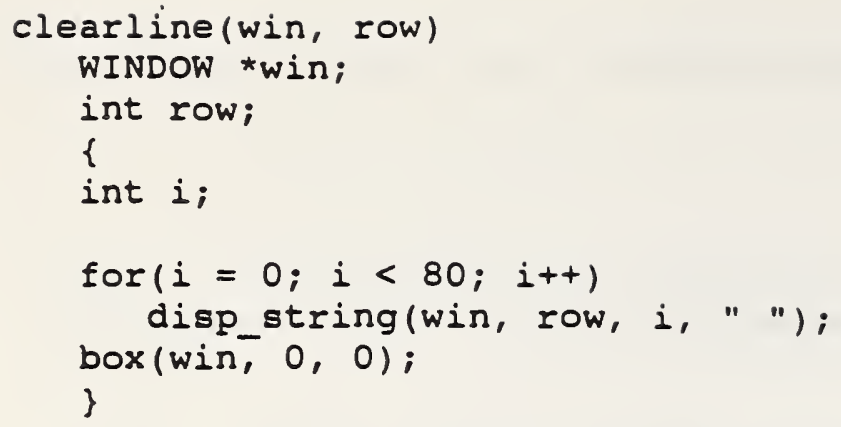

Display matches currently in memory

input: array containing browse information on matches number of matches in memory

output: a screen of matches is displayed

$\star /$

disp_screen (querymatches, num)

struct QUERY querymatches[];

int num;

\{

int i, row, col;

char buffer[21];

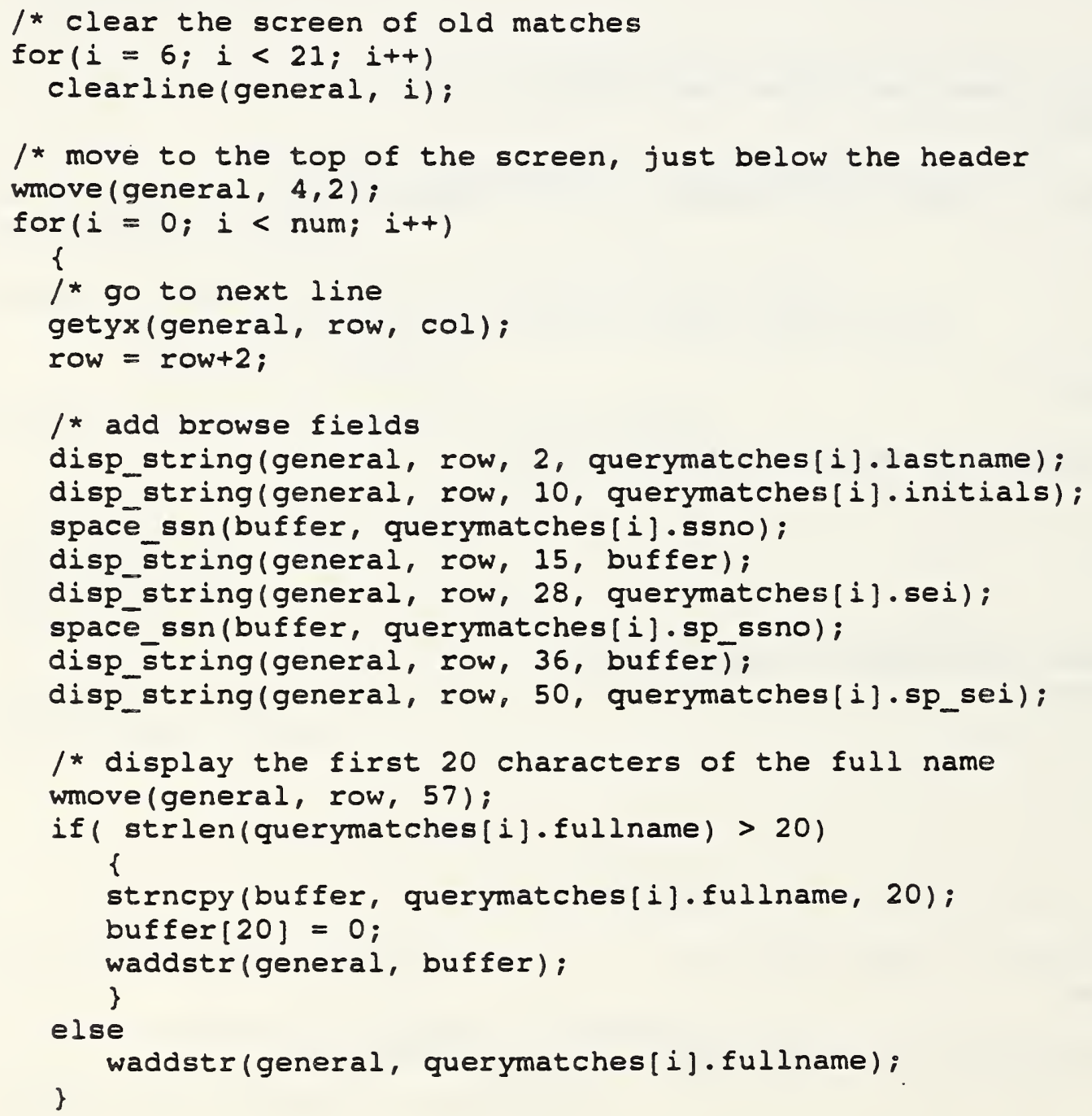


/* Add user directional prompts

/* Move to the first row and display the matches

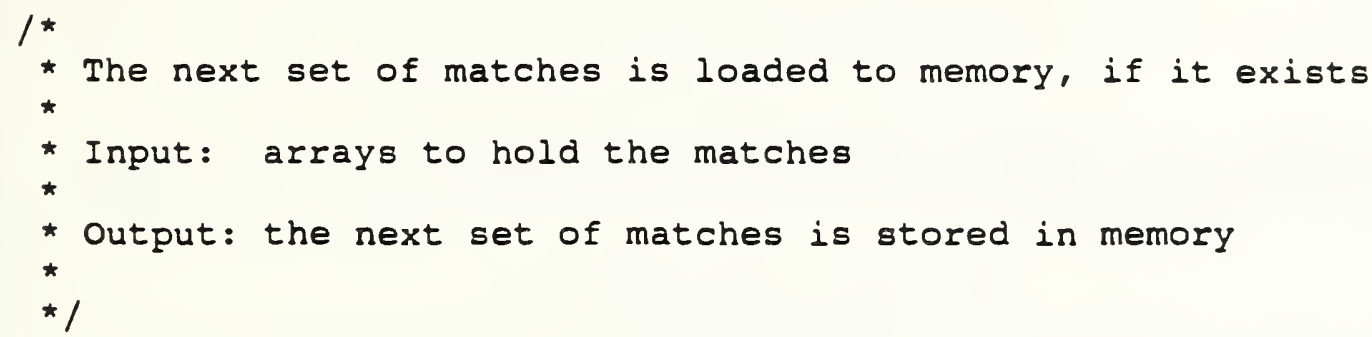

int load_matches(querymatches, full_record)

struct QUERY querymatches [];

struct REC full_record[];

\{

int $i, j$;

/* Get a match from the file

for $(i=0 ; i<\operatorname{MATCHSCR} ; i++)$

\{

\$fetch next recptr into \$retrvar.lastname, \$retrvar.initials,

\$retrvar.ssno, \$retrvar.neseb, \$retrvar.nesef, \$retrvar.wages, \$retrvar.sei, \$retrvar.year, Sretrvar.month, \$retrvar.se_quar, \$retrvar.option cd, sretrvar.ird, sretrvar.dln, \$retrvar.full name, sretrvar.iwp, sretrvar.err_code, sretrvar.sp_ssno, \$retrvar.sp̄neseb, \$retrvar.sp_nesef, \$retrvar.sp_wages, \$retrvar.sp_sei, \$retrvar.date_rcvd, \$retrvar.ein, \$retrvar.ind_cd, \$retrvar.st_add, Sretrvar.city_st, \$retrvar.zip;

/* If no matches exist, exit the loop

if (sqlca.sqlcode $==$ SQLNOTFOUND)

break;

/* Copy the match from the host variable to the array

stcopy (retrvar.lastname, querymatches[i].lastname);

stcopy(retrvar.initials, querymatches[i].initials);

stcopy (retrvar.ssno, querymatches[i].ssno);

stcopy (retrvar.neseb, full_record[i].neseb);

stcopy (retrvar.nesef, full_record[i].nesef);

stcopy (retrvar.wages, full record[i].wages);

stcopy (retrvar.sei, querymatches[i].sei);

stcopy (retrvar.year, querymatches[i].year);

stcopy (retrvar.month, full record[i].month);

stcopy (retrvar.se_quar, fuIl_record[i].se_quar);

stcopy (retrvar.option_cd, fuIl record[i].option_cd);

stcopy (retrvar.ird, querymatchés[i].ird);

stcopy (retrvar.dln, full recordil.diln);

stcopy (retrvar.full_name, querymatches[i].fullname);

stcopy (retrvar.iwp, full_record[i].iwp);

stcopy (retrvar.err_code, full_record [i].err_code);

stcopy (retrvar.sp_ssno, querymatches[i].sp_ssno);

stcopy (retrvar.sp_neseb, full_record (i).sp_neseb);

stcopy (retrvar.sp_nesef, full_record[i].sp_nesef);

stcopy (retrvar.sp_wages, full_record[i].sp_wages);

stcopy (retrvar.sp_sei, querymätches[i].sp_sei); 
stcopy (retrvar.date revd, full_record[i].date); stcopy (retrvar.ein, full recordi[i].ein); stcopy (retrvar.ind_cd, füll_record[i].ind_cd); stcopy (retrvar.st_âdd, full_record[i].st_àdd);

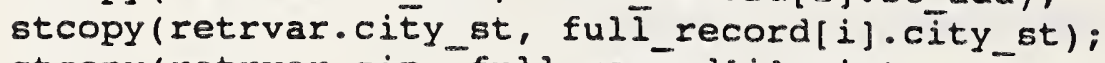
\} stcopy (retrvar.zip, full_record[i].zip);

$/$ * return the number of matches found return(i);

\}

/*

* The previous set of matches is loaded to memory, if it exists

*

* Input: arrays to hold the matches

*

* Output: the previous set of matches is stored in memory

$\star$

* /

int load_prev_matches(querymatches, full_record)

struCE QUĒRY querymatches[];

struct REC full_record[];

\{

int $i, j ;$

/* Get one match from the data file

for $(i=1 ; i<=\operatorname{MATCHSCR} ; i++)$

\{

\$fetch previous recptr into \$retrvar.lastname, \$retrvar.initials,

\$retrvar.ssno, \$retrvar.neseb, \$retrvar.nesef, \$retrvar.wages,

\$retrvar.sei, \$retrvar.year, \$retrvar.month, \$retrvar.se_quar, \$retrvar.option_cd, \$retrvar.ird, \$retrvar.dln, \$retrvar.full name, \$retrvar.iwp, \$retrvar.err_code, \$retrvar.sp_ssno, \$retrvar.sp̄neseb, \$retrvar.sp_nesef, \$retrvar̄.sp_wages, \$retrvār.sp_sei, sretrvar.date_rcvd, \$retrvar.ein, \$retrvar.ind_cd, sretrvar.st_add, \$retrvar.city_st, \$retrvar.zip;

/* If no matches exits, exit the loop

if (sqlca.sqlcode $==$ SQLNOTFOUND)

break;

I* Copy the match from the host variable to the arrays

stcopy (retrvar. lastname, querymatches[MATCHSCR - i].lastname); stcopy (retrvar.initials, querymatches [MATCHSCR - i].initials); stcopy (retrvar.ssno, querymatches [MATCHSCR - i].ssno); stcopy (retrvar.neseb, full record [MATCHSCR - i].neseb); stcopy (retrvar.nesef, full-record[MATCHSCR - i].nesef); stcopy (retrvar.wages, full-record[MATCHSCR - i].wages); stcopy (retrvar.sei, querymätches [MATCHSCR - i].sei); stcopy (retrvar.year, querymatches [MATCHSCR - i] -year); stcopy (retrvar.month, full record [MATCHSCR - i.].month); stcopy (retrvar.se_quar, fuIl record [MATCHSCR - i].se_quar); stcopy (retrvar.option_cd, fuIl_record[MATCHSCR - i].option_cd); stcopy (retrvar.ird, quererymatchēs [MATCHSCR - i].ird); stcopy (retrvar.dln, full_record[MATCHSCR - i].dln); stcopy (retrvar.full name, querymatches [MATCHSCR - i].fullname); stcopy (retrvar.iwp, full_record[MATCHSCR - i].iwp); stcopy (retrvar.err_code, full_record[MATCHSCR - i] .err_code); stcopy (retrvar.sp_ssno, querymatches [MATCHSCR - i].sp_ssno); stcopy (retrvar.sp_neseb, full_record[MATCHSCR - i].sp_neseb); stcopy (retrvar.sp_nesef, full_record[MATCHSCR - i].sp_nesef); 
stcopy (retrvar.sp_wages, full_record[MATCHSCR - i].sp_wages);

stcopy (retrvar.sp_sei, querymatches[MATCHSCR - i].sp_sei);

stcopy (retrvar.date_rcvd, full_record[MATCHSCR - i]. date);

stcopy (retrvar.ein, full_record̄[MATCHSCR - i].ein);

stcopy (retrvar.ind_cd, full_record[MATCHSCR - i].ind cd);

stcopy (retrvar.st_add, full_record[MATCHSCR - i].st_àdd);

stcopy (retrvar.city_st, fuli_record[MATCHSCR - i].city_st);

\}

stcopy (retrvar.zip, full_recōrd[MATCHSCR - i].zip);

return(i-1);

\}

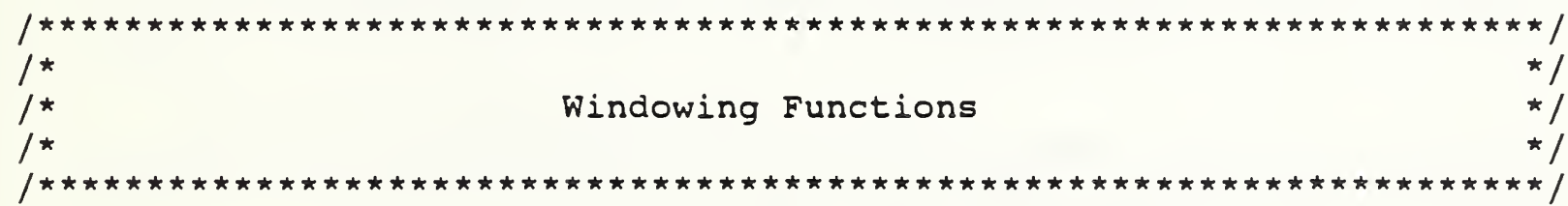

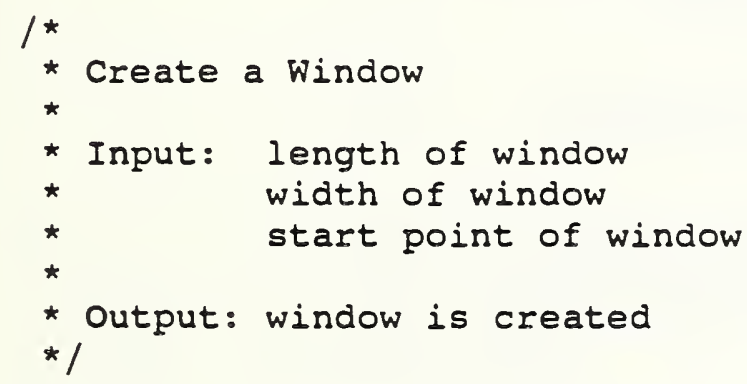

WINDOW *create(length, width, y_start, $x_{\text {_start) }}$

int length, width, y_start, $x_{-}$start;

\{

WINDOW *win;

/* create the window * /

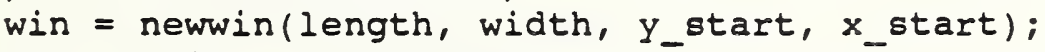

return(win);

\}

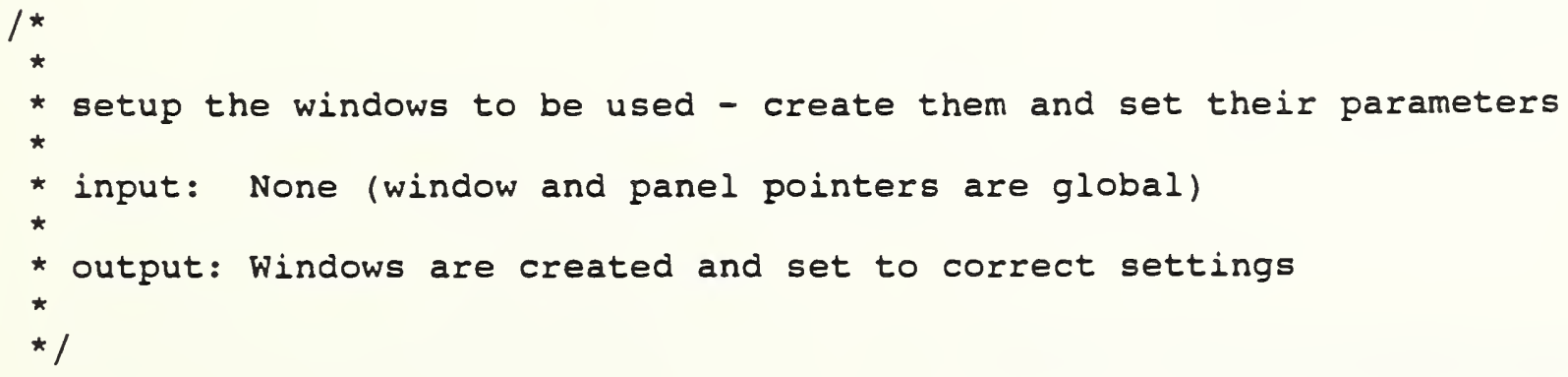

setup_windows ( )

\{

WINDOW *Create();

/* Create the background, detail, and error message windows */

detail = $\operatorname{create}(16,80,5,0)$;

general $=\operatorname{create}(25,80,0,0)$;

errormsg $=\operatorname{create}(5,80,7,0)$; 


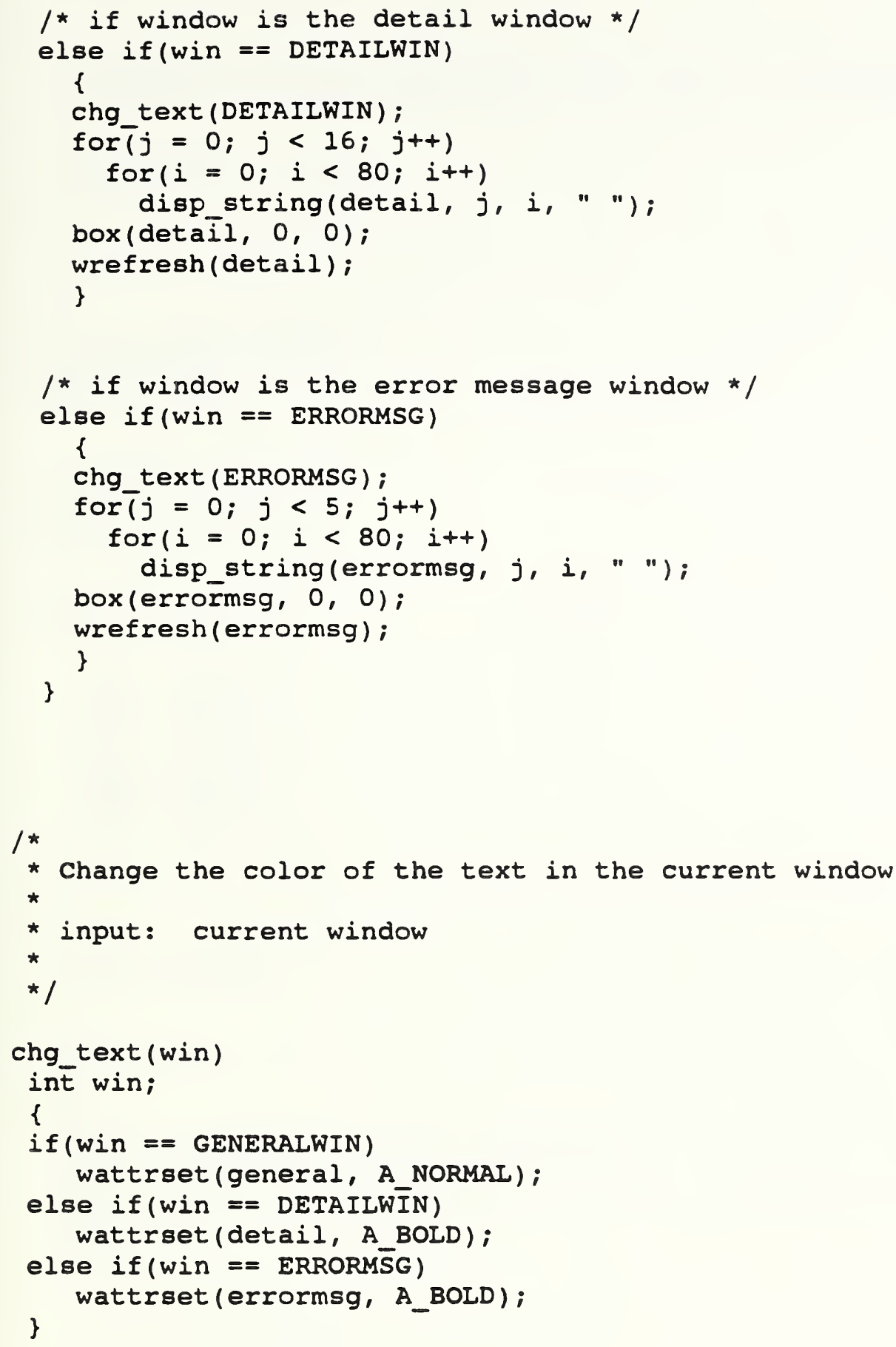





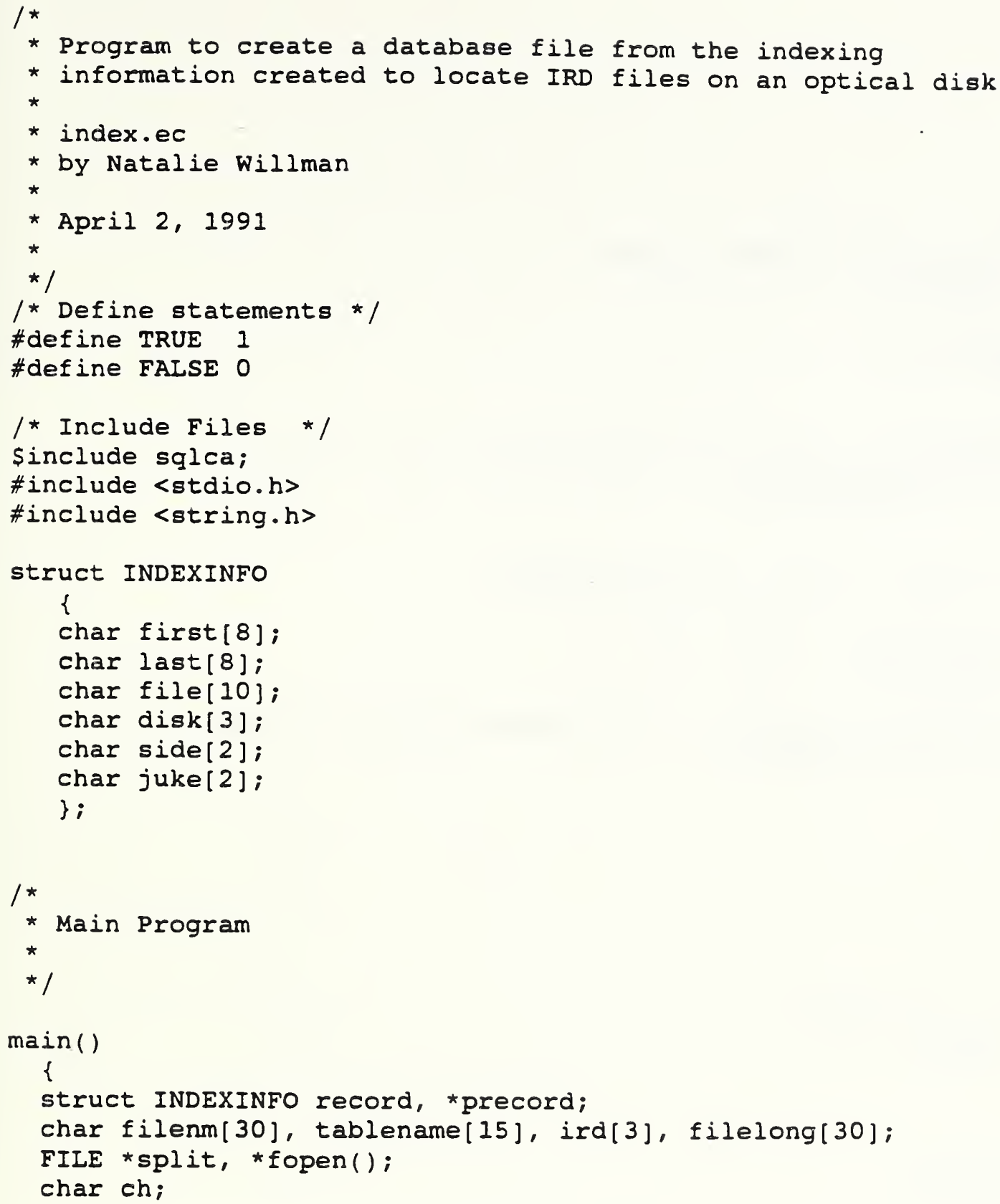


exit():

\}

\} 



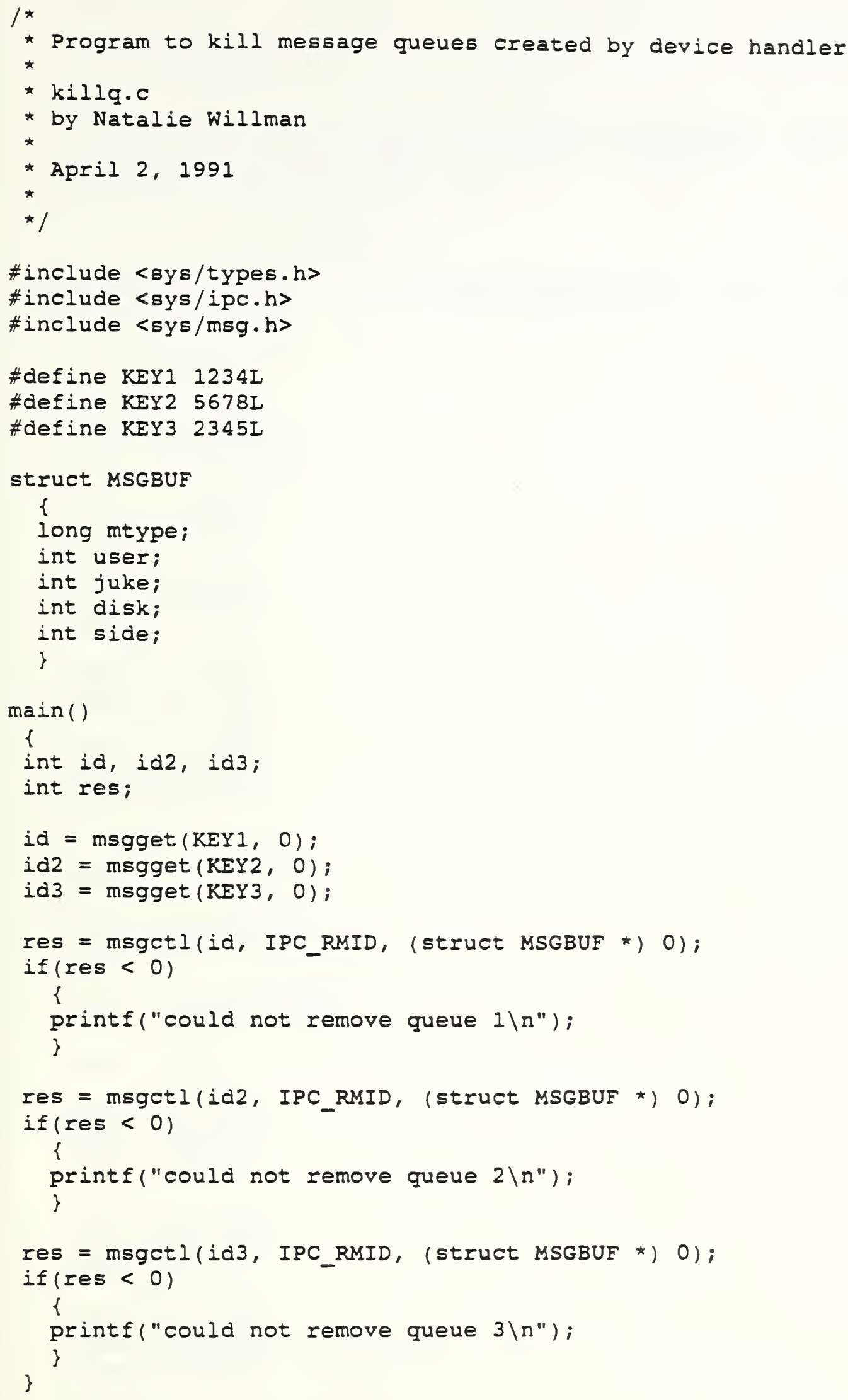





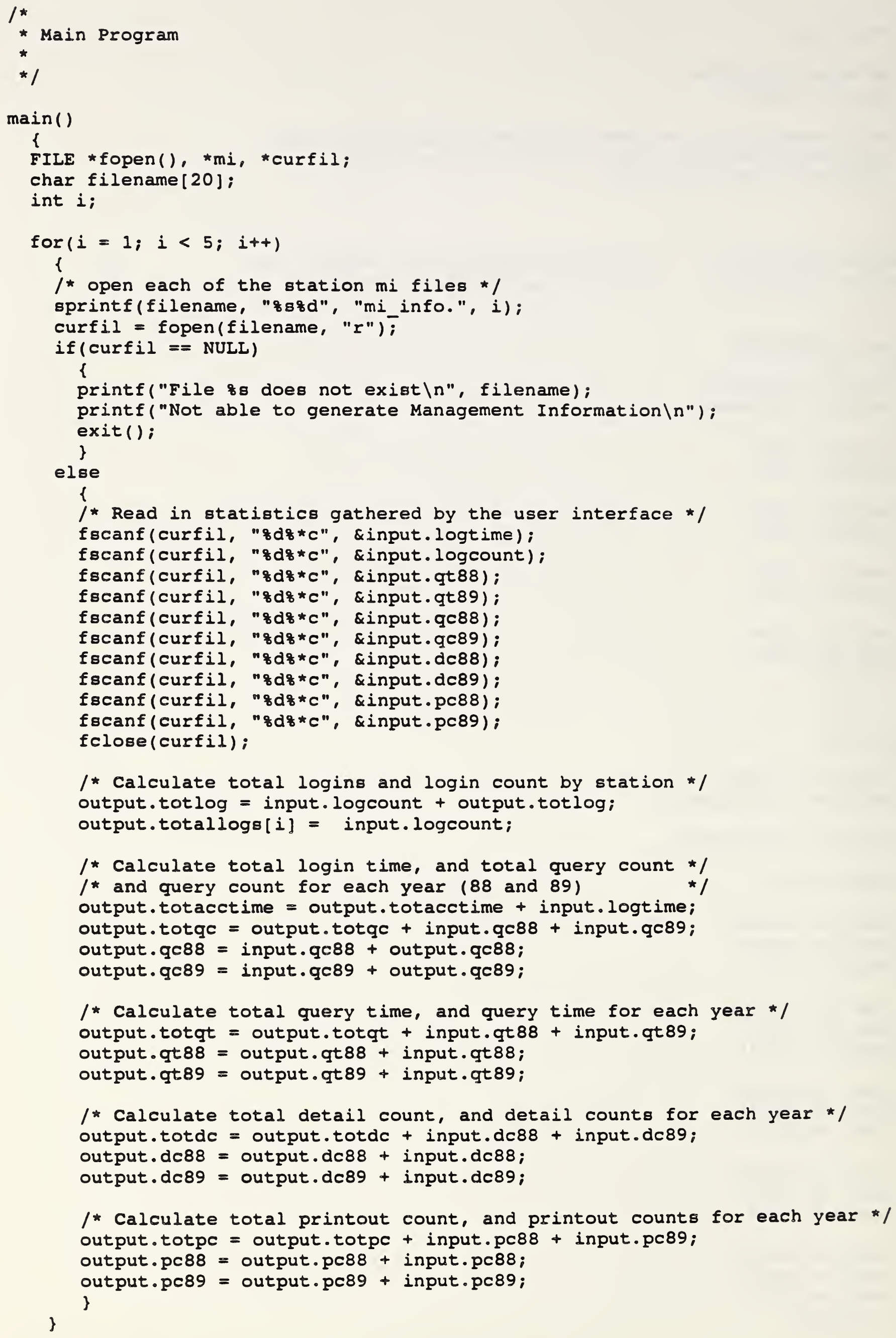




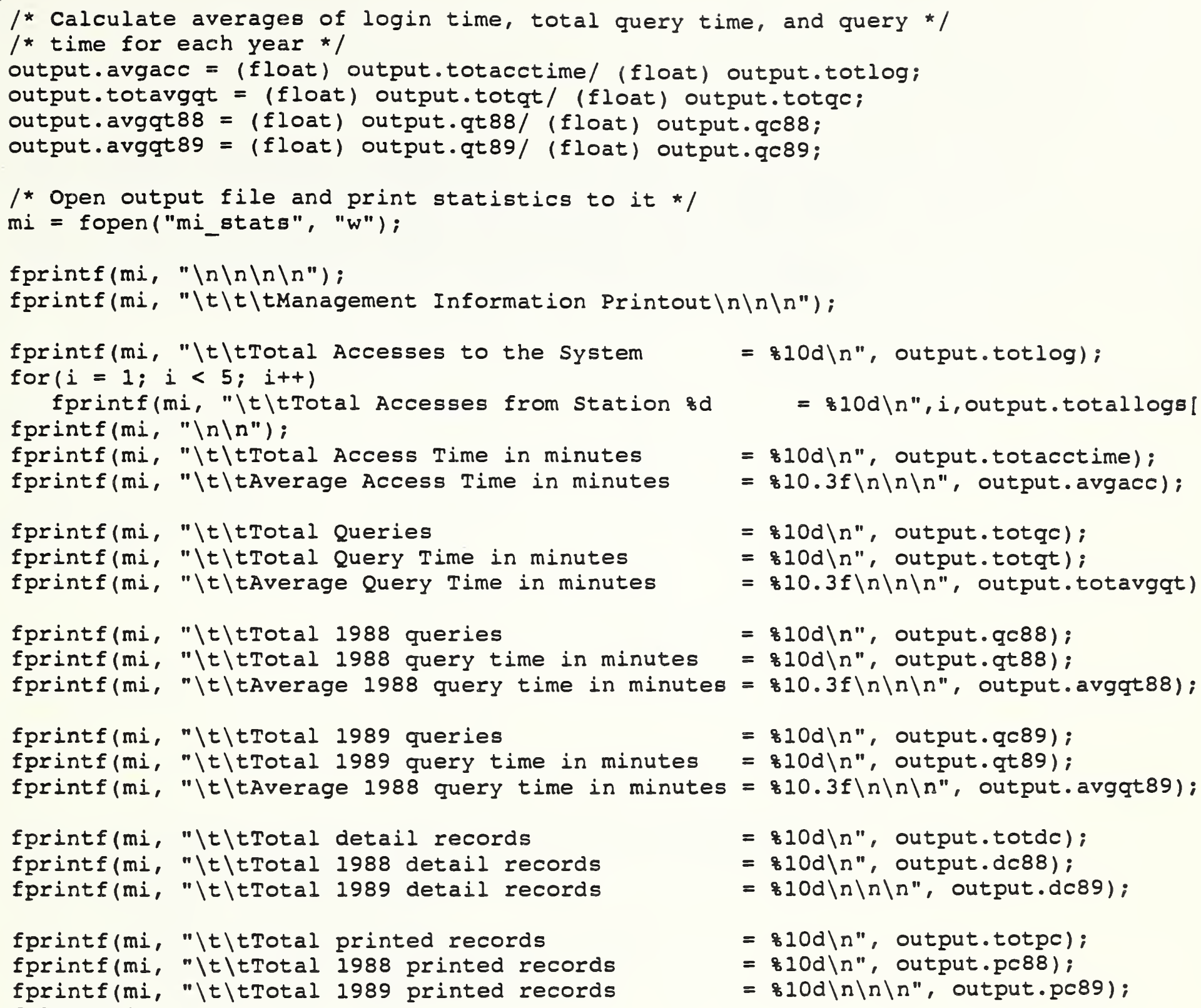

fprintf(mi, "\t\tAverage 1988 query time in minutes $=810.3 f \backslash n \backslash n \backslash n "$, output.avgqt 88 );

fprintf(mi, "\t\tAverage 1988 query time in minutes $=810.3 f \backslash n \backslash n \backslash n "$, output.avgqt89);

fprintf(mi, "\t|tTotal detail records fprintf(mi, "\t ttTotal 1988 detail records fprintf(mi, "\t\tTotal 1989 detail records

fprintf(mi, "\t\tTotal printed records fprintf(mi, "\t\tTotal 1988 printed records fprintf(mi, "\t\tTotal 1989 printed records

$=\& 10 d \backslash n ", i$, output.totallogs [

$=810 \mathrm{~d} \backslash \mathrm{n}$ ", output.totacctime):

$=810.3 f \backslash n \backslash n \backslash n "$, output.avgacc);

$=810 d \backslash n "$, output.totqc);

$=810 d \backslash n "$, output.totqt);

$=810.3 f \backslash n \backslash n \backslash n "$, output. totavgqt)

$=810 d \backslash n "$, output.qc88);

= $810 d \backslash n "$, output.qt88);

$=810 d \backslash n "$, output.totdc);

$=810 d \backslash \Omega^{\prime \prime}$, output.dc88);

$=810 d \backslash n \backslash \Omega \backslash \Omega$ ", output.dc89);

$=810 \mathrm{~d} \backslash \mathrm{n}$ ", output.totpc);

$=810 \mathrm{~d} \backslash \mathrm{n}^{\prime \prime}$, output.pc88);

$=810 \mathrm{~d} \backslash \mathrm{n} \backslash \mathrm{n} \backslash \mathrm{n}$ ", output.pc89); fclose (mi) ; \} 



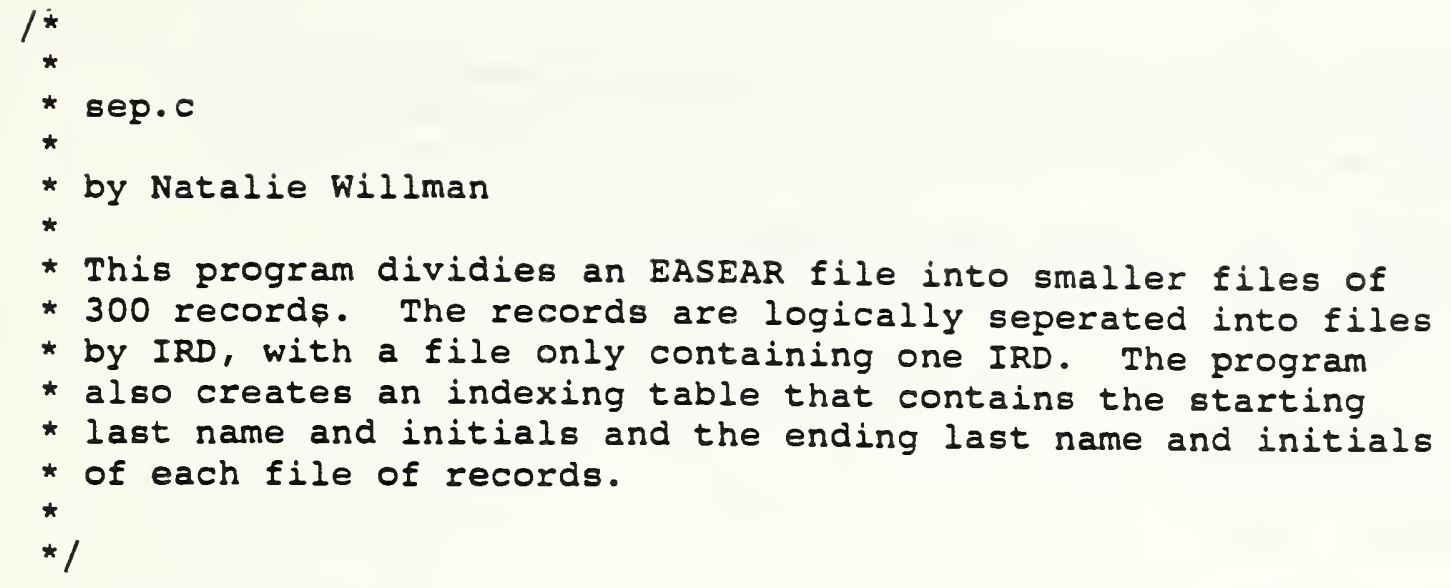

/* Include files */

\#include <stdio.h>

\#include <string.h>

/* Define statements */

* define tRUE 1

\#define FALSE 0

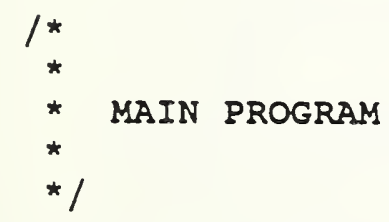

$\operatorname{main}()$

\{

int $i, j$, count, filenum, irdint, searchint, previrdint;

char filename[30], searchird[3];

char filenm[20], output[15], filestr[10];

char record $[235]$, errfile[20];

char ch, ird[3], last[10], init[4], previnit[4];

char prevlast[10], resp, namefile[20];

FILE *dat, *temp, *err, *name, *fopen();

int rec, reccount, end, filect;

int result, totalrec;

printf("Enter the IRD for which to search: $\mid n \backslash n ")$;

scanf ("zs\%*c", searchird);

printf $(" \backslash n ")$;

strcpy(resfile, "ird.");

strcat (resfile, searchird);

/* open the name file and print blank spaces for the initial last name */ sprintf(namefile, "zsss", "startend.", searchird);

name $=$ fopen (namefile, "w");

fprintf(name," $"$ );

fclose (name);

/* set up once only variables * /

reccount $=0$;

filect $=1$;

prevlast $[0]=0$;

previnit $[0]=0$; 
\{

printf("Make sure that the source disk is mounted as read-only $\backslash n$ "); printf("in Drive 2 and that the destination disk is mounted $\backslash n$ ");

printf("as read/write in drive $1 \backslash \mathrm{n} \backslash \mathrm{n}$ "):

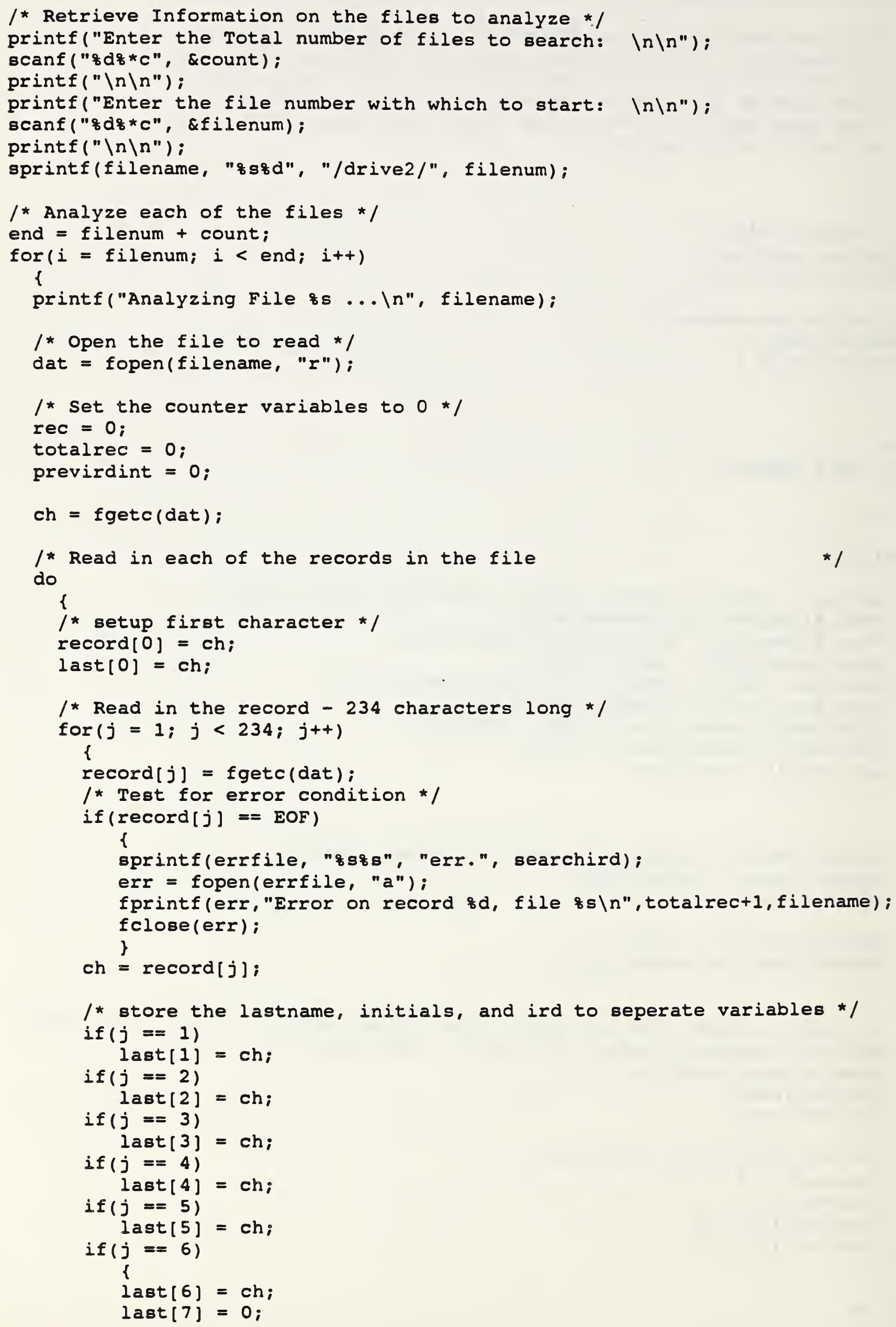




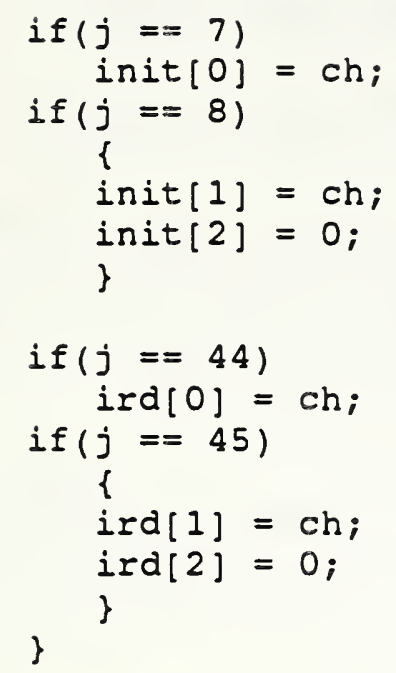




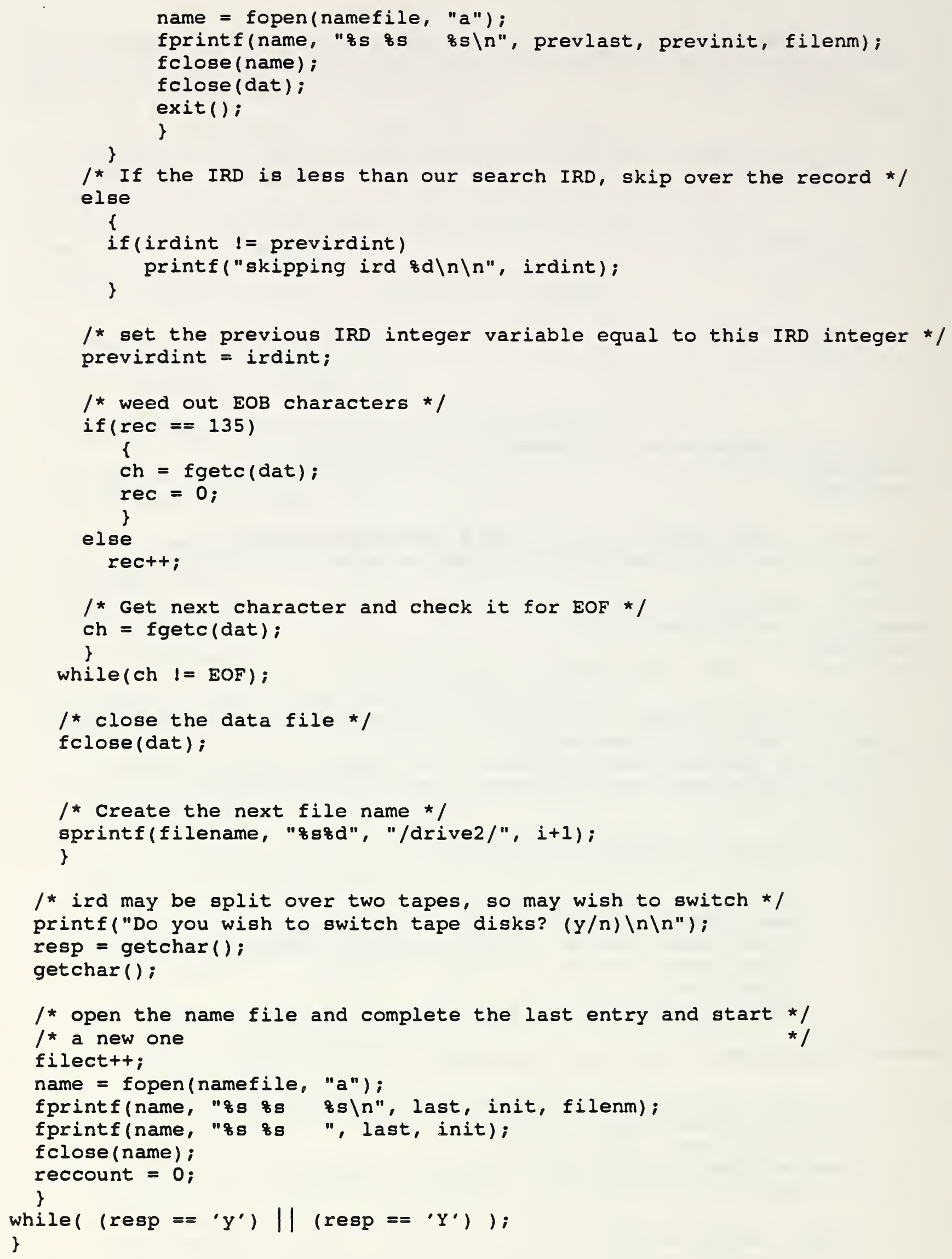




\begin{tabular}{|c|c|c|}
\hline \multirow[t]{3}{*}{$\begin{array}{l}\text { NIST-114A } \\
\text { (REV. 3-90) }\end{array}$} & \multirow{3}{*}{$\begin{array}{l}\text { U.S. DEPARTMENT OF COMMERCE } \\
\text { NATIONAL INSTITUTE OF STANDARDS AND TECHNOLOGY } \\
\text { BIBLIOGRAPHIC DATA SHEET }\end{array}$} & $\begin{array}{l}\text { 1. PUBLCATION OR REPORT MUMBER } \\
\text { NISTIR } 4654 \\
\end{array}$ \\
\hline & & 2. PERFORMING OAQANIZATION REPORT MUMBER \\
\hline & & $\begin{array}{l}\text { 3. PUBUCATION DATE } \\
\text { AUGUST } 1991\end{array}$ \\
\hline
\end{tabular}

Development of an Optical Disk System for the Automated Retrieval of EASEAR Records

5. AUTMOR(S)

Natalie Willman

6. PERFORMING ORGAMIZATION (IF JOINT OR OTHER THAN MIST, SEE INSTRUCTIONS)

U.S. DEPARTMENT OF COMMERCE

MATIONAL INSTITUTE OF STANDARDS AND TECHMOLOQY

CATTHERSBURQ, MO 20890

9. SPONSORING ORGANIZATION MAME AND COMPLETE ADDRESS (STREET, CITY, STATE, ZIP)

Social Security Administration

6401 Security Boulevard

Baltimore, MD 21235

\section{SUPPLEMENTARY NOTES}

11. ABSTRACT (A 200-WOAD OR LESS FACTUAL SUMMAAY OF MOST SIOMIFICANT IMFORMATION. BF DOCUMENT IMCLUDES A SIOMIFICANT BIBLOOQRAPHY OA UTERATURE SUAVEY, MENTION TT MERE,

The Social Security Administration (SSA) maintains records of the yearly wages earned by every person in the United States. Each year, approximately 2.5 gigabytes of data are collected on self-employed wage earners, and 47 gigabytes of data are collected on other wage earners. The records are currently stored on over 110,000 rolls of microfilm. It takes over 400 scouts to retrieve information needed by the professional staff. Often, the needed roll of film is missing from the file due to being currently in use, misfiled, or misplaced. In addition, the information obtained from the microfilm is not always the most current, and decisions are sometimes based on obsolete data. Advances in peripheral mass storage technology during the 1980 s (e.g. magneto-optic recording) now allow for alternate approaches to data storage and retrieval. It is believed that an automated retrieval system would provide a more accurate, timely, and cost effective means of retrieving information.

The pilot application detailed in this document is intended to demonstrate the feasibility of a full scale automated records retrieval system, to study the impact of an automated system on the users, and to gain insight into the factors which would impact the design of a full scale system.

\section{KEY WORDS (6 TO 12 ENTAIES; ALPHABETICN ORDER; CAPTTALE ONLY PROPEA MAMES; AND SEPARATE KEY WOADS EY SEMICOLONS)}

automated retrieval system; magneto-optic media; optical disk jukebox; optical disk media; pilot system

\section{AVALABILTYY}

UNUMUTED

FOR OFFICLLL DISTRIBUTION. DO NOT REESASE TO MATIOMAL TECHNICAL MFORMATION SERVICE (MTIS).

ORDER FROM SUPERINTENDENT OF DOCUMENTS, U.S. OOVERMMEMT PRINTING OFFICE, WASHINATON, DC 20402

ORDER FROM MATIOMAL TECHMICAL IMFORMATION SERVICE (WTIS), SPAIMOFIED, VA 22161.

14. MUMBER OF PRINTED PAGESS

147

15. PRICE

A07 


